\title{
The Hot Gas Exhaust of Starburst Engines in Mergers: Testing Models of Stellar Feedback and Star Formation Regulation
}

\author{
Beverly J. Smith \\ Department of Physics and Astronomy, East Tennessee State University, Johnson City TN 37614, USA \\ Peter Wagstaff \\ Department of Physics and Astronomy, East Tennessee State University Johnson City TN 37614, USA \\ Curtis Struck \\ Department of Physics and Astronomy, Iowa State University, Ames IA 50011, USA \\ Roberto Soria \\ College of Astronomy and Space Sciences, University of the Chinese Academy of Sciences, Beijing 100049, \\ China; Sydney Institute for Astronomy, School of Physics A28, The University of Sydney, Sydney, NSW \\ 2006, Australia \\ Brianne Dunn \\ Department of Physics and Astronomy, East Tennessee State University, Johnson City TN 37614, USA; \\ Now at Department of Physics and Astronomy, Clemson University, Clemson South Carolina 29634 \\ Douglas Swartz \\ Astrophysics Office, NASA Marshall Space Flight Center, ZP12, Huntsville AL 35812, USA \\ Mark L. Giroux \\ Department of Physics and Astronomy, East Tennessee State University Johnson City TN 37614, USA
}

\begin{abstract}
Using archival data from the Chandra X-ray telescope, we have measured the spatial extent of the hot interstellar gas in a sample of 49 nearby interacting galaxy pairs, mergers, and merger remnants. For systems with SFR $>1 \mathrm{M}_{\odot} \mathrm{yr}^{-1}$, the volume and mass of hot gas are strongly and linearly correlated with the star formation rate (SFR). This supports the idea that stellar/supernovae feedback dominates the production of hot gas in these galaxies. We compared the mass of X-ray-emitting hot gas $\mathrm{M}_{\mathrm{X}}$ (gas) with the molecular and atomic hydrogen interstellar gas masses in these galaxies $\left(\mathrm{M}_{\mathrm{H}_{2}}\right.$ and $\mathrm{M}_{\mathrm{HI}}$, respectively), using published carbon monoxide and $21 \mathrm{~cm} \mathrm{HI}$ measurements. Systems with higher SFRs have larger $\mathrm{M}_{\mathrm{X}}$ (gas)/( $\left.\mathrm{M}_{\mathrm{H}_{2}}+\mathrm{M}_{\mathrm{HI}}\right)$ ratios on average, in agreement with recent numerical simulations of star formation and feedback in merging galaxies. The $\mathrm{M}_{\mathrm{X}}(\mathrm{gas}) /\left(\mathrm{M}_{\mathrm{H}_{2}}+\mathrm{M}_{\mathrm{HI}}\right)$ ratio also increases with dust temperature on average. The ratio $\mathrm{M}_{\mathrm{X}}$ (gas)/SFR is anti-correlated with the IRAS $60 \mu \mathrm{m}$ to $100 \mu \mathrm{m}$ flux ratio and with the Spitzer $3.6 \mu \mathrm{m}$ to $24 \mu \mathrm{m}$ color. These trends may be due to variations in the spatial density of young stars, the stellar age, the ratiol of young to old stars, the initial mass function, and/or the efficiency of stellar feedback. Galaxies with low SFR $\left(<1 \mathrm{M}_{\odot} \mathrm{yr}^{-1}\right)$ and high $\mathrm{K}$ band luminosities may have an excess of hot gas relative to the relation for higher SFR galaxies, while galaxies with low $\mathrm{K}$ band luminosities (and therefore low stellar masses) may have a deficiency in hot gas, but our sample is not large enough for strong statistical significance.
\end{abstract}




\section{Introduction}

Feedback from stellar winds, radiation pressure, and supernovae play a major role in regulating star formation, by heating, ionizing, and accelerating the interstellar gas and adding turbulence. However, the details of these processes are not well-understood. Computer simulations are frequently used to study stellar feedback and star formation, using various prescriptions to model the feedback. These processes are complicated to model because different feedback mechanisms help regulate star formation in different ways, multiple mechanisms operate simultaneously, and the different mechanisms affect each other. Radiation pressure from young stars disrupts molecular clouds, decreasing the amount of dense gas and preventing too-rapid gravitational collapse of clouds (Hopkins et al. 2011, 2013b, 2014), while shock heating by supernovae and stellar winds is responsible for most of the hot X-ray-emitting gas in galaxies (Hopkins et al. 2012b). Before supernovae begin in a young star-forming region, radiation pressure and stellar winds clear out dense gas in star forming regions, heating and stirring the gas; later supernovae thus occur in lower density gas, causing the hot gas to survive longer and inhibiting subsequent star formation Hopkins et al. 2012a; Agertz et al. 2013). The more efficient the feedback, the lower the efficiency of subsequent star formation (Cox et al. 2006c; Hopkins et al. 2013b). Supernovae provide both thermal energy, heating the gas, as well as kinetic feedback, which increases turbulence and thus affects later star formation (Springel 2000; Hopkins et al. 2014). Another way feedback regulates star formation is by removing gas from the main disk of the galaxy, either temporarily or permanently (e.g., Muratov et al. 2015). Supernova-driven winds may drive gas out into the halo; this hot halo material may then cool and fall back in on the galaxy, triggering delayed star formation Hopkins et al. 2013). Winds due to supernovae may remove gas from the galaxy entirely; in some simulations the mass loss rate from supernovae-driven winds is greater than the star formation rate (SFR) (Hopkins et al. 2012a, 2013).

The latest generation of simulations include multi-phase interstellar gas, to follow both the dense cores of molecular clouds where star forma- tion occurs, the warmer atomic gas, and the hot intracloud medium (Hopkins et al. 2013b, 2014; Renaud et al. 2013, 2014, 2015, 2019; Sparre \& Springel 2016a; Fensch et al. 2017; (Moreno et al. 2019). The results of such simulations sometimes depend upon the resolution of the simulation and the details of the calculations, with higher resolution models producing more efficient star formation (Teyssier, Chapon, \& Bournaud 2010; Hopkins et al. 2013; Hayward et al. 2014; Sparre \& Springel 2016a), and the duration and intensity of a starburst depending upon the prescription for feedback assumed in the model (Hopkins et al. 2012a; Fensch et al. 2017). How stellar feedback is implemented in these codes has profound cosmological consequences. Stellar feedback is needed in cosmological simulations of galaxy formation and evolution to explain the observed galaxy mass function (Kereš et al. 2009), the galaxy stellar mass-halo mass relation (Hopkins et al. 2014; Agertz \& Kravtsov 2015, 2016; Trujillo-Gomez et al. 2015) and the galaxian mass-metallicity relation (Finlator \& Davé 2008; Ma et al. 2016; Torrev et al. 2019). For cosmological models to reproduce the so-called galaxy main sequence (a correlation between stellar mass and star formation; Brinchmann et al. 2004; Noeske et al. 2007; Salim et al. 2007) or the Kennicutt-Schmidt Law (a relation between SFR and molecular gas mass; Schmidt 1959; Kennicutt 1998; Kennicutt \& Evans 2012), stellar feedback is necessary (Hopkins et al. 2014; Orr et al. 2018).

To test these feedback models, X-ray observations are required. With high resolution X-ray imaging, the distribution, temperature, and mass of the hot gas within galaxies can be studied, and compared to other properties of the galaxies. In star-forming galaxies, the bulk of the hot gas is attributed to feedback from Type II supernova and young stars (Strickland et al. 2000, 2004a,b; Grimes et al. 2005; Owen \& Warwick 2009; Li \& Wang 2013; Mineo et al. 2012b; Smith et al. 2018). Hopkins et al. (2012a) model the X-ray production due to stellar feedback in different types of galaxies, and conclude that for normal spirals and dwarf galaxies, supernovae and stellar winds dominate, but in intense starbursts radiation pressure dominates. The soft X-rays from galactic winds originate from a small fraction of the total hot gas; the bulk of the hot 
gas is such low density it is difficult to observe directly (Strickland \& Stevens 2000). Freely-flowing hot gas produces little X-ray emission, in contrast to hot gas confined by surrounding cooler gas (Hopkins et al. 2012a). Observational studies show that for star-forming galaxies, the X-ray luminosity from hot gas, $\mathrm{L}_{\mathrm{X}}$ (gas), is proportional to the SFR (Strickland et al. 2004b; Grimes et al. 2005; Mineo et al. 2012b; Smith et al. 2018). This is in contrast to some theoretical estimates, which predict that $\mathrm{L}_{\mathrm{X}}$ (gas) should be proportional to $\mathrm{SFR}^{2}$ (Chevalier \& Clegg 1985; Zhang et al. 2014). More modern theoretical calculations including gravitational forces and improved radiative cooling are able to reproduce the observed $\mathrm{L}_{\mathrm{X}}($ gas $) \propto \mathrm{SFR}$ relation for star-forming galaxies if the mass-loading factor (mass outflow rate/SFR) decreases as SFR increases (Bustard, Zweibel, \& D'Onghia 2016; Meiksin 2016). The recent cosmological hydrodynamical simulations of van de Voort et al. (2016) including feedback find a constant $\mathrm{L}_{\mathrm{X}}$ (gas)/SFR ratio for galaxies with halo masses between $10^{10.5}$ $-10^{12} \mathrm{M}_{\odot}$, where the Milky Way has a halo mass of $\sim 10^{12} \mathrm{M}_{\odot}$.

Over timescales of many gigayears, virialization of gas provided by stellar mass loss from older stars can contribute to the X-ray-emitting hot gas in galaxies, particularly in massive galaxies with low SFRs (e.g., Ciotti et al. 1991; Pellegrini \& Ciotti 1998; Mathews \& Brighenti 2003). In quiescent early-type galaxies, this contribution dominates, as $\mathrm{L}_{\mathrm{X}}$ (gas) increases with mass rather than with SFR (e.g., O'Sullivan et al. 2001a; Kim \& Fabbiano 2013; Su et al. 2015; Goulding et al. 2016). The possible existence of this additional source of hot gas may need to be taken into account in interpreting X-ray data in terms of stellar feedback, particularly in galaxies with low SFRs and high masses.

In the current study, our goal is to track the evolution of the hot gas in galaxies compared to the other components of the galaxies, particularly the molecular and atomic gas, and compare with expectations from theoretical models. This study is a follow-up to our earlier archival Chandra study of 49 nearby major mergers in a range of merger stages (Smith et al. 2018, hereafter Paper I). In the earlier study, we removed the resolved point sources and extracted the spectrum of the dif- fuse X-ray emission. We then separated this spectrum into a power law and a thermal component and corrected for internal absorption. Assuming the thermal component was due to hot gas, we compared the thermal luminosity $\mathrm{L}_{\mathrm{X}}$ (gas) with the global SFR as derived from UV/optical data. Although there is considerable system-to-system variation in the $\mathrm{L}_{\mathrm{X}}$ (gas)/SFR ratio, we did not see any trends of $\mathrm{L}_{\mathrm{X}}$ (gas)/SFR with merger stage, active galactic nuclei (AGN) activity, or SFR for galaxies with $\mathrm{SFR}>1 \mathrm{M}_{\odot} \mathrm{yr}^{-1}$. These results suggest that in star-forming galaxies, stellar feedback reaches an approximately steady-stage condition. In Paper I, we concluded that, for star forming galaxies, about $2 \%$ of the total energy output from supernovae and stellar winds is converted into X-ray flux; this result is in agreement with earlier results from smaller samples of galaxies (Grimes et al. 2005; Mineo et al. 2012b).

In the current study, we revisit the same sample of mergers, and use the Chandra data to derive the spatial extent of the hot gas in these galaxies and therefore the mass of hot X-ray-emitting gas $\mathrm{M}_{\mathrm{X}}$ (gas). We compare $\mathrm{M}_{\mathrm{X}}$ (gas) with the amount of cold molecular and atomic hydrogen gas in these galaxies, as obtained from published carbon monoxide and $21 \mathrm{~cm} \mathrm{HI}$ observations. Our goal is to better understand how interstellar gas cycles between hot and cold phases due to star formation and stellar feedback, and how this cycle affects the efficiency of star formation (SFE).

In Section 2 of this paper, we review the selection of the sample and the available ultraviolet, infrared, and optical data. In Section 3, we explain the molecular and atomic hydrogen gas data. In Section 4, we determine the spatial extent of the hot gas in the galaxies. We obtain the volume and mass of hot X-ray-emitting gas and the electron density in Section 5. These values are then compared with other parameters of the systems in Section 6. The results are discussed in Section 7, and conclusions are provided in Section 8.

\section{Sample Selection and UV/IR/Optical Data}

The sample selection is described in detail in Paper I. Briefly, the sample includes 49 pre-merger interacting pairs, post-merger remnants, and midmerger systems in the nearby Universe (distance 
$<180 \mathrm{Mpc}$ ). Initially, galaxies were chosen based on their morphologies from the Arp (1966) Atlas of Peculiar Galaxies, or from other published surveys of mergers and merger remnants, selecting approximately equal-mass interacting pairs or the remnants of the merger of such pairs. The final sample was then selected based on the availability of suitable Chandra data. See Paper I for details.

The sample of galaxies is given in Table 1. Table 1 also provides basic data on these systems from Paper I, including distances assuming a Hubble constant of $73 \mathrm{~km} \mathrm{~s}^{-1} \mathrm{Mpc}^{-1}$, correcting for peculiar velocities due to the Virgo Cluster, the Great Attractor, and the Shapley Supercluster. The median distance for our sample galaxies is $51.5 \mathrm{Mpc}$. Table 1 also provides the far-infrared luminosity $\mathrm{L}_{\mathrm{FIR}}$ and the near-infrared $\mathrm{K}$ band luminosity $\mathrm{L}_{\mathrm{K}}$, obtained from Infrared Astronomical Satellite (IRAS) and 2-micron All-Sky Survey (2MASS) data, respectively, as described in Paper I. In addition, Table 1 includes SFRs, derived from a combination of Spitzer infrared and GALEX UV photometry as described in Paper I. When available, the far-UV (FUV) is used; otherwise, near-UV (NUV) photometry is used. These SFRs correspond to the SFR averaged over a time period of 100 Myrs (Kennicutt \& Evans 2012). Table 1 also identifies the 13 galaxies in the sample that are classified in the NASA Extragalactic Database (NED11) as Seyfert 1, Seyfert 2, or Low Ionization Nuclear Emission Region (Liner) galaxies. Detailed descriptions of the individual galaxies in the sample are provided in the Appendix of Paper I.

Based on their morphologies, in Paper I we classified the systems into seven merger stages. These stages are: (1) separated but interacting pair with small or no tails, (2) separated pair with moderate or long tails, (3) pair with disks in contact, (4) common envelope, two nuclei, and tails, (5) single nucleus and two strong tails, (6), single nucleus but weak tails, and (7) disturbed elliptical with little or no tails. The staging is approximate, with an uncertainty of \pm 1 stage.

In Figure 1, various properties of the galaxies (distance, $\mathrm{L}_{\mathrm{FIR}}$, the $\mathrm{L}_{\mathrm{FIR}} / \mathrm{L}_{\mathrm{K}}$ ratio, and the IRAS $60 \mu \mathrm{m}$ to $100 \mu \mathrm{m}$ flux ratio $\left.\mathrm{F}_{60} / \mathrm{F}_{100}\right)$ are plotted against the merger stage. The black open squares

\footnotetext{
${ }^{1}$ http://ned.ipac.caltech.edu
}

in Figure 1 are the data for the individual galaxies; the blue filled diamonds that are offset slightly to the left of the stage show the median value for that stage. The errorbars on the blue diamonds show the semi-interquartile range, equal to half the difference between the 75 th percentile and the 25th percentile. As discussed in Paper I, this sample is inhomogeneous because it was selected based on the availability of archival Chandra data. As illustrated in Figure 1, the sample has some biases. The galaxies in the middle of the merger sequence tend to be more distant and so tend to have higher FIR luminosities. This means they have higher SFRs, since $\mathrm{L}_{\mathrm{FIR}}$ is an approximate measure of the SFR for galaxies with high SFRs (e.g., Kennicutt 1998; Kennicutt \& Evans 2012). The late-stage mergers tend to be closer and have lower $\mathrm{L}_{\mathrm{FIR}}$. Late-stage mergers are difficult to identify at large distances, thus confirmed examples tend to be nearby.

The late-stage mergers also tend to have lower $\mathrm{L}_{\mathrm{FIR}} / \mathrm{L}_{\mathrm{K}}$ ratios. This ratio is an approximate measure of the specific SFR (sSFR), defined as the SFR/stellar mass, since the $\mathrm{K}$ band luminosity $\mathrm{L}_{\mathrm{K}}$ is an approximate measure of the stellar mass (Maraston 1998; Bell \& de Jong 2000; Into \& Portinari 2013; Andreani et al. 2018), although it is affected by age and possible AGN contributions. The mid-merger systems also tend to have higher dust temperatures, as traced by the IRAS $\mathrm{F}_{60} / \mathrm{F}_{100}$ ratio (last panel Figure 1). The uncertainty in the staging, the biases in the sample, and the small number of systems in each stage means trends with merger stage are uncertain. As seen in Figure 1, the AGN tend to be mid-merger systems with high $\mathrm{L}_{\mathrm{FIR}}$ and $\mathrm{F}_{60} / \mathrm{F}_{100}$. Although AGN can contribute to the heating of interstellar dust in galaxies, for most of our AGNs published studies of the IR spectra of the galaxies conclude that dust heating is dominated by star formation rather than the AGN (see the detailed discussions on the individual galaxies in the Appendix of $\mathrm{Pa}$ per I).

Figure 2 displays some well-known correlations between these basic parameters. The observed correlation between SFR and $\mathrm{L}_{\mathrm{K}}$ (top left panel) or its equivalent has been seen many times before for star forming galaxies (e.g., Smith et al. 1998; Andreani et al. 2018). This relation is a consequence of the correlation between SFR 
and stellar mass, which is known as the 'galaxy main sequence' for star forming galaxies (e.g., Brinchmann et al. 2004; Salim et al. 2007). For our sample, this correlation is only a weak correlation, because of the inclusion of some systems with low SFRs compared to $\mathrm{L}_{\mathrm{K}}$. Galaxies with low SFR compared to the best-fit 'galaxy main sequence' relation are considered quenched, quenching, or post-starburst galaxies. In our sample, our post-starburst galaxies are all late-stage mergers, and have low $\mathrm{L}_{\mathrm{FIR}} / \mathrm{L}_{\mathrm{K}}$ ratios.

Figure 2 shows that the SFR is correlated with both $\mathrm{L}_{\mathrm{FIR}} / \mathrm{L}_{\mathrm{K}}$ and the Spitzer [3.6 $\left.\mu \mathrm{m}\right]-[24 \mu \mathrm{m}]$ color for our sample galaxies. 2. The majority of our galaxies fall in a narrow range of $\mathrm{L}_{\mathrm{FIR}} / \mathrm{L}_{\mathrm{K}}$, $-1 \leq \log \mathrm{L}_{\mathrm{FIR}} / \mathrm{L}_{\mathrm{K}} \leq 0$, but a handful have lower $\mathrm{L}_{\mathrm{FIR}} / \mathrm{L}_{\mathrm{K}}$ ratios (the post-starburst systems with low SFRs) and a few have higher $\mathrm{L}_{\mathrm{FIR}} / \mathrm{L}_{\mathrm{K}}$ ratios. The [3.6] - [24] color is an approximate measure of the ratio of the number of young-to-old stars (e.g., Smith et al. 2007), increasing with increasing proportions of young stars. This means that $[3.6]-[24]$ is another approximate measure of the sSFR.

Figure 2 also shows that $\mathrm{F}_{60} / \mathrm{F}_{100}$ is weakly correlated with SFR, with considerable scatter. This relation or its equivalent has been noted before (e.g., Soifer et al. 1987; Smith et al. 1987). Higher $\mathrm{F}_{60} / \mathrm{F}_{100}$ ratios imply hotter dust on average and more intense UV interstellar radiation fields (ISRF) (e.g., Desert. Boulanger. \& Puget 1990), which are correlated but not perfectly with the overall SFR of the galaxy.

\section{Atomic and Molecular Interstellar Gas}

In the current study, we compare the hot Xray-emitting gas mass in these galaxies with the interstellar molecular and atomic hydrogen gas masses. We obtained published measurements of the $2.6 \mathrm{~mm} \mathrm{CO}(1-0)$ fluxes of the sample galaxies from the literature, and used these to derive molecular gas masses. Since there is some uncertainty as to the relation between the CO luminosity and the molecular gas mass, we converted the $\mathrm{CO}$ fluxes into molecular gas masses $\mathrm{M}_{\mathrm{H}_{2}}$ by two methods. First, we calcu-

\footnotetext{
$2[3.6]-[24]$ is defined as the magnitude in the $3.6 \mu \mathrm{m}$ filter minus that in the $24 \mu \mathrm{m}$ filter, using zero magnitude flux densities of $277.5 \mathrm{Jy}$ and $7.3 \mathrm{Jy}$, respectively.
}

lated $\mathrm{M}_{\mathrm{H}_{2}}$ for all galaxies assuming a constant conversion equal to the Galactic conversion factor between $\mathrm{H}_{2}$ column density $\mathrm{N}\left(\mathrm{H}_{2}\right)\left(\mathrm{cm}^{-2}\right)$ and $\mathrm{CO}$ intensity $\mathrm{I}(\mathrm{CO})$ of $\mathrm{N}\left(\mathrm{H}_{2}\right)\left(\mathrm{cm}^{-2}\right)=2.0$ $\times 10^{20} \mathrm{I}(\mathrm{CO})\left(\mathrm{K} \mathrm{km} \mathrm{s}^{-1}\right)$ (Dame et al. 2001; Bolatto, Wolfire, \& Leroy 2013). The Galactic conversion is thought to be appropriate for most galaxies, however, low metallicity systems may be deficient in $\mathrm{CO}$ relative to $\mathrm{H}_{2}$, while extreme starburst galaxies may have enhanced $\mathrm{CO} / \mathrm{H}_{2}$ ratios (e.g., Downes \& Solomon 1998; Bolatto, Wolfire, \& Leroy 2013). Thus for comparison we made a second estimate of $\mathrm{M}_{\mathrm{H}_{2}}$ using a variable $\mathrm{CO} / \mathrm{H}_{2}$ ratio. For galaxies with $\mathrm{L}_{\mathrm{FIR}}>$ $10^{11} \mathrm{~L}_{\odot}$ (e.g., extreme starbursts), we used a lower conversion factor of $4 \times 10^{19} \mathrm{~cm}^{-2} /\left(\mathrm{K} \mathrm{km} \mathrm{s}^{-1}\right)$ (e.g., Ueda et al. 2014). For galaxies with low $\mathrm{K}$ band luminosities (e.g., possible low metallicity systems), $\mathrm{L}_{\mathrm{K}}<10^{10} \mathrm{~L}_{\odot}$, we used an enhanced ratio of $5 \times 10^{20} \mathrm{~cm}^{-2} /\left(\mathrm{K} \mathrm{km} \mathrm{s}^{-1}\right)$ (e.g., Bolatto. Wolfire, \& Leroy 2013). For all other galaxies we used the standard Galactic value given above. Since accurate metallicities are not available for all of the galaxies in our sample and because there is some uncertainty as to how the $\mathrm{CO} / \mathrm{H}_{2}$ ratio varies with metallicity, we do not use a more complicated metallicity-dependent conversion in this study. In Section 6 of this paper, we compare various properties of the galaxies. We do the correlation analysis with both $\mathrm{CO} / \mathrm{H}_{2}$ ratios, to test whether our conclusions are influenced by our choice of $\mathrm{CO} / \mathrm{H}_{2}$ conversion factors.

Molecular gas masses calculated with a constant $\mathrm{CO} / \mathrm{H}_{2}$ ratio equal to the Galactic value are provided in column 2 of Table 2. Molecular gas masses calculated with the variable $\mathrm{CO} / \mathrm{H}_{2}$ ratio are given in column 3 of Table 2. The reference for the original $\mathrm{CO}$ measurement is given in column 4 of Table 2. Note that molecular masses are not available for all of the galaxies in the sample. In some cases, no CO observations have been published. In other cases, only measurements of the central region have been made, where the beamsize is significantly smaller than the optical extent of the galaxy. In those cases, we are not able to get reliable upper limits to the global molecular gas content so no molecular gas mass is listed in Table 2. Followup CO observations would be useful to complete the molecular gas census of the sample galaxies. 
In the bottom row of Figure 3, the star formation efficiency, which we define as the global $\mathrm{SFR} / \mathrm{M}_{\mathrm{H}_{2}}$ ratio for the galaxy 3 , is plotted against the merger stage. The left panel of Figure 3 has SFE calculated with a constant $\mathrm{CO} / \mathrm{H}_{2}$ ratio and the right with the variable $\mathrm{CO} / \mathrm{H}_{2}$ ratio. These two determinations of the SFE are included in Table 2 in columns 5 and 6 , respectively. As in Figure 1, the black open squares in Figure 3 are the data for the individual galaxies; the blue filled diamonds that are offset slightly to the left of the stage show the median value for that stage. The errorbars on the blue diamonds show the semiinterquartile range, equal to half the difference between the 75 th percentile and the 25 th percentile.

Systems in the middle merger stages tend to have higher SFEs than those in the early stages. This is consistent with earlier surveys that found that $\mathrm{L}_{\mathrm{FIR}} / \mathrm{M}_{\mathrm{H}}$, is enhanced near nuclear coalescence (Casoli et al. 1991; Georgakakis et al. 2000). Given the small numbers of galaxies per merger stage in our sample and the spread in the data per merger stage, however, this result is uncertain for our sample, especially if one also takes into account the uncertainties in the $\mathrm{CO} / \mathrm{H}_{2}$ ratio, and the selection effects. Because of these factors any trends with merger stage are uncertain for our sample.

We also scoured the literature for measurements of the global HI masses of our galaxy sample. These values are tabulated in column 7 of Table 2, and the reference for the HI data is given in column 8. In Figure 3, we provide plots of merger stage vs. quantities derived from the $\mathrm{CO}$ and $\mathrm{HI}$ data. The top row of Figure 3 compares $\mathrm{M}_{\mathrm{HI}} / \mathrm{M}_{\mathrm{H}_{2}}$ with the merger stage. In the left panel, we assume a constant $\mathrm{CO} / \mathrm{H}_{2}$ ratio in calculating $\mathrm{M}_{\mathrm{H}_{2}}$, while in the right panel we use the variable $\mathrm{CO} / \mathrm{H}_{2}$ ratio. An apparent increase in the HI gas fraction in the late stages of the merger sequence (left panel) is weakened when a variable $\mathrm{CO} / \mathrm{H}_{2}$ ratio is used (right panel).

The SFE is plotted against dust temperature as measured by the IRAS $60 \mu \mathrm{m}$ to $100 \mu \mathrm{m}$ flux ratio in the two top panels of Figure 4, for the two $\mathrm{CO} / \mathrm{H}_{2}$ conversion factors. A trend is clearly

\footnotetext{
${ }^{3}$ With this definition, the SFE is equal to $1 / \tau_{\text {dep }}$, where $\tau_{\text {dep }}$ is the global depletion timescale, the time to use up the molecular gas.
}

visible, in that hotter dust is correlated with more efficient star formation. This relation is well-known (e.g., Young et al. 1986; Sanders et al. 1991). Note that the scatter is larger with the variable $\mathrm{CO} / \mathrm{H}_{2}$ ratio than for the constant conversion factor. In the bottom two panels of Figure 4, we compare the SFE with the SFR for the two conversion factors. There is a trend, in that systems with the highest SFRs have high SFEs, however, there is a lot of scatter, and there are some low SFR systems with high SFE. A spread in the SFE for a given SFR has been observed before (e.g., Young et al. 1986; Sanders et al. 1991; Young et al. 1996; Sanders \& Mirabel 1996; Daddi et al. 2010).

The scatter in the SFE vs. SFR correlation may be due to variations in the fraction of the CO-emitting gas involved in star formation. This would lead to variations in the SFE according to our definition, $\mathrm{SFR} / \mathrm{M}_{\mathrm{H}_{2}}$, where $\mathrm{H}_{2}$ is derived from $\mathrm{CO}$ observations. Larger SFE may mean that a larger fraction of the CO-emitting cold molecular gas is in a dense state, an idea that is supported by both observations (Solomon et al. 1992; Gao \& Solomon 2004; Juneau et al. 2009; Wu et al. 2010) and simulations (Tevssier. Chapon, \& Bournaud 2010; Renaud et al. 2014, 2019; Sparre \& Springel 2016a). These simulations show that an increase in turbulent compression during an interaction can cause the gas probability density function to shift to higher densities, producing an increase in the amount of very high density gas. Thus the variations in SFE from galaxy to galaxy may be caused by differences in the dynamical state of the galaxies.

\section{X-Ray Spatial Extent}

All of the sample galaxies were observed with the Chandra ACIS-S array, and all of the galaxies fit well within the $8.3 \times 8.3$ field of view of the $\mathrm{S} 3$ chip of this array. Details of the individual observations, including exposure times and ObsID numbers, are provided in Paper I. In Paper I, we identified point sources in the field using the ciao software tool wavdetect. The point sources themselves and their statistics were the subject of another paper (Smith et al. 2012).

After removing the point sources, in Paper I we 
used the Chandra Interactive Analysis of Observations (ciao) software routine specextract to extract the diffuse X-ray spectrum within the optical B band $25 \mathrm{mag} \operatorname{arcsec}^{-2}$ isophote. This optical isophote was measured on Sloan Digitized Sky Survey (SDSS) g images using standard g-toB conversion factors, or, if SDSS images weren't available, equivalent levels on GALEX near-UV images were used (see Smith et al. 2018 for details). In Paper I, we used the xsped software to fit the $0.3-8.0 \mathrm{keV}$ background-subtracted point-source-removed spectrum within the $\mu_{\mathrm{B}}=$ $25 \mathrm{mag} \operatorname{arcsec}^{-2}$ isophote (e.g., D25) to a combination power law plus thermal (MEKAL) spectrum, assuming a power law photon index of 1.8 and correcting for both Galactic and internal absorption. The power law component is assumed to be caused by faint unresolved point sources. The absorption-corrected $0.3-8.0 \mathrm{keV}$ luminosities for the MEKAL component are provided in Table 1 of the current paper; we assume that the MEKAL component is from hot gas. These $\mathrm{X}-$ ray luminosities have been corrected for absorption within the galaxies as described in Paper I.

In the current study, we measured the spatial extent of the diffuse soft X-ray flux in these galaxies, and use these estimates to calculate the electron densities and masses of the hot X-rayemitting gas. Our procedure is as follows. After initial processing and deflaring of the data as described in Paper I, we constructed $0.3-1.0 \mathrm{keV}$ maps of each galaxy. We then made an initial estimate of the spatial extent of the low energy diffuse X-ray emission by eye from the $0.3-1.0$ $\mathrm{keV}$ maps assuming an elliptical distribution, estimating the centroid of the emission, the radial extent, the ellipticity, and the position angle of the emission. For some of the pre-merger systems, two distinct regions of diffuse light are seen, associated with the two galaxies in the pair, so two elliptical regions were marked and the two regions were treated separately.

We then divided these ellipses into a set of concentric elliptical annuli, and determined background-subtracted $0.3-1.0 \mathrm{keV}$ counts and photon flux surface brightness in each annuli using the ciao routine dmextract, excluding the point sources detected by the wavdetect software. For

\footnotetext{
${ }^{4}$ https://heasarc.gsfc.nasa.gov/xanadu/xspec/
}

background subtraction, we used large areas outside of the optical extent of the galaxies excluding bright point sources. All of our target galaxies have small enough angular size(s) such that we can obtain sufficient background regions on the same chip. The flux calibration was done using a $0.8 \mathrm{keV}$ monoenergetic exposure map. When multiple datasets were available, each set was calibrated individually and the results combined. We then produced radial profiles for each galaxy.

The derivation of the radial profiles was done iteratively, modifying the initial region on the sky and the annuli widths until good radial profiles were produced. We started by dividing the initial preliminary ellipse into 10 radial annuli, adding three more annuli outside of the initial radius for a total of 13 annuli. If there were too few counts to get a good radial profile with 13 annuli, we divided the initial ellipse into only five annuli, and added 2 outside the initial region for a total of 7 annuli. In some low $\mathrm{S} / \mathrm{N}$ cases, to get sufficient counts it was necessary to divide the initial ellipse into only three annuli, plus two additional annuli outside, for a total of five annuli. In total, we were able to derive radial profiles for 28 systems by this method, with 16 using 13 annuli, six using seven annuli, and and six using five annuli.

The final background-subtracted radial profiles of the diffuse emission as obtained from dmextract are displayed in Figures 5 - 7, after conversion into $0.3-1.0 \mathrm{keV}$ surface brightness in units of photons $\mathrm{s}^{-1} \mathrm{~cm}^{-2} \operatorname{arcsec}^{-2}$. In most cases, these radial profiles are centrally-peaked, but there are a few exceptions, most notably Arp 244 and Arp 299 (see Figure 6).

As a check on these results, we also obtained radial profiles using a different method. Instead of dmextract, we used the ciao routine specextract to extract the soft $(0.3-1.0 \mathrm{keV})$ X-ray spectra for each annulus. When multiple datasets are available, the "combine = yes" option was used, which calibrates each dataset individually, then the weighted spectra were coadded. The ISIS (Interactive Spectral Interpretation System) softwart5 (Houck \& Denicola 2000) was then used to derive background-subtracted $0.3-1.0 \mathrm{keV}$ counts and photon fluxes in each annuli, taking into account the calibrated response function of

\footnotetext{
${ }^{5}$ https://space.mit.edu/ASC/ISIS/
} 
the detector. These two procedures give reasonably consistent radial profiles, with the dmextract method giving lower fluxes by about a factor of 1.2 and somewhat smaller uncertainties. In the subsequent determination of the radial extent of the $\mathrm{X}$-ray emission in the galaxies and the following analysis, we used the dmextract-determined radial profiles.

Our goal in this paper is to obtain the physical size of the hot gas distribution within the galaxies, to derive electron densities and hot gas masses. An issue, however, is that how far out in the galaxy the X-ray emission can be detected depends upon the exposure time for the observations and the width of the annulus that is used. For the same annulus width, more sensitive observations can detect gas further out in the galaxy. This would give larger radii for the hot gas extent, although the gas in the outskirts may contribute little to the overall X-ray luminosity of the galaxy. This could lead to a bias in the analysis, producing larger volumes of hot gas for longer observations, which will affect the derivation of the electron densities and therefore the masses of hot gas. Because this is an archival sample, there is a large galaxy-to-galaxy variation in the observing times used.

To get around this issue, it is desirable to use a consistent definition for the radius from galaxy to galaxy. In past studies of the hot gas distribution of galaxies, a number of different methods have been used to determine the volume of hot gas. For example, Boroson. Kim. \& Fabbiano (2011) and Goulding et al. (2016) measured the extent out to where the diffuse emission equals the background. McQuinn et al. (2018) used a similar method, measuring the extent out to when the diffuse emission is detected at a $2 \sigma$ level. Other groups measured the emission within the optical D25 isophote or the optical effective radius, and used this extent as the X-ray size in deriving electron densities (Mineo et al. 2012b; Su et al. 2015; Gaspari et al.|2019). A third method was used by Strickland et al. (2004a) and Grimes et al. (2005), who used the radius which encloses a given fraction of the total $0.3-1.0 \mathrm{keV}$ flux. They find that the $90 \%$ enclosed-light fraction corresponds to an $0.3-1.0 \mathrm{keV}$ surface brightness between approximately $\sim 10^{-9}-10^{-8}$ photons $\mathrm{s}^{-1} \mathrm{~cm}^{-2} \operatorname{arcsec}^{-2}$ for their sample galaxies.

Because our dataset is so heterogeneous, af- ter some experimentation we chose to measure the radial extent out to a consistent $0.3-1.0$ $\mathrm{keV}$ surface brightness level for all of the sample galaxies. To decide on this level, we explored how the enclosed-light fraction varies with different surface brightness cutoffs, assuming that the counts within the optical B band 25 mag $\operatorname{arcsec}^{-2}$ isophote is the 'total' flux (this issue is discussed further below). Upon experimentation, we found that for most galaxies a $0.3-1.0$ $\mathrm{keV}$ surface brightness cutoff of $3 \times 10^{-9}$ photons $\mathrm{s}^{-1} \mathrm{~cm}^{-2} \operatorname{arcsec}^{-2}$ produced counts that agreed with the total counts within $10 \%$. This is consistent with the Grimes et al. (2005) and Strickland et al. (2004a) results for their 90\% enclosed-light fractions.

There were 18 systems that were detected in the MEKAL component in Paper I but had too few counts for us to derive an acceptable radial profile. For these galaxies, we derived approximate sizes by starting with the initial by-eye elliptical regions, then iteratively increasing the size of the ellipse by $30 \%$ until the galaxy is detected at the $\geq 2 \sigma$ level and the $0.3-1.0 \mathrm{keV}$ counts in the expanded ellipse equaled those in the $\mu_{\mathrm{B}}$ $=25 \mathrm{mag} \operatorname{arcsec}^{-2}$ isophote within the uncertainties. For the widely separated pre-merger pairs with two distinct regions of hot gas within the two optical galaxies, the two galaxies in the pair were treated separately in this procedure.

Four of the galaxies for which we could not find a radial profile (Arp 163, Arp 235, Arp 243, and Arp 263) were undetected in the MEKAL component in Paper I. These four galaxies are not included in any of the subsequent plots in this paper which involve quantities derived from the spatial size of the X-ray emission. Another galaxy, UGC 02238, was nominally detected in the MEKAL component at the $2.6 \sigma$ level in the spectral decomposition in Paper I, however, in the $0.3-1.0$ $\mathrm{keV}$ map it was not detected within the optical extent of the galaxy at the $2 \sigma$ level. It is also omitted from the subsequent analysis in the current paper. Another system, UGC 05189, was undetected in Paper I in the MEKAL component, however, we detected the inner disks of both galaxies in the pair at the $5 \sigma$ level in the $0.3-1.0 \mathrm{keV}$ map. The area covered by the diffuse gas is considerably smaller than the optical extent, which might explain the non-detection in the spectral decomposition. 
Except for the five systems for which we could not derive radial profiles, the Chandra $0.3-1.0$ $\mathrm{keV}$ maps of the sample galaxies are displayed in the Appendix of this paper (Figures 20 - 27). In Table 3, we provide the central coordinates, major and minor axis radii, and position angles of the final ellipses derived using the methods described above, with the dmextract-derived sizes at $3 \times 10^{-9}$ photons s ${ }^{-1} \mathrm{~cm}^{-2} \operatorname{arcsec}^{-2}$ level. Table 3 also gives the number of annuli used in the radial profile $(13,7,5$, or 1$)$. For systems with two distinct regions of diffuse emission, two ellipses are given in Table 3 . In those cases, the name of the specific galaxy in the pair associated with the particular region is identified in the second column of Table 3. When the X-ray flux only comes from one galaxy in a pair, the name of that individual galaxy is listed in Table 3 . If both galaxies in a pair are covered by a single region of diffuse emission, both names are given in the second column of Table 3. If there is only one galaxy in the system, the second column gives an alternative name for the galaxy. Table 3 also provides the point-source subtracted, background-subtracted $0.3-1.0 \mathrm{keV}$ counts in the final ellipse. Table 3 does not include UGC 02238 or the four systems without radial profiles that are undetected in the thermal component in Paper I. The final ellipses are superimposed on images of the galaxies in the Appendix of the paper.

In Figure 8, we compare the backgroundsubtracted $0.3-1.0 \mathrm{keV}$ counts obtained within the $\mu_{\mathrm{B}}=25 \mathrm{mag} \operatorname{arcsec}^{-2}$ isophote with those extracted within the Table 3 radial extents. The solid line on this plot is the one-to-one relation, and the dashed lines mark $\pm 10 \%$ differences. The systems marked by green hexagons in Figure 8 are those for which we were not able to find a radius using a set of concentric annuli. For most of the galaxies in the sample, the two measurements of the X-ray counts agree within the uncertainties with the range marked by the dotted lines. For only one system, IRAS 17208-0014, does our total counts in the $3 \times 10^{-9}$ photons $\mathrm{s}^{-1} \operatorname{arcsec}^{-2}$ isophote exceed that in the optical isophotes by $10 \%$ or more, taking into account the uncertainties (i.e., only one system lie below the bottom dotted line). For IRAS 17208-0014, the X-ray radial extent in Table 3 exceeds the optical D25 size by a factor of 1.5 , and the $0.3-1.0 \mathrm{keV}$ counts within the Table 3 ellipse are about 2.2 times those within the optical isophotes.

Taking into account the uncertainties, four systems in our sample have counts within the 'best' radii that are $60 \%-80 \%$ of the counts within the optical extent, and one (UGC 05189) has counts within the 'best' radii that are $50 \%$ of the counts in the optical isophotes. These systems lie above the top dotted line in Figure 8. Most of these systems are galaxy pairs which have two distinct regions of X-ray emission within the $\mu_{\mathrm{B}}=25 \mathrm{mag} \operatorname{arcsec}^{-2}$ isophote. Very faint diffuse emission outside of these regions may contribute to the total counts in the optical extent. This faint emission likely doesn't contribute much to the overall mass of hot gas in the system.

For all but one of our systems, in our dmextract radial profiles we can measure X-ray emission beyond the $3 \times 10^{-9}$ photons $\mathrm{s}^{-1} \mathrm{~cm}^{-2} \operatorname{arcsec}^{-2}$ isophote. For completeness, for these systems we provide the full $(2 \sigma)$ extent of the X-ray emission in another table, Table 4 . In all but 11 of these cases, the $2 \sigma$ extent of the diffuse X-ray emission is $20 \%$ or more larger than the optical D25 size. The most extreme case is Markarian 231 for which the ratio of the $2 \sigma \mathrm{X}$-ray radius divided by the maximum $\mu_{\mathrm{B}}=25 \mathrm{mag} \operatorname{arcsec}^{-2}$ radius is 2.6. For Markarian 231, the counts within the $2 \sigma$ extent are about 1.5 times those in the D25 radius. Although the measured $2 \sigma$ X-ray sizes are often larger than the D25 extent, the $0.3-1.0 \mathrm{keV}$ counts within the $2 \sigma$ radius are generally less than or consistent with the counts within the D25 radius. This means that the emission outside of D25 does not contribute significantly to the total flux.

\section{Volume and Mass of Hot Gas, Electron Densities, and Filling Factor}

We calculated the volume of hot gas for each system in the sample, assuming that the hot gas distribution has an ellipsoidal structure with the third dimension equal to the average of the other two. For these calculations, we use the X-ray sizes at the $3 \times 10^{-9}$ photons $\mathrm{s}^{-1} \operatorname{arcsec}^{-2}$ isophote as discussed above (Table 3). For the pre-merger systems for which we could measure two distinct regions of hot gas, we calculated the sum of the two volumes.

The uncertainty in the geometry of the hot 
gas likely contributes scatter to the relationships shown below. Although the true 3-dimensional distribution of the hot gas in a particular galaxy is unknown, assuming random orientations in space we can use the statistics of the observed ellipticities of the diffuse X-ray emission on the sky (Table 3 ) to make a rough estimate of the average uncertainty in the volume. The average major/minor axial ratio of the diffuse X-ray emission on the sky is 1.50 , with an rms of 0.39 . We therefore assume that the line-of-sight dimension on average will range from 1.5 times bigger than the average of the other two dimensions, to 1.5 times smaller. Thus we assume that our estimates of the volume are uncertain by a factor of 1.5 .

Using the derived volumes of hot gas, we estimated electron densities in the hot gas as a function of filling factor. For this calculation, we used the relation $\mathrm{L}_{\mathrm{X}}$ (gas) $=\Lambda \mathrm{n}_{\mathrm{e}}^{2} \mathrm{fV}$, where $\mathrm{L}_{\mathrm{X}}$ (gas) is the absorption-corrected $0.3-8.0 \mathrm{keV}$ $\mathrm{X}$-ray luminosity of the hot gas from Paper I (the MEKAL component), $\Lambda$ is the cooling function (McKee \& Cowie 1977; McCray 1987), V is the volume of gas, $n_{e}$ is the electron density, and $f$ is the filling factor. In this calculation, we assumed that the number of hydrogen atoms $\sim n_{e}$. The derived gas masses depend upon the temperature of the X-ray-emitting gas. Unfortunately, for only 15 systems were we able to obtain a fit for the gas temperature in Paper I (see Table 5 in that paper). For the remaining systems, we assumed a temperature of $0.3 \mathrm{keV}$. In Section 6.5 of this paper, we investigate how this assumption affects our results. In calculating $\mathrm{n}_{\mathrm{e}}$, we neglect X-ray emission outside of the $0.3-8.0 \mathrm{keV}$ Chandra bandpass, however, emission outside of this range may also contribute to cooling the gas. In Section 6.5, we discuss this approximation and how it depends upon temperature.

From the X-ray luminosity, the volume, and the temperature we derive $n_{e} \sqrt{f}$ for our sample galaxies; we are not able to independently determine $n_{e}$ and $f$. We find that $n_{e} \sqrt{f}$ ranges from $1.1 \times 10^{-3}-2.2 \times 10^{-2} \mathrm{~cm}^{-3}$, similar to the values found by Mineo et al. (2012b) for their spirals. Accounting for the uncertainty in volume and conservatively assuming a factor of two uncertainty in $\mathrm{L}_{\mathrm{X}}$ (gas) (due in part to uncertainties in separating the thermal and non-thermal emission; see Section 5.3 in Paper I), propagation of errors implies that our estimates of $n_{e} \sqrt{f}$ are uncertain by a factor of 1.8 on average.

The radiative cooling times for the hot gas (i.e., total thermal energy divided by $\mathrm{L}_{\mathrm{X}}$ (gas)) in these galaxies range from 16 to $700 \mathrm{Myrs}$, with a median time of about 60 Myrs. These are similar to the Mineo et al. (2012b) estimates for disk galaxies. We then calculated the mass of the hot $\mathrm{X}$ ray-emitting gas $M_{X}$ (gas) $=m_{p} n_{e} V$, when $m_{p}$ is the mass of a proton. Accounting for the uncertainties in $\mathrm{V}$ and $\mathrm{n}_{\mathrm{e}}$, we adopt an uncertainty in our estimates of $\mathrm{M}_{\mathrm{X}}$ (gas) of a factor of two, not including the uncertainty in the filling factor.

\section{Correlation Analysis}

From the Chandra data we derived a set of parameters for our sample galaxies, including the volume of hot gas, $\mathrm{n}_{\mathrm{e}} \sqrt{f}, \mathrm{~L}_{\mathrm{X}}$ (gas), and $\mathrm{M}_{\mathrm{X}}$ (gas). From data at other wavelengths, we have another set of values for our galaxies, including SFR, SFE, $\mathrm{L}_{\mathrm{FIR}}, \mathrm{L}_{\mathrm{K}}, \mathrm{L}_{\mathrm{FIR}} / \mathrm{L}_{\mathrm{K}},[3.6]-[24]$, $\mathrm{F}_{60} / \mathrm{F}_{100}, \mathrm{M}_{\mathrm{HI}} / \mathrm{M}_{\mathrm{H}_{2}}$, and the merger stage. Combining these two sets, we derive additional parameters, including $\mathrm{L}_{\mathrm{X}}$ (gas)/SFR, $\mathrm{M}_{\mathrm{X}}$ (gas)/SFR, and $\mathrm{M}_{\mathrm{X}}$ (gas) $/\left(\mathrm{M}_{\mathrm{H}_{2}}+\mathrm{M}_{\mathrm{HI}}\right)$. In this section, we correlate these parameters against each other, and calculate the best-fit linear $\log$ vs. $\log$ relations for various combinations of these parameters. In Paper I, we found that some trends change at low SFRs, so we did these fits for two cases: the full range of SFRs and the subset of systems with SFR $>1 \mathrm{M}_{\odot} \mathrm{yr}^{-1}$.

For each relation, we calculated the root mean square (rms) deviation from the best-fit line and the Spearman rank order coefficient. These values are compiled in Table 5, along with the best-fit parameters. For comparison with the Spearman coefficients, Table 5 also provides Pearson correlation coefficients, which assumes a linear relationship between the two parameters. The two types of correlation coefficients agree fairly well for our sample (see Table 5). In Table 5, we classified the relations, into "strong correlation", "weak correlation", or "no correlation". We defined a "strong correlation" as one in which the Spearman coefficient is greater than 0.55 (i.e., $\leq 0.1 \%$ likelihood of happening by chance), and a "strong anti-correlation" is one in which the Spearman coefficient is less than -0.55 . A "weak correlation" 
is one in which the Spearman coefficient is between 0.35 and 0.55 , where 0.35 corresponds to a $\sim 5 \%$ probability of happening by chance. A "weak anti-correlation" implies a Spearman coefficient between -0.35 and -0.55 , and "no correlation" means a Spearman coefficient between -0.35 and 0.35 .

The most important of the correlations are plotted in Figures $9-19$. For convenience, when a plot is shown, the number of the figure which displays each correlation is provided in Table 5 along with the best-fit parameters and the Spearman/Pearson coefficients. For clarity of presentation, we do not include errorbars on the plots. As discussed above, we estimate that our values of $\mathrm{M}_{\mathrm{X}}$ (gas) are uncertain by about a factor of two. This means that the uncertainty in $\log \left(\mathrm{M}_{\mathrm{X}}(\right.$ gas $\left.)\right)$ is about 0.3 dex. The rms uncertainties on some of the fits involving $\log \left(\mathrm{M}_{\mathrm{X}}\right.$ (gas)) are close to or slightly larger than this estimate (see Table 5), so the uncertainty in $\mathrm{M}_{\mathrm{X}}$ (gas) may be a limiting factor in this analysis. Because the uncertainty in the $\mathrm{CO} / \mathrm{H}_{2}$ ratio is potentially an even larger factor, we do the correlations for both $\mathrm{CO} / \mathrm{H}_{2}$ ratios. This provides a test of whether the results are biased by the choice of $\mathrm{CO} / \mathrm{H}_{2}$ ratios.

As another test, we also ran the correlation analysis using radii and volumes determined from the specextract/ISIS radial profiles rather than dmextract. The best-fit relations and correlation coefficients changed slightly but the basic conclusions of the paper were unchanged. The relations given below were derived from the dmextract results.

To see if our results are affected by the inclusion of Seyfert galaxies in the sample, we also calculated the correlations excluding the AGNs. The correlation coefficients tend to be somewhat smaller with the smaller sample, however, the basic results do not change and the important correlations discussed below still hold.

\subsection{Relations with Volume and with $\mathbf{n}_{\mathrm{e}}$}

In the top row of Figure 9, we plot the volume of hot gas as a function of merger stage, $\mathrm{L}_{\mathrm{X}}$ (gas)/SFR, and SFR. The bottom row of panels in this figure displays the ratio of the maximum radial extent of the X-ray emission to the maximum optical size as measured by the $\mathrm{B}$ band
$25 \mathrm{mag} \operatorname{arcsec}^{-2}$ isophote against merger stage, $\mathrm{L}_{\mathrm{X}}$ (gas)/SFR, and SFR. The first column of Figure 9 shows that stage 3 and stage 4 mergers tend to have large X-ray sizes and large X-ray/optical size ratios. This is a consequence of the bias towards higher SFRs in the mid-merger stages. The second column shows little correlation between the volume of hot gas and $\mathrm{L}_{\mathrm{X}}$ (gas)/SFR, or between the X-ray to optical size and $\mathrm{L}_{\mathrm{X}}$ (gas)/SFR. Systems in which the X-ray extent exceeds that in the optical tend to have high SFRs (Figure 9; bottom right panel), but with a lot of scatter.

In Figure 9, the strongest correlation is seen in the top right panel: larger volumes of hot X-rayemitting gas are found in higher SFR systems. For the full set of galaxies, the best-fit slope is less than one. However, once low SFR systems are excluded the slope is consistent with one. Thus low SFR systems tend to have larger volumes than expected based on their SFRs.

We have marked the location of the late-stage merger NGC 1700 on the two upper right panels in Figure 9. It stands out in the sample for having a large volume of hot gas, compared to its SFR. This galaxy has a high $\mathrm{L}_{\mathrm{K}}$ and a low SFR, with an elliptical-like appearance and tidal debris. It may be a system for which virialized hot gas in the gravitational potential contributes significantly to the observed diffuse X-ray-emitting gas. This system is discussed further in Section 7.4.

In Figure 10, we plot the volume of hot gas (top row) and the X-ray/optical size ratio (bottom row) against the SFE (first and second columns) and the $\mathrm{F}_{60} / \mathrm{F}_{100}$ ratio (last column). The first column utilizes a constant $\mathrm{CO} / \mathrm{H}_{2}$ ratio, while the second uses the variable $\mathrm{CO} / \mathrm{H}_{2}$ ratio. This Figure shows that the systems with large volumes tend to have large SFEs (when a variable $\mathrm{CO} / \mathrm{H}_{2}$ ratio is used). However, this is a very weak correlation with a lot of scatter; some systems with large SFEs have only moderate volumes and size ratios.

No significant correlation is seen between volume and $\mathrm{F}_{60} / \mathrm{F}_{100}$ (Figure 10, upper right), in spite of the fact that volume is correlated with SFR (Figure 9), and $\mathrm{F}_{60} / \mathrm{F}_{100}$ is correlated with SFR (Figure 2). However, the correlation between $\mathrm{F}_{60} / \mathrm{F}_{100}$ and SFR is weak, with considerable scatter. The sample galaxies only cover a small range in $\log \mathrm{F}_{60} / \mathrm{F}_{100}(0.3 \mathrm{dex})$ while the $\mathrm{SFR}$ varies over two orders of magnitude. This means that 
uncertainties in the IRAS fluxes can make it difficult to detect a correlation. Instead of being directly dependent on SFR itself, theoretical models (e.g., Desert, Boulanger, \& Puget 1990) suggest that the $\mathrm{F}_{60} / \mathrm{F}_{100}$ ratio depends on the intensity of the ISRF. The average ISRF in a galaxy can vary greatly for a given global SFR of the galaxy, depending upon the spatial density of young stars. In contrast, the volume of hot gas in these galaxies depends directly upon the total number of young stars rather than on the spatial density of those stars.

The upper left panel of Figure 11 shows that the volume of hot gas is well-correlated with $\mathrm{L}_{\mathrm{X}}$ (gas). The slope in this log-log plot is close to one. In the right panel of Figure 11, the volume is plotted against the derived $n_{e} \sqrt{f}$. There is a weak trend of decreasing $n_{\mathrm{e}} \sqrt{f}$ with increasing volume.

In the lower left panel of Figure 11, we show that the volume is also correlated with $\mathrm{L}_{\mathrm{K}}$. However, the correlation is weaker than for volume vs. SFR, and the scatter is larger (Table 5). This supports the idea that in this sample of galaxies the volume of hot gas is largely determined by the number of young stars, with the correlation of volume with $\mathrm{L}_{\mathrm{K}}$ being a by-product of the $\mathrm{SFR}-\mathrm{L}_{\mathrm{K}}$ correlation.

The volume of hot gas is weakly correlated with $\mathrm{L}_{\mathrm{FIR}} / \mathrm{L}_{\mathrm{K}}$ (bottom right panel of Figure 11) and with [3.6] - [24] (Table 5). As noted earlier, both [3.6] - [24] and $\mathrm{L}_{\mathrm{FIR}} / \mathrm{L}_{\mathrm{K}}$ are approximate measures of the sSFR. This correlation may be a consequence of the correlation between volume and SFR, since sSFR tends to increase with increasing SFR for this sample (see Figure 2 and Table 5). Notice that NGC 1700 is particularly discrepant in these plots compared to the other galaxies, with a low sSFR (i.e., low [3.6] - [24] and low $\mathrm{L}_{\mathrm{FIR}} / \mathrm{L}_{\mathrm{K}}$ ) and a large volume of hot gas.

In Figure 12, SFR is plotted against $\mathrm{n}_{\mathrm{e}} \sqrt{f}$ (upper left panel), $\mathrm{F}_{60} / \mathrm{F}_{100}$ against $\mathrm{n}_{\mathrm{e}} \sqrt{f}$ (upper right panel), SFE with constant $\mathrm{CO} / \mathrm{H}_{2}$ ratio vs. $\mathrm{n}_{\mathrm{e}} \sqrt{f}$ (middle left), SFE with a variable $\mathrm{CO} / \mathrm{H}_{2}$ ratio vs. $\mathrm{n}_{\mathrm{e}} \sqrt{f}$ (middle right), and [3.6] - [24] vs. $\mathrm{n}_{\mathrm{e}} \sqrt{f}$. No trends are seen in these five panels.

In the lower right panel of Figure 12, we plot the derived ratio $\mathrm{M}_{\mathrm{X}}$ (gas) $/ \mathrm{L}_{\mathrm{X}}$ (gas) for the 15 galaxies with temperature measurements. The blue solid line on this plot is the relation assuming a constant temperature of $0.3 \mathrm{keV}$. From the equations given in Section $5, \mathrm{M}_{\mathrm{X}}$ (gas) $/ \mathrm{L}_{\mathrm{X}}$ (gas) $=\mathrm{m}_{\mathrm{P}} /\left(\Lambda \mathrm{n}_{\mathrm{e}} \mathrm{f}\right)$, so the conversion from $\mathrm{L}_{\mathrm{X}}$ (gas) to $\mathrm{M}_{\mathrm{X}}$ (gas) is a function of $n_{e}$, with $M_{X}($ gas $) / L_{X}($ gas $) \propto 1 / n_{e}$ if the temperature and filling factor are constant. The 15 data points lie above the blue line in this plot because they have temperatures higher than 0.3 $\mathrm{keV}$ (see Section 6.5), and $\Lambda$ decreases as temperature increases. The question of the assumed electron temperature is discussed further in Section 6.5 .

\section{2. $\mathrm{M}_{\mathrm{X}}($ gas $) / \mathrm{SFR}$ vs. Other Properties}

The mass of hot gas is strongly correlated with SFR (Figure 13, upper left). For SFR > $1 \mathrm{M}_{\odot} \mathrm{yr}^{-1}$, the slope of $\log \mathrm{M}_{\mathrm{X}}$ (gas) vs. $\log \mathrm{SFR}$ is consistent with one. However, the relationship flattens when lower SFR systems are included, suggesting an excess of hot gas in low SFR systems. Even when NGC 1700 is excluded, this flattening is seen. We note that the other two galaxies with low SFR and high $\mathrm{M}_{\mathrm{X}}$ (gas)/SFR in this figure, NGC 2865 and NGC 5018, both have moderately high $\mathrm{K}$ band luminosities (Table 1). As with NGC 1700, virialized gas in the gravitational potential may be contributing to the observed hot gas in these galaxies (see Paper I for detailed discussions of these galaxies). Unfortunately, our sample only has a few low SFR, high $\mathrm{L}_{\mathrm{K}}$ systems, so separating out this additional component to the hot gas is is uncertain.

$\mathrm{M}_{\mathrm{X}}$ (gas) is also correlated with $\mathrm{L}_{\mathrm{K}}$ (Figure 13, upper right). However, this relation has a lower correlation coefficient than $\mathrm{M}_{\mathrm{X}}$ (gas) vs. SFR. This suggests that the $\mathrm{M}_{\mathrm{X}}$ (gas)- $\mathrm{L}_{\mathrm{K}}$ relation is a consequence of the SFR- $\mathrm{L}_{\mathrm{K}}$ correlation for our sample galaxies, and the hot gas in most of our galaxies is mainly due to SFR rather than older stars.

When both $\mathrm{M}_{\mathrm{X}}$ (gas) and SFR are normalized by a tracer of stellar mass, $\mathrm{L}_{\mathrm{K}}$, they still show a strong correlation (Table 5). This indicates that the relation between $\mathrm{SFR}$ and $\mathrm{M}_{\mathrm{X}}$ (gas) is not simply a richness effect. In contrast, when both $\mathrm{M}_{\mathrm{X}}$ (gas) and $\mathrm{L}_{\mathrm{K}}$ are normalized by SFR, the correlation is significantly weaker (Table 5 ). This again implies that $\mathrm{M}_{\mathrm{X}}$ (gas) is more closely tied to young stars than to old stars.

The correlation between $\mathrm{M}_{\mathrm{X}}$ (gas) and $\mathrm{SFR}$ is displayed in another way in the bottom left panel 
of Figure 13, where we show $\mathrm{M}_{\mathrm{X}}$ (gas)/SFR vs. SFR. Although a weak anti-correlation is seen for the full sample, once systems with $\mathrm{SFR}<1$ $\mathrm{M}_{\odot} \mathrm{yr}^{-1}$ are removed no correlation is seen and the rms scatter is relatively small ( $0.37 \mathrm{dex})$. This is close to the expected scatter based on the uncertainty in $\mathrm{M}_{\mathrm{X}}$ (gas) alone, which supports the contention that processes associated with a young stellar population are the main factors responsible for the hot gas in these galaxies, at least when low SFR systems are excluded.

$\mathrm{M}_{\mathrm{X}}$ (gas)/SFR is plotted against $\mathrm{L}_{\mathrm{K}}$ in the lower right panel of Figure 13. A very weak trend is seen when low SFR systems are excluded. The post-merger NGC 1700 stands out as having a high $\mathrm{M}_{\mathrm{X}}$ (gas)/SFR. After NGC 1700, the next two highest $\mathrm{M}_{\mathrm{X}}$ (gas)/SFR galaxies in this plot, NGC 2865 and NGC 5018, are both stage 7 merger remnants with moderately high $\mathrm{L}_{\mathrm{K}}$ and low sSFR. In contrast to these three galaxies, galaxies with low $\mathrm{K}$ band luminosities $\left(\leq 10^{10} \mathrm{~L}_{\odot}\right)$ have moderately low $\mathrm{M}_{\mathrm{X}}$ (gas)/SFR values, though not extreme. In Paper I, we found that low $\mathrm{L}_{\mathrm{K}}$ systems have low $L_{X}$ (gas)/SFR. Now, we are able to show that $\mathrm{M}_{\mathrm{X}}$ (gas)/SFR is also somewhat low for these systems. This may indicate escape of hot gas from lower gravitational fields. However, only a few galaxies fall in this range, so the statistics are very uncertain.

In the upper left and upper middle panels of Figure 14, weak anti-correlations are seen between $\mathrm{M}_{\mathrm{X}}$ (gas)/SFR and SFE, but these trends disappear for the variable $\mathrm{CO} / \mathrm{H}_{2}$ ratio when low SFR systems are not included. The fact that our dataset is incomplete in CO makes these conclusions somewhat uncertain.

$\mathrm{M}_{\mathrm{X}}$ (gas)/SFR is anti-correlated with the two tracers of sSFR, [3.6] - [24] and $\mathrm{L}_{\mathrm{FIR}} / \mathrm{L}_{\mathrm{K}}$ (Figure 14 , upper right and lower left panel, respectively). However, when low SFR systems are excluded the trend with [3.6] - [24] weakens and the trend with $\mathrm{L}_{\mathrm{FIR}} / \mathrm{L}_{\mathrm{K}}$ disappears. This again suggests that low sSFR systems sometimes have excess hot gas.

$\mathrm{M}_{\mathrm{X}}$ (gas)/SFR is shown to be strongly anticorrelated with $\mathrm{F}_{60} / \mathrm{F}_{100}$ for the full sample (Figure 14, lower middle panel). This trend is weakened when only the high SFR sample is included, but is still detected. The cause of this anticorrelation is uncertain; some possible interpretations are discussed in Section 7.2. In addi- tion, a weak anti-correlation is visible between $\mathrm{M}_{\mathrm{X}}$ (gas)/SFR and $\mathrm{n}_{\mathrm{e}} \sqrt{f}$, particularly when low SFR systems are omitted (lower right panel Figure 14). In contrast, $\mathrm{M}_{\mathrm{X}}$ (gas)/SFR is not correlated with either the volume or the $\mathrm{H} \mathrm{I}$-to- $\mathrm{H}_{2}$ ratio (Table 5).

\subsection{Ratio of Mass Hot Gas to Mass Cold Gas vs. Other Properties}

In Figure 15, the ratio of the mass of hot X-rayemitting gas to the mass of cold gas $\left(\mathrm{H} \mathrm{I}+\mathrm{H}_{2}\right)$ is plotted against SFR (top row) and SFE (bottom row). The left panel in each row was calculated using a constant $\mathrm{CO} / \mathrm{H}_{2}$ ratio, while the right panel was calculated with a variable $\mathrm{CO} / \mathrm{H}_{2}$. Figure 15 shows that $\mathrm{M}_{\mathrm{X}}$ (gas) $/\left(\mathrm{M}_{\mathrm{H}_{2}}+\mathrm{M}_{\mathrm{HI}}\right)$ increases with increasing SFR, with a better correlation when a variable $\mathrm{CO} / \mathrm{H}_{2}$ ratio is used. A higher Spearman coefficient and a steeper relation are found when the low SFR systems are omitted. The slope is consistent with one when a variable $\mathrm{CO} / \mathrm{H}_{2}$ ratio is used and low SFR systems are omitted. The flatter relation when low SFR systems are included again points to excess $M_{X}$ (gas) for low SFR systems.

A large amount of scatter is evident in a plot of $\mathrm{M}_{\mathrm{X}}$ (gas) $/\left(\mathrm{M}_{\mathrm{H}_{2}}+\mathrm{M}_{\mathrm{HI}}\right)$ vs. $\mathrm{SFE}$ (Figure 15, bottom row), but a reliable correlation is present when a variable $\mathrm{CO} / \mathrm{H}_{2}$ ratio is used. The lack of a full set of $\mathrm{CO}$ data makes these results uncertain.

The scatter in $\mathrm{M}_{\mathrm{X}}$ (gas) $/\left(\mathrm{M}_{\mathrm{H}_{2}}+\mathrm{M}_{\mathrm{HI}}\right)$ may be due in part to variations in the stellar mass. In Figure $16, \mathrm{M}_{\mathrm{X}}$ (gas) $/\left(\mathrm{M}_{\mathrm{H}_{2}}+\mathrm{M}_{\mathrm{HI}}\right)$ is plotted against $\mathrm{L}_{\mathrm{K}}$ (top panel) and $\mathrm{F}_{60} / \mathrm{F}_{100}$ (bottom panel). In the left column, the quantities were calculated using a constant $\mathrm{CO} / \mathrm{H}_{2}$ ratio, while the right panel was calculated with a variable $\mathrm{CO} / \mathrm{H}_{2}$. A weak correlation is visible in the upper right panel when low SFR galaxies are excluded and a variable $\mathrm{CO} / \mathrm{H}_{2}$ ratio is used. The two lowest $\mathrm{L}_{\mathrm{K}}$ systems have moderately low $\mathrm{M}_{\mathrm{X}}$ (gas) $/\left(\mathrm{M}_{\mathrm{H}_{2}}\right.$ $\left.+\mathrm{M}_{\mathrm{HI}}\right)$, and the highest $\mathrm{M}_{\mathrm{X}}$ (gas) $/\left(\mathrm{M}_{\mathrm{H}_{2}}+\mathrm{M}_{\mathrm{HI}}\right)$ system, NGC 6240, has a very high $\mathrm{L}_{\mathrm{K}}$. In the lower panels of Figure 16, weak correlations between $\mathrm{M}_{\mathrm{X}}$ (gas) $/\left(\mathrm{M}_{\mathrm{H}_{2}}+\mathrm{M}_{\mathrm{HI}}\right)$, and $\mathrm{F}_{60} / \mathrm{F}_{100}$ are seen, but only if low SFR systems are excluded.

Correlations are visible between $\mathrm{M}_{\mathrm{X}}$ (gas) $/\left(\mathrm{M}_{\mathrm{H}_{2}}\right.$ $+\mathrm{M}_{\mathrm{HI}}$ ) and our two tracers of sSFR (Figure 17), especially when low SFR systems are excluded and 
a variable $\mathrm{CO} / \mathrm{H}_{2}$ ratio is used. The steepening of the slope when low SFR systems are excluded again signals possible excess of hot gas in low SFR systems.

\subsection{Merger Stage vs. Gas Properties}

We plot the inferred mass of X-ray-emitting gas $\mathrm{M}_{\mathrm{X}}$ (gas) against merger stage in the top left panel of Figure 18. The mid-merger stages have higher quantities of hot gas, on average, than the early or late stages. However, this is largely due to the fact that the mid-merger galaxies tend to have higher SFRs. When the mass of hot gas is normalized by the SFR (Figure 18, top right), no strong trend is seen. The stage 7 galaxy NGC 1700 stands out as having a high $\mathrm{M}_{\mathrm{X}}$ (gas)/SFR. The next two highest $\mathrm{M}_{\mathrm{X}}$ (gas)/SFR systems, the stage 7 galaxies NGC 2865 and NGC 5018, also have low sSFR.

The bottom row of Figure 18 compares the merger stage with the ratio of hot gas to cold gas $\mathrm{M}_{\mathrm{X}}$ (gas) $/\left(\mathrm{M}_{\mathrm{H}_{2}}+\mathrm{M}_{\mathrm{HI}}\right)$, using the standard Galactic $\mathrm{CO} / \mathrm{H}_{2}$ ratio (left panel) or the variable $\mathrm{CO} / \mathrm{H}_{2}$ ratio (right panel). Stages 3 and 4 tend to have proportionally more hot gas. This is likely a consequence of the fact that galaxies in those stages tend to have higher SFRs. Because of the inhomogeneity of the sample, the small number of systems in each merger stage, and the lack of a full set of $\mathrm{CO}$ data, trends with merger stage in our sample are uncertain. In these plots, the galaxy with the highest $\mathrm{M}_{\mathrm{X}}$ (gas) $/\left(\mathrm{M}_{\mathrm{H}_{2}}+\mathrm{M}_{\mathrm{HI}}\right)$ is $\mathrm{NGC}$ 6240. NGC 1700 is not plotted in the bottom row of Figure 18 because it lacks a full set of CO data.

\subsection{Gas Temperature}

As mentioned earlier, our derivations of electron density and $\mathrm{M}_{\mathrm{X}}$ (gas) depend upon the assumed temperature, and temperatures are available for only 15 of our sample galaxies from the X-ray spectra (see Paper I). For the remaining galaxies, we assumed a constant temperature of $0.3 \mathrm{keV}$. For comparison, for the 15 systems for which temperatures are available in Paper I, kT ranges from $0.37 \mathrm{keV}$ to $1.0 \mathrm{keV}$. In some cases, we were able to use a two-temperature model for the hot gas; in those cases, we used the luminosityweighted temperature in the subsequent analysis. For comparison, Mineo et al. (2012b) found lower temperatures on average for their sample galax- ies (mean of $0.24 \mathrm{keV}$ for single-temperature models). The derived temperatures depend upon the assumptions used in modeling the X-ray spectrum, including how the power law component is modeled, so they are somewhat uncertain (see Paper I). Because our sample is an archive-selected sample, there is a selection bias in the subset of galaxies with derived temperatures. Compared to the galaxies in the sample without measured temperatures, the galaxies with temperatures tend to have longer, more sensitive exposures, and they tend to be more extreme systems with higher luminosities. In contrast, the Mineo et al. (2012b) sample, with lower temperatures on average, contains more normal spiral galaxies and irregulars as well as some mergers. We therefore assume the more modest temperature of $0.3 \mathrm{keV}$ for our galaxies without temperature measurements, assuming that they are less extreme than the other systems. However, this is quite uncertain.

To test whether our conclusions are affected by our assumption of $0.3 \mathrm{keV}$ for the galaxies without derived temperatures, we re-ran our correlation analysis with four alternative assumptions. First, we re-ran the analysis using a constant $\mathrm{kT}=0.3$ $\mathrm{keV}$ for all the galaxies, even those for which we have a direct measure of the temperature. Second, we did the calculations assuming a constant $\mathrm{kT}=$ $0.6 \mathrm{keV}$ for all galaxies.

Third, we re-ran the analysis assuming that the temperature is correlated with SFR. In Paper I we did not find any correlation of temperature with SFR. Mineo et al. (2012b) also did not find a correlation between temperature and SFR for their sample of star-forming galaxies. However, Grimes et al. (2005) noted that the ULIRGs in their sample tend to have higher temperatures, up to about $0.8 \mathrm{keV}$. Therefore, as a limiting case to investigate how temperature may potentially affect our results, we assume that $\log \mathrm{T}_{\mathrm{X}}$ increases linearly with $\log \mathrm{SFR}$, and we set $\mathrm{kT}=0.2 \mathrm{keV}$ for the systems with the lowest SFRs $\left(0.1 \mathrm{M}_{\odot} \mathrm{yr}^{-1}\right)$, increasing to $1.0 \mathrm{keV}$ for systems with $\mathrm{SFR}=100$ $\mathrm{M}_{\odot} \mathrm{yr}^{-1}$.

As a fourth test, we investigated how our results changed if we assumed that the temperature depends upon $\mathrm{L}_{\mathrm{X}}$ (gas) rather than on SFR. In contrast to actively star-forming galaxies, ellipticals show a steep relation between $\mathrm{L}_{\mathrm{X}}($ gas $)$ and temperature of $\mathrm{L}_{\mathrm{X}}($ gas $) \propto \mathrm{T}_{\mathrm{X}}{ }^{4.5}$ 
(Goulding et al. 2016). As a limiting case, we assumed that $\mathrm{L}_{\mathrm{X}}(\mathrm{gas}) \propto \mathrm{T}_{\mathrm{X}}{ }^{4.5}$ as found for ellipticals (Goulding et al. 2016). Assuming a temperature of $0.2 \mathrm{keV}$ for the galaxies with the lowest $\mathrm{L}_{\mathrm{X}}$ (gas) in our sample, this gives $1.0 \mathrm{keV}$ for the highest $\mathrm{L}_{\mathrm{X}}$ (gas) system. This is a more extreme range than typically found for star-forming galaxies, thus it is a limiting case.

For each of the above cases, we also explored how our results change when we include a correction from the observed $0.3-8.0 \mathrm{keV} \mathrm{L}_{\mathrm{X}}$ (gas) to the bolometric luminosity of the gas including light outside of the $0.3-8.0 \mathrm{keV}$ Chandra window. This conversion is a function of temperature. Using the PIMMS6 software, we find that $\mathrm{L}_{\text {bol }}($ gas $) / \mathrm{L}_{\mathrm{X}}$ (gas) $(0.3-8.0 \mathrm{keV})$ drops from 2.39 at $0.3 \mathrm{keV}$ to 1.39 at $1.0 \mathrm{keV}$.

In re-running the correlation analysis, we find that the basic conclusions of this paper do not change dramatically with these different assumptions about the temperature. The Spearman coefficients and the best-fit relations change slightly with different assumptions about the temperature, but the basic conclusions remain the same. For a few of the relations that have correlation coefficients near our 'weak'/'none' boundary or our 'strong'/'weak' boundary, small changes in the correlation coefficient may reclassify the relation. The most notable case is the very weak correlation between $\mathrm{M}_{\mathrm{X}}$ (gas)/SFR and $\mathrm{L}_{\mathrm{K}}$, which drops below the cutoff for a 'weak' correlation for some of these alternative cases, but increases slightly in significance for the linear $\log \left(\mathrm{T}_{\mathrm{X}}\right)-\log (\mathrm{SFR})$ case including the correction for light outside of the Chandra bandpass. This emphasizes that the $\mathrm{M}_{\mathrm{X}}$ (gas)/SFR $-\mathrm{L}_{\mathrm{K}}$ correlation is very marginal, and more data is needed to confirm or refute it.

For most of the relations discussed above, however, although the correlation coefficients change slightly with different assumptions about the temperatures, the classification of the relation does not change. Thus the conclusions of this paper are not strongly influenced by our lack of temperature measurements.

${ }^{6}$ Portable Interactive Multi-Mission Simulator; http://asc.harvard.edu/toolkit/pimms.jsp

\section{Discussion}

We calculated the volume, mass, and electron density of the hot X-ray-emitting gas in our sample galaxies, and compared with other properties of the galaxies, including the SFR, $\mathrm{L}_{\mathrm{K}}$, the mass of cold gas, and the SFE. We have searched for correlations between a large number of variables, and discovered several new correlations and anticorrelations in our data. These, and many apparent non-correlations, are listed in Table 5 .

\subsection{Volume and $M_{X}$ (gas) vs. SFR and $L_{K}$}

Some of the most important correlations are:

(1) The volume of hot gas increases as the SFR goes up, with a high correlation coefficient (Figure 9). When galaxies with SFR $<1 \mathrm{M}_{\odot} \mathrm{yr}^{-1}$ are excluded, the slope of the best fit log volume - log SFR line is $0.97 \pm$ 0.15 (Figure 9). Including low SFR systems flattens this relation.

(2) The volume of hot gas is also correlated with $\mathrm{L}_{\mathrm{K}}$, but with a smaller correlation coefficient (Figure 11).

(3) The volume of hot gas also correlates with SFE, $\mathrm{L}_{\mathrm{FIR}} / \mathrm{L}_{\mathrm{K}}$, and [3.6] - [24], but only weakly (Figures 10 and 11, and Table 5).

(4) There is a strong correlation between $\mathrm{M}_{\mathrm{X}}$ (gas) and SFR (Figure 13). The slope of the log-log plot is $0.88 \pm 0.10$ when low SFR galaxies are excluded, consistent with a simple $\mathrm{M}_{\mathrm{X}}($ gas $) \propto \mathrm{SFR}$ relation. This relation flattens when low SFR systems are included.

(5) $\mathrm{M}_{\mathrm{X}}$ (gas) is also correlated with $\mathrm{L}_{\mathrm{K}}$ (Figure 13), but with a lower correlation coefficient than $\mathrm{M}_{\mathrm{X}}$ (gas) and SFR.

(6) As the SFR increases, $\mathrm{M}_{\mathrm{X}}$ (gas) $/\left(\mathrm{M}_{\mathrm{H}_{2}}+\right.$ $\mathrm{M}_{\mathrm{HI}}$ ) goes up (Figure 15), especially when a variable $\mathrm{CO} / \mathrm{H}_{2}$ ratio is used and when low SFR systems are excluded. For the latter case, the correlation is strong and the slope of the log-log plot is consistent with one.

(7) $\mathrm{M}_{\mathrm{X}}($ gas $) /\left(\mathrm{M}_{H_{2}}+\mathrm{M}_{H I}\right)$ is weakly correlated with $\mathrm{L}_{\mathrm{K}}$ when a variable $\mathrm{CO} / \mathrm{H}_{2}$ ratio is used (Figure 16). 
(8) There is a trend of increasing $\mathrm{M}_{\mathrm{X}}$ (gas) $/\left(\mathrm{M}_{\mathrm{H}_{2}}\right.$ $+\mathrm{M}_{\mathrm{HI}}$ ) ratio with increasing $\mathrm{SFE}$, especially when a variable $\mathrm{CO} / \mathrm{H}_{2}$ ratio is used (Figure 15). This trend is weaker than the relation with SFR.

(9) $\mathrm{M}_{\mathrm{X}}($ gas $) /\left(\mathrm{M}_{\mathrm{H}_{2}}+\mathrm{M}_{\mathrm{HI}}\right)$ is weakly correlated with $\mathrm{F}_{60} / \mathrm{F}_{100}$ (Figure 16).

For high SFR systems, the linear relations between volume and SFR, and between $\mathrm{M}_{\mathrm{X}}$ (gas) and SFR, can be explained in a straight-forward manner: a larger SFR means more supernovae and more stellar winds, which produce a larger volume of hot gas and a larger $\mathrm{M}_{\mathrm{X}}$ (gas). For galaxies with $\mathrm{SFR}>1 \mathrm{M}_{\odot} \mathrm{yr}^{-1}$, hot gas associated with star formation dominates $\mathrm{M}_{\mathrm{X}}$ (gas), and any contribution from processes associated with the older stellar population is negligible. However, for galaxies with lower SFRs and high K band luminosities (and therefore low sSFRs) we find evidence for excess hot gas relative to the linear $\mathrm{M}_{\mathrm{X}}$ (gas)-SFR relation. This may be due to contributions to the $\mathrm{X}$-ray-emitting hot gas from other sources, perhaps mass loss from older stars that has been virialized in the gravitational potential.

The weaker correlation between volume and SFE compared to volume vs. SFR is accounted for by the fact that some high SFE systems have only moderate SFRs, and it is the SFR that controls the number of supernovae and the amount of stellar wind, not the SFE. The weakness of the correlation between volume and the SSFR as measured by [3.6] - [24] and $\mathrm{L}_{\mathrm{FIR}} / \mathrm{L}_{\mathrm{K}}$ may be explained in a similar manner.

The correlations between $\mathrm{L}_{\mathrm{K}}$ and the hot gas mass, and between $\mathrm{L}_{\mathrm{K}}$ and the volume of hot gas, may be indirect results of the correlation between SFR and $\mathrm{L}_{K}$. The SFR- $\mathrm{L}_{K}$ correlation, in turn, is a consequence of the fact that most of the galaxies in our sample are star-forming galaxies on the galaxy main sequence. Because the volume- $\mathrm{L}_{\mathrm{K}}$ and $\mathrm{M}_{\mathrm{X}}(\mathrm{gas})-\mathrm{L}_{\mathrm{K}}$ correlations are weaker than the volume-SFR and $\mathrm{M}_{\mathrm{X}}$ (gas)-SFR correlations, we conclude that star formation is more directly responsible for the hot gas, not the older stellar population.

The strong correlation between the hot-to-cold gas mass ratio and the SFR, in contrast to the weak correlation between the hot-to-cold gas mass ratio and $\mathrm{L}_{\mathrm{K}}$, confirms that the younger stellar population is primarily responsible for the hot gas, not older stars. The amount of hot gas in our galaxies is small compared to the amount of colder gas (see Table 2), so conversion of colder material into hot gas affects the numerator in $\mathrm{M}_{\mathrm{X}}$ (gas) $/\left(\mathrm{M}_{\mathrm{H}_{2}}+\mathrm{M}_{\mathrm{HI}}\right)$ but not noticeably the denominator. The higher the SFR, the more hot gas that is produced, thus the $\mathrm{M}_{\mathrm{X}}$ (gas) $/\left(\mathrm{M}_{\mathrm{H}_{2}}+\right.$ $\mathrm{M}_{\mathrm{HI}}$ ) ratio is directly correlated with the SFR. The linear $\log \mathrm{M}_{\mathrm{X}}$ (gas)/( $\left.\mathrm{M}_{\mathrm{H}_{2}}+\mathrm{M}_{\mathrm{HI}}\right)$ vs. $\log \mathrm{SFR}$ relation for high SFR systems provides additional support for the idea that the hot gas is mainly due to young stars in these galaxies. The flattening of this relation at lower SFR again indicates excess hot gas in low SFR, low sSFR systems.

The strong correlation between $\mathrm{M}_{\mathrm{X}}$ (gas) $/\left(\mathrm{M}_{\mathrm{H}_{2}}\right.$ $\left.+\mathrm{M}_{\mathrm{HI}}\right)$ and SFR is consistent with the recent Moreno et al. (2019) simulations of star formation and feedback in galaxy mergers, in which they investigate the relative amounts of hot, warm, cool, and cold-dense gas. In their models, the interaction causes an increase in the amount of cold ultra-dense interstellar gas by a factor of about three on average. This enhances the SFR. The amount of hot gas increases during the starburst (by about 400\%), while the total amount of cold and warm gas mass decreases only slightly or remains constant. The net effect would be an increase in $\mathrm{M}_{\mathrm{X}}$ (gas) $/\left(\mathrm{M}_{\mathrm{H}_{2}}+\mathrm{M}_{\mathrm{HI}}\right)$ during the burst, consistent with our correlation with SFR. In the Moreno et al. (2019) models, the hot gas is produced solely by stellar/supernovae feedback; they do not include AGN feedback or a pre-existing hot halo.

The larger scatter in the $\mathrm{M}_{\mathrm{X}}$ (gas) $/\left(\mathrm{M}_{\mathrm{H}_{2}}+\right.$ $\left.\mathrm{M}_{\mathrm{HI}}\right)$ vs. SFE correlation and its weaker correlation compared to $\mathrm{M}_{\mathrm{X}}$ (gas) $/\left(\mathrm{M}_{\mathrm{H}_{2}}+\mathrm{M}_{\mathrm{HI}}\right)$ vs. SFR is likely due to some low SFR systems having high SFEs; it is the SFR that directly controls the amount of hot gas rather than the SFE.

The weak trend of increasing $\mathrm{M}_{\mathrm{X}}$ (gas) $/\left(\mathrm{M}_{\mathrm{H}_{2}}+\right.$ $\mathrm{M}_{\mathrm{HI}}$ ) with increasing $\mathrm{F}_{60} / \mathrm{F}_{100}$ ratio may be another indirect consequence of the $\mathrm{M}_{\mathrm{X}}$ (gas) $/\left(\mathrm{M}_{\mathrm{H}_{2}}\right.$ $\left.+\mathrm{M}_{\mathrm{HI}}\right)$ vs. SFR correlation. Since $\mathrm{F}_{60} / \mathrm{F}_{100}$ increases with SFR on average for our sample galaxies (Figure 2), galaxies with higher $\mathrm{M}_{\mathrm{X}}(\mathrm{gas}) /\left(\mathrm{M}_{\mathrm{H}_{2}}\right.$ $\left.+\mathrm{M}_{\mathrm{HI}}\right)$ and SFR tend to have larger $\mathrm{F}_{60} / \mathrm{F}_{100}$.

Another factor that may affect $\mathrm{M}_{\mathrm{X}}$ (gas) is escape of hot gas from the gravitational field of the galaxy, particularly in low mass systems. Our 
data shows a hint of lower $\mathrm{M}_{\mathrm{X}}$ (gas)/SFR for low $\mathrm{L}_{\mathrm{K}}$ systems (Figure 13). However, this is uncertain since our sample only includes a few low mass systems. The majority of galaxies in our sample lie in only a small range of $\mathrm{L}_{\mathrm{K}}\left(10^{10} \mathrm{~L}_{\odot}\right.$ $-10^{11.5} \mathrm{~L}_{\odot}$ ), thus it is difficult to find trends with $\mathrm{L}_{\mathrm{K}}$ in our sample. Low mass galaxies may have lower ratios of baryonic mass $\mathrm{M}_{\text {baryon }}$ to dynamical mass $\mathrm{M}_{\mathrm{dyn}}$ compared to high mass systems (e.g., Côté. Carignan, \& Freeman 2000; Torres-Flores et al. 2011). The lower $\mathrm{M}_{\text {baryon }} / \mathrm{M}_{\mathrm{dyn}}$ in low mass systems has been attributed to either mass loss from galactic winds (e.g., van den Bosch 2000; Brook et al. 2012) or less efficient infall into lower mass dark halos (e.g., Sales et al. 2017). A deficiency in hot gas in low mass systems, if confirmed, may point to increased escape of baryons via winds. A larger Chandra imaging survey including more low mass systems would be helpful to better characterize $\mathrm{M}_{\mathrm{X}}$ (gas)/SFR and the scatter in this ratio for low mass galaxies.

To search for additional evidence that the hot gas content in our sample galaxies is affected by the mass and/or the older stellar population in addition to the SFR, we calculated the residuals from the best-fit linear relations for $\log \mathrm{SFR}$ vs. $\log \mathrm{L}_{\mathrm{K}}$, $\log \mathrm{M}_{\mathrm{X}}$ (gas) vs. $\log \mathrm{SFR}$, and $\log \mathrm{M}_{\mathrm{X}}$ (gas) vs. $\log$ $\mathrm{L}_{\mathrm{K}}$. We then searched for correlations between these residuals (top three panels in Figure 19). Strong correlations between these residuals might suggest the existence of a 'fundamental plane' of $\mathrm{M}_{\mathrm{X}}$ (gas) vs. $\log \mathrm{SFR}$ vs. $\log \mathrm{L}_{\mathrm{K}}$.

A weak correlation is seen between the residuals of $\log \mathrm{M}_{\mathrm{X}}$ (gas) vs. SFR and those of $\mathrm{M}_{\mathrm{X}}$ (gas) vs. $\mathrm{L}_{\mathrm{K}}$ (Figure 19, upper right). A strong correlation is seen between the residuals of $\log \mathrm{M}_{\mathrm{X}}$ (gas) vs. $\log \mathrm{L}_{\mathrm{K}}$ and those of $\log \mathrm{SFR}$ vs. $\log \mathrm{L}_{\mathrm{K}}$ (Figure 19, left panel, middle row). The most discrepant galaxies in this plot are NGC 5018, NGC 2865, and Arp 222 (the three stage 7 mergers in the lower left corner). All of these were identified in Paper I as post-starbursts, and all have low sSFR (i.e., have large negative residuals in the log SFR vs. $\log \mathrm{L}_{\mathrm{K}}$ relation). They also have large negative residuals compared to the best-fit $\log \mathrm{M}_{\mathrm{X}}$ (gas) vs. $\log \mathrm{L}_{\mathrm{K}}$ relation. What is discrepant about these galaxies is their $\mathrm{K}$ band luminosities, which are high relative to their SFRs. Figure 19 shows that NGC 1700 has a high relative $\mathrm{M}_{\mathrm{X}}$ (gas) compared to these other low sSFR galaxies.
In the bottom row of Figure 19, we also compared these residuals with $\mathrm{L}_{\mathrm{K}}$ and the SFR. There is a positive correlation between the residuals of the $\mathrm{M}_{\mathrm{X}}$ (gas) vs. $\mathrm{L}_{\mathrm{K}}$ relation, and the SFR (Figure 19, bottom left). Galaxies with low SFRs tend to be deficient in $\mathrm{M}_{\mathrm{X}}$ (gas) compared to the $\mathrm{M}_{\mathrm{X}}$ (gas) vs. $\mathrm{L}_{\mathrm{K}}$ relation. That is because they also tend to have low sSFRs, and it is the SFR that determines the mass of hot gas, not $\mathrm{L}_{\mathrm{K}}$. The galaxies in the lower corner of that plot have low SFRs compared to their $\mathrm{K}$ band luminosities, and therefore they have low $\mathrm{M}_{\mathrm{X}}$ (gas) compared to their $\mathrm{L}_{\mathrm{K}}$.

We also see a positive correlation between the residuals of the $\mathrm{M}_{\mathrm{X}}$ (gas) vs. SFR relation, and $\mathrm{L}_{\mathrm{K}}$ (Figure 19, bottom right panel). NGC 1700 stands out as having excess hot gas, while systems with low $\mathrm{K}$ band luminosities tend to have less hot gas relative to the $\mathrm{M}_{\mathrm{X}}$ (gas) vs. SFR relation. This suggests that some gas may have been lost from these systems. Unfortunately, our sample only contains a few galaxies with low $\mathrm{K}$ band luminosities, and only a few galaxies with low sSFR, so this result is uncertain.

We conclude that only a few galaxies in our sample deviate from a straight line in the log $\mathrm{M}_{\mathrm{X}}$ (gas) $-\log \mathrm{SFR}-\log \mathrm{L}_{\mathrm{K}}$ plane. To better understand these deviations, it would be helpful to increase the number of post-starburst galaxies in our sample, as well as the number of low mass galaxies.

\subsection{Trends with $\mathrm{M}_{\mathrm{X}}$ (gas)/SFR and $\mathbf{n}_{\mathrm{e}} \sqrt{f}$}

In addition to the strong positive correlations discussed above, some weak anti-correlations are also seen in the data:

(1) There are weak anti-correlations between $\mathrm{M}_{\mathrm{X}}$ (gas)/SFR and $\mathrm{F}_{60} / \mathrm{F}_{100}$, and between $\mathrm{M}_{\mathrm{X}}$ (gas)/SFR and [3.6] - [24] (Figure 14). These anti-correlations hold even when low SFR systems are excluded.

(2) As $M_{X} / S F R$ goes up, $n_{e} \sqrt{f}$ goes down, even when low SFR systems are excluded (Figure 14). This is also a weak trend.

(3) There is a weak trend of decreasing volume with increasing $n_{e} \sqrt{f}$ (Figure 11).

Some parameters are neither correlated or anti-correlated: 
(1) $M_{X}($ gas)/SFR is not correlated with SFR, if low SFR systems are excluded (Figure 13). $\mathrm{M}_{\mathrm{X}}$ (gas)/SFR is not correlated with for a variable $\mathrm{CO} / \mathrm{H}_{2}$ ratio (Figure 14).

(2) The SFR, [3.6] - [24], and $\mathrm{F}_{60} / \mathrm{F}_{100}$ do not correlate with $\mathrm{n}_{\mathrm{e}} \sqrt{f}$ (Figure 12).

(3) No significant correlations are found between $\mathrm{M}_{\mathrm{X}}$ (gas)/SFR and volume (Table 5), and no correlation between $\mathrm{M}_{\mathrm{X}}$ (gas)/SFR and $\mathrm{L}_{\mathrm{FIR}} / \mathrm{L}_{\mathrm{K}}$ when low SFR systems are excluded (Figure 14).

Although $\mathrm{M}_{\mathrm{X}}$ (gas)/SFR is anti-correlated with $\mathrm{F}_{60} / \mathrm{F}_{100}$ and with [3.6] - 24], $\mathrm{M}_{\mathrm{X}}(\mathrm{gas}) / \mathrm{SFR}$ is not correlated (either positively or negatively) with SFR, in spite of the fact that $\mathrm{F}_{60} / \mathrm{F}_{100}$ and [3.6] - [24] are both (weakly) correlated with SFR. Furthermore, although volume and $\mathrm{n}_{\mathrm{e}} \sqrt{f}$ are weakly anti-correlated, and SFR is correlated with volume, $\mathrm{n}_{\mathrm{e}} \sqrt{f}$ is not correlated with SFR.

These results suggest that another factor contributes to the observed variations in $\mathrm{M}_{\mathrm{X}}$ (gas)/SFR and $n_{e} \sqrt{f}$ besides SFR. One possibility is differences in timescale; variations in the age of an on-going starburst or the time since the end of a starburst may affect $\mathrm{n}_{\mathrm{e}} \sqrt{f}$ and $\mathrm{M}_{\mathrm{X}}$ (gas)/SFR, as well as other parameters of the system. Numerical simulations show that interaction-triggered starbursts can last for extended periods $(\geq 100$ Myrs; Lotz et al. 2000; Di Matteo et al. 2008; Bournaud et al. 2011; Fensch et al. 2017). This timescale is similar to the radiative cooling times for the gas (median of 60 Myrs, see Section 5); it is also similar to the timescale over which the UV data is measuring the SFR $(\sim 100$ Myrs; Kennicutt \& Evans 2012). If the cooling time is less than the timescale over which the SFR is measured, and if the cooling time is less than the age of the burst, then late in a burst the $\mathrm{M}_{\mathrm{X}}$ (gas)/SFR may decrease (i.e., some hot gas has cooled, but the UV-bright stars contributing to our SFR estimate have not yet died). The sSFR as measured by [3.6] - [24] and $\mathrm{L}_{\mathrm{FIR}} / \mathrm{L}_{\mathrm{K}}$ may also vary with time during a burst. Presumably the electron density and/or filling factor also evolve with time during a burst, along with $\mathrm{F}_{60} / \mathrm{F}_{100}$, the volume of hot gas, and $\mathrm{M}_{\mathrm{X}}$ (gas)/SFR. Further theoretical modeling is needed to better understand the relationships between these parameters in evolving starbursts.

A second factor that may contribute to variations in $\mathrm{M}_{\mathrm{X}}$ (gas)/SFR and $\mathrm{n}_{\mathrm{e}} \sqrt{f}$ may be the efficiency of early feedback. According to numerical simulations, stellar winds and radiation pressure early in a starburst disrupt molecular clouds, making it easier for subsequent supernovae to produce hot gas (Hopkins et al. 2012a; Agertz et al. 2013; Hopkins et al. 2013b). The efficiency of early feedback might be related to the spatial density of star formation; more concentrated distributions of young stars may have more early UV radiation per volume, allowing quicker destruction of molecular gas. This may lead to easier escape for hot gas from the region, and thus less diffuse Xray emission. More concentrated distributions of young stars would presumably lead to more intense UV interstellar radiation fields and therefore hotter dust and higher $\mathrm{F}_{60} / \mathrm{F}_{100}$ ratios (e.g., Desert, Boulanger, \& Puget 1990). The $\mathrm{F}_{60} / \mathrm{F}_{100}$ ratio is weakly anti-correlated with $\mathrm{M}_{\mathrm{X}}$ (gas)/SFR, consistent with this scenario. The [3.6] - [24] color may also increase with higher spatial concentrations of young stars, and [3.6] - [24] is also weakly anti-correlated with $\mathrm{M}_{\mathrm{X}}$ (gas)/SFR. Further study is needed to investigate how all of these parameters vary with the density of OB stars in a galaxy.

A third factor that might affect $\mathrm{M}_{\mathrm{X}}$ (gas)/SFR is the initial mass function (IMF). A top-heavy IMF may lead to an increase in supernovae compared to lower mass stars, which might produce a larger $\mathrm{M}_{\mathrm{X}}$ (gas)/SFR when the SFR is derived from the UV continuum. It has been suggested that high SFR and/or high SFE galaxies may have IMFs skewed to high mass stars (Rieke et al. 1980; Elbaz. Arnaud, \& Vangioni-Flam 1995; Brassington et al. 2007; Köppen, Weidner, \& Kroupa 2007; Weidner et al. 2013; Brown \& Wilson 2019). Thus one might expect higher $\mathrm{M}_{\mathrm{X}}$ (gas)/SFR for higher $\mathrm{SFR}$ or higher SFE systems. However, we do not see a correlation between $\mathrm{M}_{\mathrm{X}}$ (gas)/SFR and SFR, or between $M_{X}$ (gas)/SFR and SFE. This means that either IMF variations are not responsible for the spread in $\mathrm{M}_{\mathrm{X}}$ (gas)/SFR, or the IMF is not correlated with SFR or SFE.

Another factor that might affect $\mathrm{M}_{\mathrm{X}}$ (gas)/SFR and $n_{e} \sqrt{f}$ is metallicity. A number of studies have concluded that the SFR of star-forming galaxies depends upon metallicity in addition to stellar mass; for the same stellar mass, lower metallicity 
systems have higher SFRs (Ellison et al. 2008; Mannucci et al. 2010; Lara-López et al. 2010; Hirschauer et al. 2018, but see Izotov et al. 2014, 2015). This result has been explained by infall of low metallicity gas, fueling star formation. Our $\mathrm{M}_{\mathrm{X}}$ (gas)/SFR values may be artificially skewed by metallicity, since the value of $\mathrm{L}_{\mathrm{X}}$ (gas) that is derived from the Chandra spectra is affected by metallicity (see Paper I). In addition, the fraction of the supernovae and stellar wind energy converted into X-ray flux may be a function of metallicity. A larger sample of galaxies including more low metallicity systems would be helpful to investigate this issue further.

Unfortunately, we do not have a measure of the volume filling factor of the hot gas, f, independently of $n_{e}$, to determine whether $f$ varies significantly from system to system. Based on theoretical arguments and/or hydrodynamical simulations, for a range of systems f has variously been estimated to be 70-80\% (McKee \& Cowie 1977), 20-40\% (Breitschwerdt et al. 2012), 30-40\% (Kim \& Ostriker 2017), or anywhere between 10-90\% depending upon the supernovae rate and the average gas density (Li et al. 2015). In general, according to simulations the higher the density of star formation, the larger the expected hot gas filling factor (Breitschwerdt et al. 2012; Li et al. 2015). One might expect higher SFRs to produce faster winds, as has been found for the warm ionized medium (e.g., Heckman et al. 2015). A faster wind may lead to lower $n_{e}$ values. If the filling factor increases with SFR but $\mathrm{n}_{\mathrm{e}}$ decreases, this might explain the lack of a trend between $\mathrm{n}_{\mathrm{e}} \sqrt{f}$ and SFR. Independent determinations of $\mathrm{n}_{\mathrm{e}}$ and f (e.g., Kregenow et al. 2006; Jo et al. 2019) are needed to test this possibility.

\subsection{The Scatter in $M_{X}$ (gas)}

One of the major conclusions of the current paper is that, excluding low SFR systems, $\mathrm{M}_{\mathrm{X}}$ (gas)/SFR is constant with SFR with an rms spread of only 0.34 dex. A number of factors may contribute to this scatter, in addition to age, metallicity, or IMF differences. First, as discussed in Section 5, the decomposition of the X-ray spectrum into a thermal and a non-thermal component introduces some uncertainty, adding uncertainty to our determination of $\mathrm{L}_{\mathrm{X}}$ (gas) (see Paper I). Second, as also discussed in Section 5, the un- known extent of the hot gas along our line of sight leads to uncertainties in the volume of the hot gas, which contributes to the scatter in the parameters derived from the volume. Systematic variations in the geometry of the hot gas may further affect the observed relations. For example, systems with lower SFR may have disk-like distributions of cold gas, with coronal gas extending out of the galactic plane, while higher SFR systems, which are more likely to be in the midst of a merger, may have gas distributions which are more spherical. Another factor that may contribute to the scatter are system-to-system variations in the gravitational masses of the galaxies, which likely affect outflow rates and potential loss of hot gas. We found that galaxies with low $\mathrm{K}$ band luminosities tend to have lower $\mathrm{M}_{\mathrm{X}}$ (gas)/SFR ratios compared to other galaxies (Figure 16), suggesting that low mass galaxies may lose some hot gas. The large-scale environment may also affect the $\mathrm{M}_{\mathrm{X}}$ (gas)/SFR ratio, however, $\mathrm{L}_{\mathrm{X}}$ (gas)/SFR is not correlated with local galaxy density $(\mathrm{Pa}-$ per I); $\mathrm{M}_{\mathrm{X}}$ (gas)/SFR and $\mathrm{M}_{\mathrm{X}}$ (gas) $/\left(\mathrm{M}_{\mathrm{H}_{2}}+\mathrm{M}_{H I}\right)$ are also not correlated with local galaxy density. Another factor that may contribute to the observed scatter in these plots is our assumption of a temperature of $\mathrm{kT}=0.3 \mathrm{keV}$ for the hot gas in the systems without an X-ray determination of temperature. Longer Chandra exposures would be useful to spectroscopically determine the temperature of the gas in more of the galaxies.

\subsection{NGC 1700}

As noted several times in this paper, the latestage merger NGC 1700 does not fit some of the strong relations seen in this study. NGC 1700 has a large X-ray size relative to its SFR. It also has a high X-ray luminosity and a large mass of hot X-ray-emitting gas. This suggests that either NGC 1700 is in a special evolutionary state compared to the other systems in our sample, or it acquired its hot gas via a different process. Maybe NGC 1700 was a pre-existing elliptical that already had a large amount of hot gas, which then swallowed a gas-rich galaxy. It is sometimes difficult to distinguish between the remnant of a spiral-spiral merger and the remnant of an elliptical-spiral merger. In appearance, NGC 1700 is an elliptical-like galaxy surrounded by tidal debris, but its merger history is uncertain. 
It was classified as the remnant of a spiral-spiral major merger by Schweizer \& Seitzer (1992) and Brown et al. (2000), however, Statler et al. (1996) and Kleineberg, Sánchez-Blázquez, \& Vazdekis (2011) conclude that it is the result of the merger of at least three galaxies, two large spirals and a third smaller galaxy. If NGC 1700 is the product of a single major merger, perhaps it is in a later stage in the conversion from a major merger to an elliptical than the other post-merger galaxies in our sample. Theory suggests that ellipticals produced by major mergers can build a large quantity of hot gas by the virialization of gas lost from red giants in the gravitational potential well, with possible heating by Type Ia supernovae and/or AGN feedback (Ciotti et al. 1991, 2017; Pellegrini \& Ciotti 1998; Mathews \& Brighenti 2003). This process is expected to be very slow, with timescales of many gigayears. Expanding our sample to include more galaxies like NGC 1700 would be helpful to better understand how hot gas grows in such systems. More generally, increasing the number of low sSFR galaxies in our sample is needed to investigate how the hot gas in galaxies evolves as star formation fades in a quenched or quenching galaxy.

\section{Summary}

We have measured the spatial extent of the hot interstellar gas in a sample of 49 interacting and merging galaxies in the nearby Universe. For systems with $\mathrm{SFR}>1 \mathrm{M}_{\odot}$ yr, we found strong nearlinear correlations between the volume of hot gas and the SFR, and between $\mathrm{M}_{\mathrm{X}}$ (gas) and SFR. This supports the idea that supernovae and stellar winds are responsible for the hot gas. As expected, the $\mathrm{M}_{\mathrm{X}}$ (gas) $/\left(\mathrm{M}_{\mathrm{H}_{2}}+\mathrm{M}_{\mathrm{HI}}\right)$ ratio also increases linearly with increasing SFR for high SFR systems. These results are consistent with recent hydrodynamical simulations of interactions including feedback. The $\mathrm{M}_{\mathrm{X}}$ (gas) $/\left(\mathrm{M}_{\mathrm{H}_{2}}+\mathrm{M}_{\mathrm{HI}}\right)$ ratio also increases with dust temperature on average, perhaps due to a larger proportion of dust associated with the hot gas.

In low SFR, low sSFR systems, we find evidence for an excess of hot gas relative to the relations for higher SFR systems. This excess may be associated with mass loss from older stars. However, our sample only includes a few galaxies with low
sSFR rates, so this result is uncertain. In addition, we see a possible deficient of hot gas in low mass systems, perhaps due to escape from the gravitational field of the galaxy. However, this result is also uncertain due to the small number of low mass systems in our sample.

The $\mathrm{M}_{\mathrm{X}}$ (gas)/SFR is weakly anti-correlated with $\mathrm{F}_{60} / \mathrm{F}_{100}$, [3.6] - [24], and $\mathrm{n}_{\mathrm{e}} \sqrt{f}$. The inferred electron density decreases with increasing volume of hot gas assuming a constant filling factor. These results may be a consequence of variations in the spatial density of young stars, age of the stars, metallicity, IMF, and/or efficiency of feedback in these galaxies.

This research was supported by NASA Chandra archive grant AR6-17009X, issued by the Chandra X-ray Observatory Center, which is operated by the Smithsonian Astrophysical Observatory for and on behalf of NASA under contract NAS8-03060. Support was also provided by National Science Foundation Extragalactic Astronomy Grant ASTR-1714491. The scientific results reported in this article are based on data obtained from the Chandra Data Archive. This research has also made use of the NASA/IPAC Extragalatic Database (NED), which is operated by the Jet Propulsion Laboratory, California Institute of Technology, under contract with NASA. This work also utilizes archival data from the Spitzer Space Telescope, which is operated by the Jet Propulsion Laboratory (JPL), California Institute of Technology under a contract with NASA. This study also uses archival data from the NASA Galaxy Evolution Explorer (GALEX), which was operated for NASA by the California Institute of Technology under NASA contract NAS5-98034. 


\section{APPENDIX: Chandra $0.3-1.0 \mathrm{keV}$ Maps}

For the 44 systems for which we can measure X-ray radial profiles, the unsmoothed Chandra $0.3-1.0$ $\mathrm{keV}$ maps are displayed in the right panels of Figures $20-27$. When only one Chandra dataset is available for the galaxy, the ciao command fluximage was used to convert into units of photons s ${ }^{-1} \mathrm{~cm}^{-2} \operatorname{arcsec}^{-2}$, using an exposure-correction map with a $0.8 \mathrm{keV}$ effective energy. When multiple Chandra datasets are available for one system, the datasets have been merged together using the ciao command merge_obs, which also does the exposure correction and flux calibration. The left panels of Figures $20-27$ show either the SDSS g band image (when available) or the GALEX NUV image. Contours of the X-ray surface brightness are overlaid on the Chandra images. These have been lightly smoothed using the ds9 softwar 7 , with the smooth parameter set to 6 .

${ }^{7}$ SAOImageDS9 development has been made possible by funding from the Chandra X-ray Science Center (CXC) and the High Energy Astrophysics Science Archive Center (HEASARC) with additional funding from the JWST Mission office at Space Telescope Science Institute. 


\section{REFERENCES}

Agertz, O. \& Kravtsov, A. V. 2015, ApJ, 804, 18

Agertz, O. \& Kravtsov, A. V. 2016, ApJ, 824, 79

Agertz, O., Kravtsov, A. V., Leitner, S. N., \& Gnedin, N. Y. 2013, ApJ, 770, 25

Andreani, P., Boselli, A., Ciesla, L., Vio, R., Cortese, L., Buat, V., \& Miyamoto, Y. 2018, A\&A, 617, 33

Arp, H. C. 1966, Atlas of Peculiar Galaxies (Pasadena, CA: Caltech)

Balick, B. \& Heckman, T. 1981, A\&A, 96, 271

Bell, E. F. \& de Jong, R. S. 2000, ApJ, 550, 212

Bolatto, A. D., Wolfire, M., \& Leroy, A. K. 2013, ARAA, 51, 207

Boroson, B., Kim, D.-W., \& Fabbiano, G. 2011, ApJ, 729, 12

Bournaud, F., Chapon, D., Teyssier, R. et al. 2011, ApJ, 730, 4.

Brassington, N. J., Ponman, T. J., \& Read, A. M. 2007, MNRAS, 337, 1439

Breitschwerdt, D., de Avillez, M. A., Feige, J., \& Dettbarn, C. 2012, AN, 333, 486

Brinchmann, J., Charlot, S., White, S. D. M., et al. 2004, MNRAS, 351, 1151

Brook, C. B., Stinson, G., Gibson, B. K., Wadsley, J., \& Quinn, T. 2012, MNRAS, 424, 1275

Brown, R. J. N., Forbes, D. A., Kissler-Patig, M., \& Brodie, J. P. 2000, MNRAS, 317, 406

Brown, T. \& Wilson, C, 2019, ApJ, in press (arXiv: 1905.06950).

Bushouse, H. A. 1987, ApJ, 320, 49

Bushouse, H. A., Lord, S. D., Lamb, S. A., Werner, M. W., \& Lo, K. Y. 1999, astro-ph/9911186

Bustard, C., Zweibel, E. G., \& D'Onghia, E. 2016, ApJ, 819, 29

Casoli, F., Dupraz, C., Combes, F., \& Kazes, I. 1991 A\&A, 251, 1
Chevalier, R. A. \& Clegg, A. W. 1985, Nature, 317,44

Ciotti, L., D'Ecole, A., Pellegrini, S., \& Renzini, A. 1991, ApJ, 376, 380

Ciotti, L., Pellegrini, S., Negri, A., \& Ostriker, J. P. 2017, ApJ, 835, 15

Côté, S., Carignan, C., \& Freeman, K. C. 2000, AJ, 120,3027

Cox, A. L. \& Sparke, L. S. 2004, AJ, 128, 2013

Cox, T. J., Dutta, S. N., Di Matteo, T., et al. 2006a, ApJ, 650, 791

Cox, T. J., Di Matteo, T, Hernquist, L, et al. 2006b, ApJ, 643, 692

Cox, T. J., Jonsson, P., Primack, J. R., \& Somerville, R. 2006c, MNRAS, 373, 1013

Daddi, E., Elbaz, D., Walter, F., et al. 2010, ApJ, 714,418

Dame, T. M., Hartmann, D., \& Thaddeus, P. 2001, ApJ, 547, 792

Desert, F.-X., Boulanger, F., \& Puget, J. L. 1990, A\&A, 237, 215

Di Matteo, P., Bournaud, F., Martig, M., Combes, F., Melchior, A. -L., \& Semelin, B. 2008, A\&A, 492,31 .

Downes, D. \& Solomon, P. M. 1998, ApJ, 507, 615

Doyle, M. T., Drinkwater, M. J., Rohde, D. J., et al. 2005, MNRAS, 361, 34

Elbaz, D., Arnaud, M., \& Vangioni-Flam, E. 1995, A\&A, 303, 345

Ellison, S. L., Patton, D. R., Simard, L., \& McConnachie, A. W. 2008, ApJ, 672, L107

Elmegreen, B. G., Kaufman, M., Bournaud, F., et al. 2016, ApJ, 823, 26

English, J., Norris, R. P., Freeman, K. C., \& Booth, R. S. 2003, AJ, 125, 1134

Fensch, J., Renaud, F., Bournaud, F., et al. 2017, MNRAS, 465, 1934

Fernández, X., Petric, A. O., Schweizer, F., \& van Gorkom, J. H. 2014, AJ, 147, 74 
Finlator, K. \& Davé, R. 2008, MNRAS, 385, 2181

Goulding, A., Greene, J. E., Ma, C.-P., et al. 2016, ApJ, 826, 167

Gao, Y. \& Solomon, P. M. 2004, ApJS, 152, 63

Gaspari, M., Eckert, D., Ettori, S., et al. 2019, astro-ph/1904.10972

Georgakakis, A., Forbes, D. A., \& Norris, R. P. 2000, MNRAS, 318, 124

Georgakakis, A., Hopkins, A. M., Caulton, A., Wiklind, T., Terlevich, A. I., \& Forbes, D. A. 2001, MNRAS, 326, 1431

Gordon, S., Koribalski, B., \& Jones, K. 2001, MNRAS, 326, 578

Grimes, J. P., Heckman, T., Strickland, D., \& Ptak, A. 2005, ApJ, 628, 187

Hayward, C. C., Torrey, P., Springel, V., Hernquist, L., \& Vogelsberger, M. 2014, MNRAS, 442, 1992

Heckman, T. M., Alexandroff, R. M., Borthakur, S., Overzier, R., \& Leitherer, C. 2015, ApJ, 809, 147

Hibbard, J. E. \& van Gorkom, J., 1996, AJ, 111, 655

Hirschauer, A. S., Salzer, J. J., Janowiecki, S., \& Wegner, G. A. 2018, AJ, 155, 82

Hopkins, P. F., Quataert, E., \& Murray, N. 2011, MNRAS, 417, 950

Hopkins, P. F., Quataert, E., \& Murray, N. 2012b, MNRAS, 421, 3488

Hopkins, P. F., Quataert, E., \& Murray, N. 2012a, MNRAS, 421, 3522

Hopkins, P. F., Kereš, D., Norman, M., Hernquist, L., Narayanan, D., \& Hayward, C. C. 2013, MNRAS, 433, 78

Hopkins, P., Kereš, D., Önorbe, J. 2014, MNRAS, 445,581

Hopkins, P. F., Narayanan, D., Murray, N., \& Quataert, E. 2013b, MNRAS, 433, 69
Horellou, C., Casoli, F., Combes, F., \& Dupraz, C. 1995, A\&A, 298, 743

Houck, J. C. \& Denicola, L. A. 2000, Astronomical Data Analysis Software and Systems IX, ASP Conference Proceedings, Vol. 216, ed. N. Manset, C. Veillet, \& D. Crabtree, Astronomical Society of the Pacific, p. 591

Huchtmeier, W. K. \& Richter, O.-G. 1989, A General Catalog of HI Observations of Galaxies (New York: Springer-Verlag)

Into, T. \& Portinari, L. 2013, MNRAS, 430, 2715

Inami, H., Armus, L., Charmandaris, V., et al. 2013, ApJ, 777, 156

Israel, F. P. 2005, A\&A, 438, 8551

Izotov, Y. I., Guseva, N. G., Fricke, K. J., \& Henkel, C. 2014, A\&A, 561, A33

Izotov, Y. I., Guseva, N. G., Fricke, K. J., \& Henkel, C. 2015, MNRAS, 451, 2251

Jester, S., Schneider, D. P., Richards, G. T., et al. 2005, AJ, 130, 873

Jo, Y.-S., Seon, K., Min, K.-W., Edelstein, J., \& Han, W. 2019, ApJS, arXiv:1905.07823

Juneau, S., Narayanan, D. T., Moustakas, J., Shirley, Y. L., Bussmann, R. S., Kennicutt, R. C., Jr., \& Vander Bout, P. A. 2009, ApJ, 707, 1217

Kennicutt, R. C., Jr. 1998, ARAA, 36, 189

Kennicutt, R. C. \& Evans, N. J. 2012, ARAA, 50, 531

Kennicutt, R. C., Jr., Hao, C.-N., Calzetti, D., et al. 2009, ApJ, 703, 1672

Kereš, D., Katz, N., Davé, R., Fardal, M., \& Weinberg, D. H. 2009, MNRAS, 396, 2332

Kim, D.-W. \& Fabbiano, G. 2013, ApJ, 776, 116

Kim, C.-G. \& Ostriker, E. C. 2017, ApJ, 846, 133

Kleineberg, K., Sánchez-Blázquez, P., \& Vazdekis, A. 2011, ApJ, 732, 33

Köppen, J., Weidner, C., \& Kroupa, P. 2007, MNRAS, 375,673 
Koski, A. T. 1978, ApJ, 223, 56

Kotilainen, J. K., Moorwood, A. F. M., Ward, M. J., \& Forbes, D. A. 1996, A\&A, 305, 107

Kregenow, J. M., Sirk, M., Sankrit, R., et al. 2006, AAS, 209, 1702

Lara-López, M. A., Cepa, J., Bongiovanni, A., et al. 2010, A\&A, 521, 53

Larson, K., Sanders, D. B., Barnes, J. E., et al. 2016, ApJ, 825, 128

Li, J.-T. \& Wang, Q. D. 2013, MNRAS, 435, 3071

Li, M., Ostriker, J. P., Cen, R., Bryan, G. L., \& Naab, T. 2015, ApJ, 814, 4

Lotz, J. M., Jonsson, P., Cox, T. J., \& Primack, J. R. 2000, MNRAS, 391, 1137

Ma, X., Hopkins, P. F., Faucher-Giguere, C.-A., et al. 2016, MNRAS, 456, 2140

Mannucci, F., Cresci, G., Maiolini, R., Marconi, A., \& Gnerucci, A. 2010, MNRAS, 408, 2115

Martin, J. M., Bottinelli, L., Dennefeld, M., \& Gouguenheim, L. 1991, A\&A, 245, 393

Maraston, C. 1998, MNRAS, 300, 872

Mathews, W. G. \& Brighenti, F. 2003, ARA\&A, 41, 191

McCray, R. 1987, in Spectroscopy of Astrophysical Plasmas, ed. A. Dalgarno \& D. Layzer (Cambridge: Cambridge Univ. Press), 260

McKee, C. F. \& Cowie, L. L. 1977, ApJ, 215, 213

McQuinn, K. B. W., Skillman, E. D., Heilman, T. N., Mitchell, N. P., \& Kelley, T. 2018, 477, 3164

Meiksin, A. 2016, MNRAS, 461, 2762

Mineo, S., Gilfanov, M., \& Sunyaev, R. 2012b, MNRAS, 426, 1870

Mirabel, I. F., Booth, R. S., Garay, G., Johansson, L. E. B., \& Sanders, D. B. 1990, A\&A, 236, 327

Moreno, J., Torrey, P., Ellison, S. L., et al. 2019, MNRAS, 485, 1320

Muratov, A. L., Kereš, D., Faucher-Giguère, C.A., et al. 2015, MNRAS, 454, 2691
Noeske, K. G., Weiner, B. J., Faber, S. M., et al. 2007, ApJ, 660, L43

Obreschkow, D. \& Rawlings, S. 2009, MNRAS, 394,1857

Owen, R. A. \& Warwick, R. S. 2009, MNRAS, 394, 1741

Orr, M. E., Hayward, C. C., Hopkins, P. F., et al. 2018, MNRAS, 478, 3653

O'Sullivan, E., Forbes, D. A., \& Ponman, T. J. 2001, MNRAS, 328, 461

Papadopoulos, P. P., van der Werf, P. P., Xilouris, E. M., et al. 2012, MNRAS, 426, 2601

Pellegrini, S. \& Ciotti, L. 1998, A\&A, 333, 433

Renaud, F., Bournaud, F., Agertz, O., et al. 2019, $\mathrm{A} \& \mathrm{~A}, 625,65$

Renaud, F. Bournaud, F., Emsellem, E., et al. 2013, MNRAS, 436, 1836

Renaud, F., Bournaud, F., Emsellem, E. et al. 2015, MNRAS, 454, 3299

Renaud, F., Bournaud, F., Kraljic, K., \& Duc, P.-A. 2014, MNRAS, 442, L33

Rieke, G. H., Lebofsky, M. J., Thompson, R. I., Low, F. J., \& Tokunaga, A. T. 1980, ApJ, 238, 24

Sage, L. J., Salzer, J. J., Loose, H.-H., \& Henkel, C. 1992, A\&A, 265, 19

Sales, L. V., Navarro, J. F., Oman, K., et al. 2017, MNRAS, 464, 2419

Salim, S., Rich, R. M., Charlot, S., et al. 2007, ApJS, 173, 267

Sanders, D. B. \& Mirabel, I. F. 1996, ARAA, 34, 749

Sanders, D. B., Scoville, N. Z., \& Soifer, B. T. 1991, ApJ, 370, 158

Schweizer, F. \& Seitzer, P. 1992, AJ, 104, 1039

Schmidt, M. 1959, ApJ, 129, 243

Smith, B. J., Kleinmann, S. G., Huchra, J. P., \& Low, F. J. 1987, ApJ, 318, 161 
Smith, B. J., Campbell, K., Struck, C., et al. 2018, AJ, 155, 81

Smith, B. J., Struck, C., Hancock, M., Appleton, P. N., Charmandaris, V., \& Reach, W. T. 2007, AJ, 133, 791

Smith, B. J., Swartz, D. A., Miller, O., et al. 2012, AJ, 142, 144

Smith, D. A., Herter, T., Haynes, M. P., Beichman, C. A., \& Gautier, T. N. 1996, ApJS, 104, 217

Soifer, B. T., Sanders, D. B., Madore, B. F., et al. 1987, ApJ, 320, 238

Solomon, P. M., Downes, D., \& Radford, S. J. E. 1992, ApJ, 387, L55

Solomon, P. M., Downes, D., Radford, S. J. E., \& Barrett, J. W. 1997, ApJ, 478, 144

Sparre, M. \& Springel, V. 2016a, MNRAS, 462, 2418

Springel, V. 2000, MNRAS, 312, 859

Statler, T. S., Smecker-Hane, T., \& Cecil, G. N. 1996, AJ, 111, 1512

Strickland, D. K., Heckman, T. M., Weaver, K. A., \& Dahlem, M. 2000, AJ, 120, 2965

Strickland, D. K., Heckman, T. M., Colbert, E. J. M., Hoopes, C. G., \& Weaver, K. A. 2004a, ApJS, 151, 193

Strickland, D. K., Heckman, T. M., Colbert, E. J. M., Hoopes, C. G., \& Weaver, K. A. 2004b, ApJ, 606, 829

Strickland, D. K. \& Stevens, I. R. 2000, MNRAS, 314,511

Su, Y., Irwin, J. A., White, R. E., \& Cooper, M. C. 2015, ApJ, 806, 156

Teyssier, R., Chapon, D., \& Bournaud, F. 2010, ApJ, 720, L149

Torres-Flores, S., Epinat, B., Amram, P., Plana, H., \& Mendes de Olivera, C. 2011, MNRAS, 416, 1936

Torrey, P., Vogelberger, M., Marinacci, F., et al. 2019, MNRAS, 484, 5587
Trujillo-Gomez, S., Klypin, A., Colín, P., Ceverino, D., Arraki, K. S., \& Primack, J. 2015, MNRAS, 446, 1140

Ueda, J., Iono, D., Yun, M. S., et al. 2014, ApJS, 214, 29

van de Voort, F., Quataert, E., Hopkins, P. F., et al. 2016, MNRAS, 463, 4533

van den Bosch, F. C. 2000, ApJ, 530, 177

van Driel, W., Arnaboldi, M., Combes, F., \& Sparke, L. S. 2000, A\&AS, 141, 385

Weider, C., Ferraras, I., Vazdekis, A., \& La Barbera, F. 2013, MNRAS, 435, 2274

Wiklind, T., Combes, F., \& Henkel, C. 1995, A\&A, 297, 643

Wu, J., Evans, N. J., II., Shirley, Y. L., \& Knez, C. 2010, ApJS, 188, 313

Young, J. S., Allen, L., Kenney, J. D. P., Lesser, A., \& Rownd, B. 1996, AJ, 112, 1903

Young, J. S., Kenney, J. D., Tacconi, L., Claussen, M. J., Huang, Y.-L., \& Tacconi-Garman, L. 1986, ApJ, 311, L17

Zhang, D., Thompson, T. A., Murray, N., \& Quataert, E. 2014, ApJ, 784, 93

Zhu, M., Seaquist, E. R., Davoust, E., Frayer, D. T., \& Bushouse, H. A. 1999, AJ, 118, 145

This 2-column preprint was prepared with the AAS LATEX macros v5.2. 

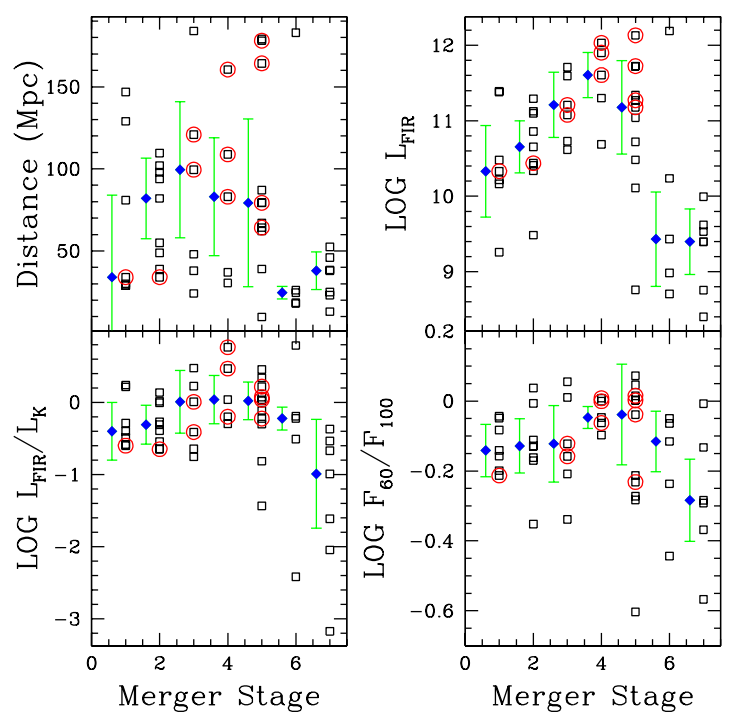

Fig. 1.- Plots of basic galaxy properties (distance, $\mathrm{L}_{\mathrm{FIR}}, \mathrm{L}_{\mathrm{FIR}} / \mathrm{L}_{\mathrm{K}}$, and $\left.\mathrm{F}_{60} / \mathrm{F}_{100}\right)$ vs. merger stage. Black open squares mark the sample galaxies, with those circled by red circles being AGN. The filled blue diamonds are the median values for each stage, slightly offset to the left. The errorbars plotted on the median values are the semiinterquartile range, equal to half the difference between the 75 th percentile and the 25 th percentile.

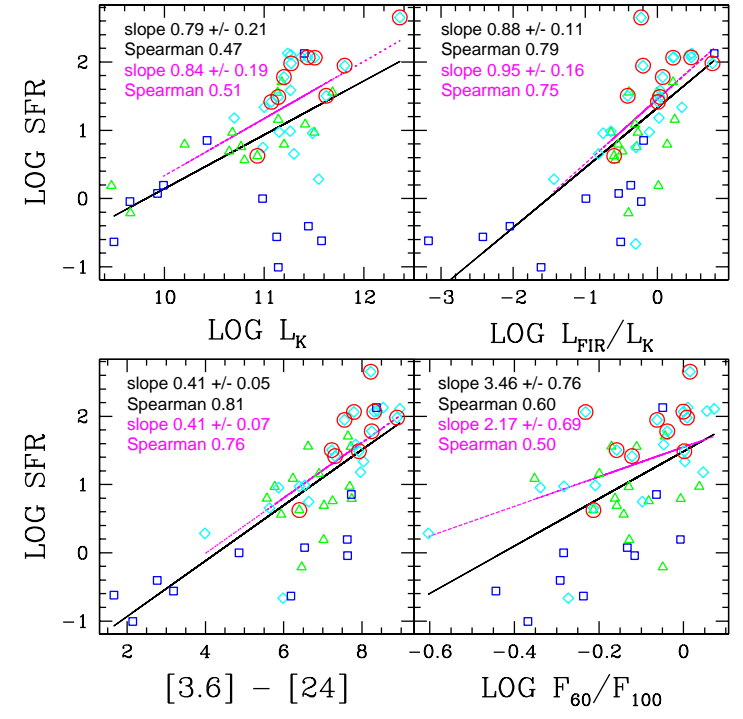

Fig. 2.- Correlations between basic galaxy properties (SFR vs. $\mathrm{L}_{\mathrm{K}}, \mathrm{L}_{\mathrm{FIR}} / \mathrm{L}_{\mathrm{K}},[3.6]-[24]$, and $\left.\mathrm{F}_{60} / \mathrm{F}_{100}\right)$. The best-fit line for the full sample is plotted as a solid black line, while the bestfit line for systems with SFR $>1.0 \mathrm{M}_{\odot} \mathrm{yr}^{-1}$ is given as a dotted line. The best-fit slope and the Spearman rank correlation coefficient for the full set is shown in black (on top), while the values for the high SFR subset are shown in magenta (below). Merger stages 1 and 2 systems are marked as open green triangles. Merger stages 3, 4, and 5 are open cyan diamonds, and merger stages 6 and 7 are identified by blue open squares. AGN are identified by red circles. 


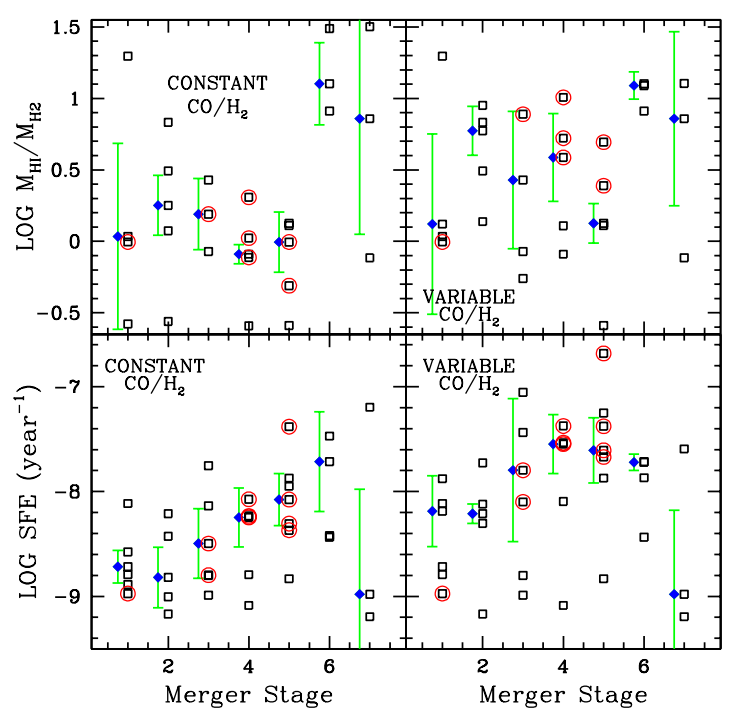

Fig. 3.- Top row: plots of the HI mass $/ \mathrm{H}_{2}$ mass against merger stage. Bottom row: plots of the SFE against merger stage. In the left panels, the standard Galactic $\mathrm{CO} / \mathrm{H}_{2}$ ratio is used for all galaxies. In the right panels, a variable $\mathrm{CO} / \mathrm{H}_{2}$ ratio is used. Black open squares mark the sample galaxies, with those circled by red circles being AGN. The filled blue diamonds are the median values for each stage, slightly offset to the left. The errorbars plotted on the median values are the semi-interquartile range, equal to half the difference between the 75 th percentile and the 25 th percentile.
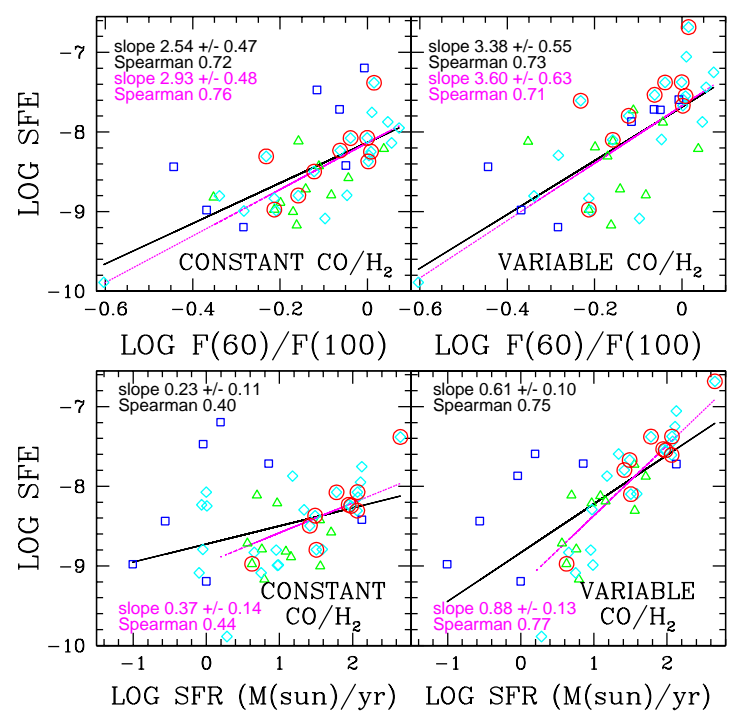

Fig. 4.- Comparisons between $\mathrm{SFE}$ and $\mathrm{F}_{60} / \mathrm{F}_{100}$ (top panels) and SFE and SFR (bottom panels). The left panels use a constant $\mathrm{CO} / \mathrm{H}_{2}$ ratio, while the right use a variable $\mathrm{CO} / \mathrm{H}_{2}$ ratio. The best-fit line for the full sample is plotted as a solid black line, while the best-fit line for systems with SFR $>1.0 \mathrm{M}_{\odot} \mathrm{yr}^{-1}$ is given as a dotted line. The best-fit slope and the Spearman rank correlation coefficient for the full set is shown in black (on top), while the values for the high SFR subset are shown in magenta (below). Merger stages 1 and 2 systems are marked as open green triangles. Merger stages 3, 4, and 5 are open cyan diamonds, and merger stages 6 and 7 are identified by blue open squares. AGN are identified by red circles. 

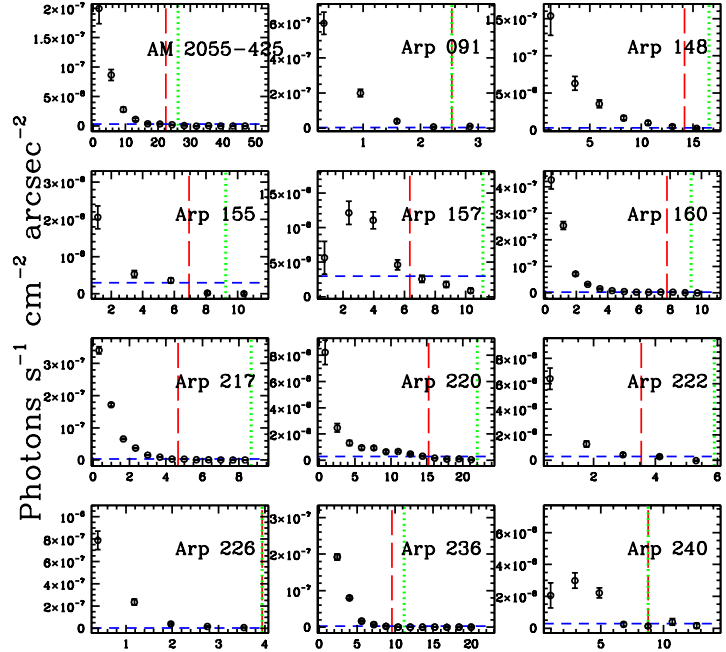

RADIUS ALONG MAJOR AXIS (kpc)

Fig. 5.- Montage of major axis radial profiles from elliptical annuli, plotted against the distance along the major axis. These were obtained using the dmextract software. The blue horizontal line (short dashes) mark the nominal surface brightness cutoff of $3 \times 10^{-9}$ photons s${ }^{-1} \mathrm{~cm}^{-2} \operatorname{arcsec}^{-2}$. The red vertical line (long dashes) mark the 'best' estimate of the radial extent of the X-ray emission, used for the volume determination. The green vertical line (dotted) mark the full $2 \sigma$ extent, for the galaxies with high $\mathrm{S} / \mathrm{N}$ observations. See text for more details.

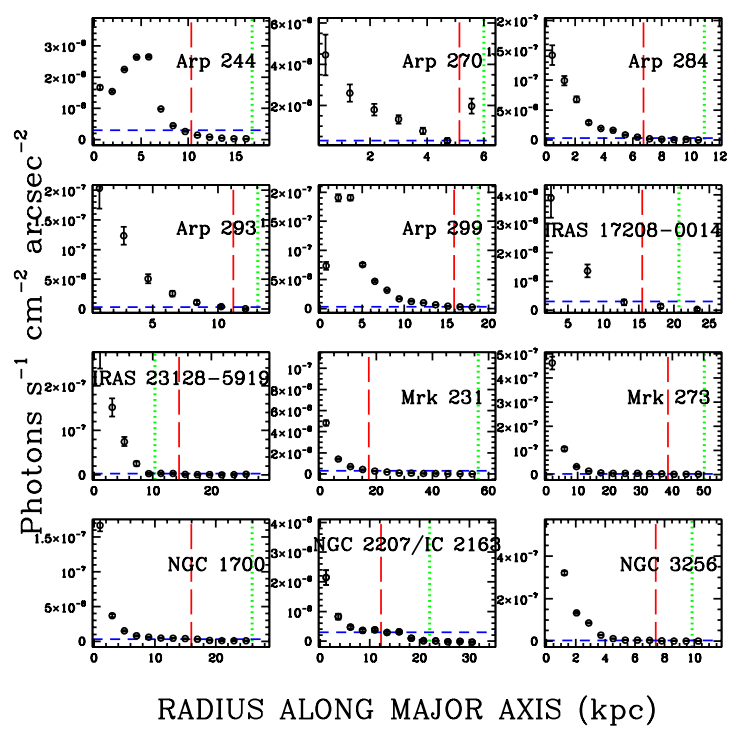

Fig. 6. - Montage of major axis radial profiles from elliptical annuli, plotted against the distance along the major axis. These were obtained using the dmextract software. The blue horizontal line (short dashes) mark the nominal surface brightness cutoff of $3 \times 10^{-9}$ photons s ${ }^{-1} \mathrm{~cm}^{-2} \operatorname{arcsec}^{-2}$. The red vertical line (long dashes) mark the 'best' estimate of the radial extent of the X-ray emission, used for the volume determination. The green vertical line (dotted) mark the full $2 \sigma$ extent, for the galaxies with high $\mathrm{S} / \mathrm{N}$ observations. See text for more details. 


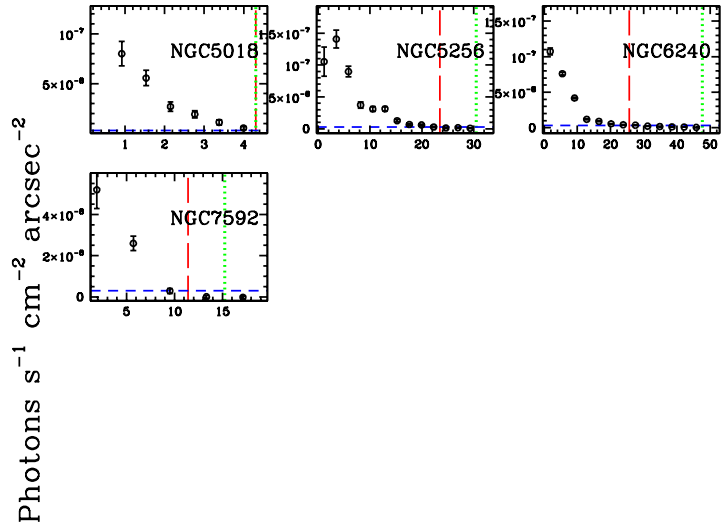

RADIUS ALONG MAJOR AXIS (kpc)

Fig. 7.- Montage of major axis radial profiles from elliptical annuli, plotted against the distance along the major axis. These were obtained using the dmextract software. The blue horizontal line (short dashes) mark the nominal surface brightness cutoff of $3 \times 10^{-9}$ photons s${ }^{-1} \mathrm{~cm}^{-2} \operatorname{arcsec}^{-2}$. The red vertical line (long dashes) mark the 'best' estimate of the radial extent of the X-ray emission, used for the volume determination. The green vertical line (dotted) mark the full $2 \sigma$ extent, for the galaxies with high $\mathrm{S} / \mathrm{N}$ observations. See text for more details.

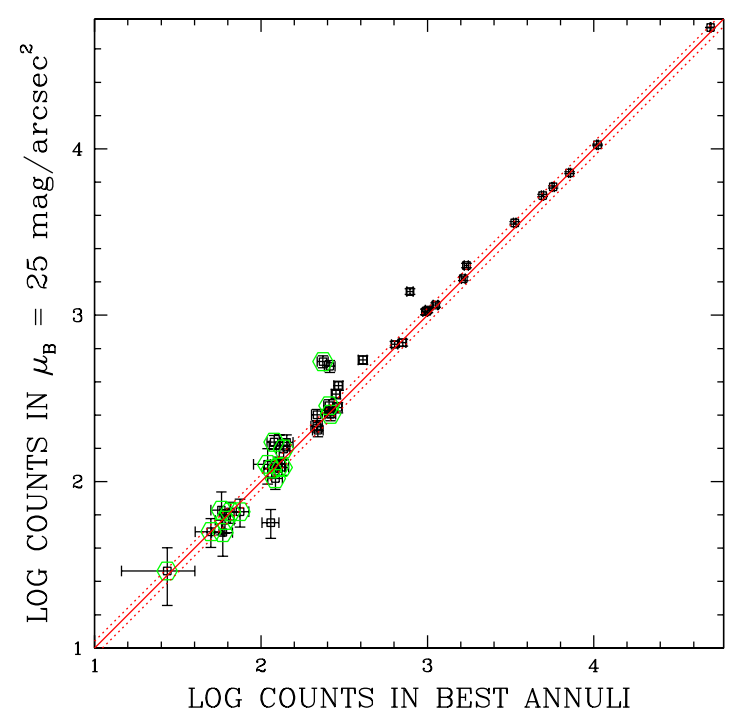

Fig. 8.- Comparison of soft $(0.3-1 \mathrm{keV}) \mathrm{X}$-ray counts within the $\mu_{\mathrm{B}}=25 \mathrm{mag} \operatorname{arcsec}^{-2}$ isophote, and within our best determination of the angular extent of the X-ray emission. The solid line is the one-to-one relation, while the dotted lines represent $\pm 10 \%$ variations. Galaxies marked with open green hexagons are systems with low $\mathrm{S} / \mathrm{N}$ for which we used the single aperture method to get the sizes. 


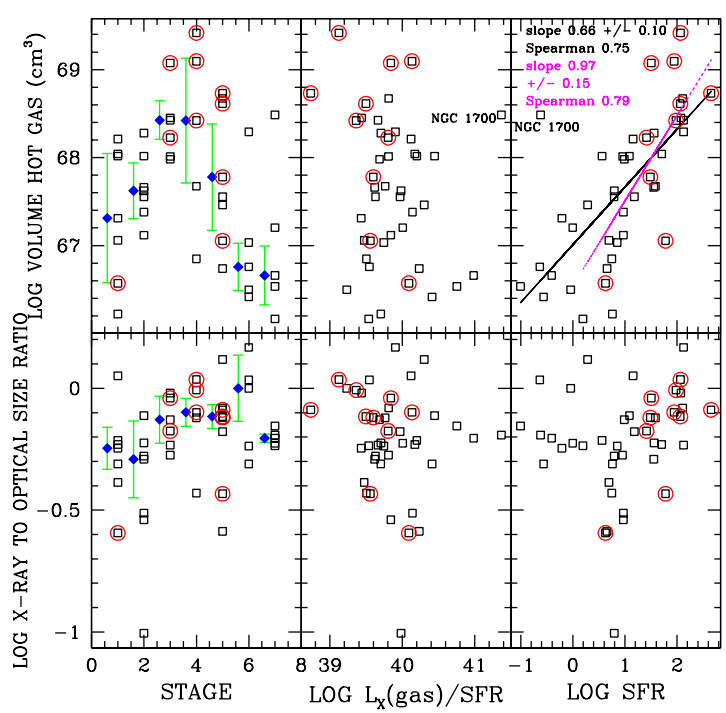

Fig. 9.- Plots of X-ray volume (top row) and $\mathrm{X}$-ray/optical size ratio (bottom row) vs. merger stage (first column), $\mathrm{L}_{\mathrm{X}} / \mathrm{SFR}$ (second column), and SFR (third column). The best-fit line is plotted in the top right panel. Black open squares mark the sample galaxies, with those circled by red circles being AGN. In the left column of plots, the filled blue diamonds are the median values for each stage, slightly offset to the left. The errorbars plotted on the median values are the semiinterquartile range, equal to half the difference between the 75 th percentile and the 25 th percentile.

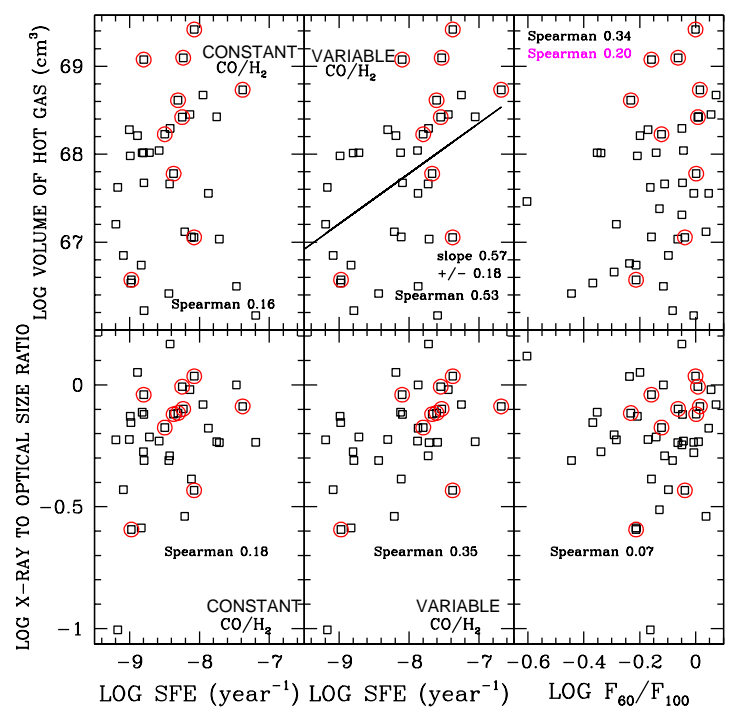

Fig. 10. - Plots of X-ray volume (top row) and $\mathrm{X}$-ray/optical size ratio (bottom row) vs. SFE calculated with constant $\mathrm{CO} / \mathrm{H}_{2}$ ratio (first column), $\mathrm{SFE}$ calculated with variable $\mathrm{CO} / \mathrm{H}_{2}$ ratio (second column), and $\mathrm{F}_{60} / \mathrm{F}_{100}$ ratio (third column). AGN are identified by red circles. 

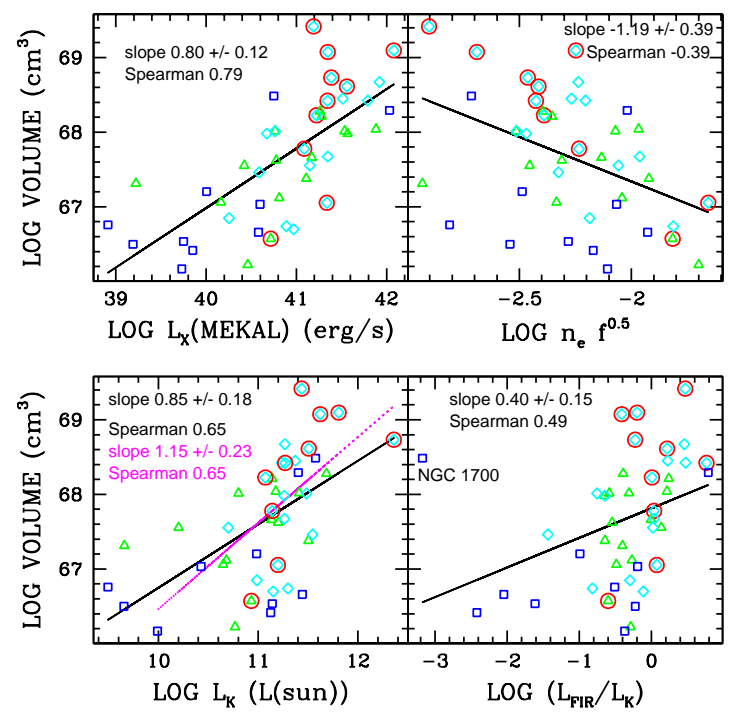

Fig. 11. - Top left: the volume of the hot gas plotted against $\mathrm{L}_{\mathrm{X}}$ (gas). The best-fit line is plotted. Top right: the volume vs. $n_{e} \sqrt{f}$, where $n_{e}$ is the electron density and $\mathrm{f}$ is the filling factor. Bottom left: volume vs. $\mathrm{L}_{\mathrm{K}}$. Bottom right: volume vs. $\mathrm{L}_{\mathrm{FIR}} / \mathrm{L}_{\mathrm{K}}$. Merger stages 1 and 2 systems are marked as open green triangles. Merger stages 3,4 , and 5 are open cyan diamonds, and merger stages 6 and 7 are identified by blue open squares. AGN are identified by red circles.
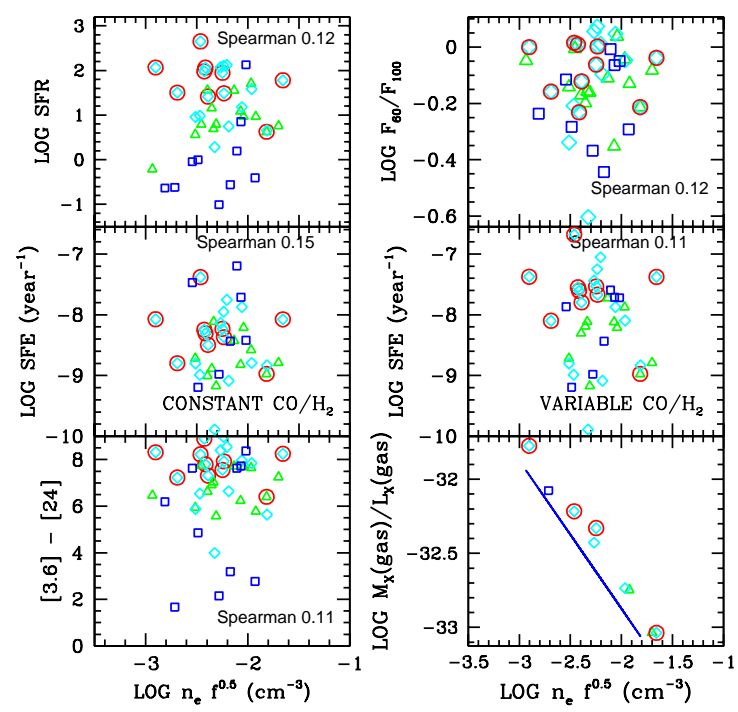

Fig. 12.- Upper left: SFR plotted against $n_{e} \sqrt{f}$. Upper right: $\mathrm{F}_{60} / \mathrm{F}_{100}$ vs. $\mathrm{n}_{\mathrm{e}} \sqrt{f}$. The middle row compares SFE calculated with the two methods with $\mathrm{n}_{\mathrm{e}} \sqrt{f}$. Bottom left: [3.6] $-[24]$ vs. $\mathrm{n}_{\mathrm{e}} \sqrt{f}$. Bottom right: $\mathrm{M}_{\mathrm{X}}$ (gas) $/ \mathrm{L}_{\mathrm{X}}$ (gas) vs. $\mathrm{n}_{\mathrm{e}} \sqrt{f}$ for the 15 systems with measured temperatures. The solid blue line in the lower right plot is the assumed relation assuming a constant temperature of $0.3 \mathrm{keV}$. In these plots, $\mathrm{n}_{\mathrm{e}}$ is the electron density and $\mathrm{f}$ is the filling factor. Merger stages 1 and 2 systems are marked as open green triangles. Merger stages 3, 4, and 5 are open cyan diamonds, and merger stages 6 and 7 are identified by blue open squares. AGN are identified by red circles. 

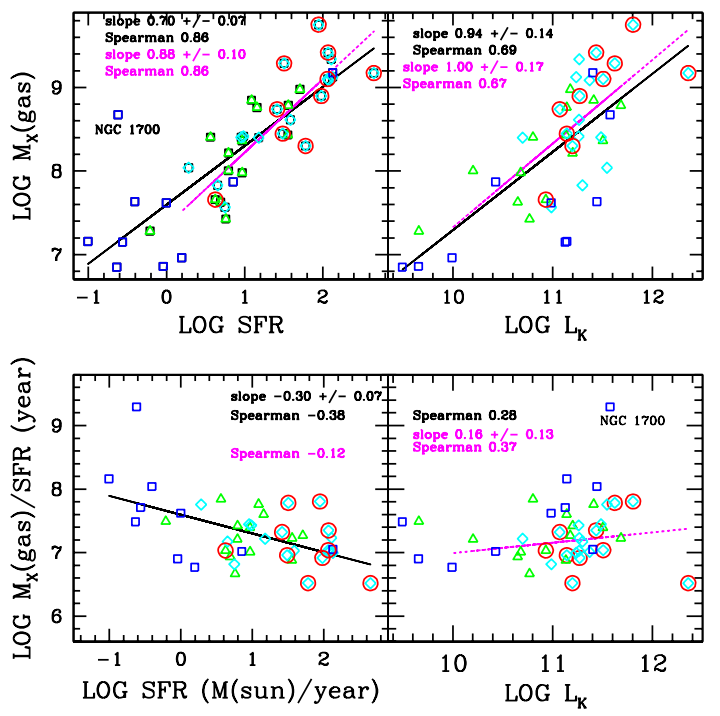

Fig. 13. - $\mathrm{M}_{\mathrm{X}}$ (gas) vs. SFR (top left), $\mathrm{M}_{\mathrm{X}}$ (gas) vs. $\mathrm{L}_{\mathrm{K}}$ (top right), $\mathrm{M}_{\mathrm{X}}$ (gas)/SFR vs. SFR (bottom left), and $\mathrm{M}_{\mathrm{X}}$ (gas)/SFR vs. $\mathrm{L}_{\mathrm{K}}$ (bottom right). Merger stages 1 and 2 systems are marked as open green triangles. Merger stages 3, 4, and 5 are open cyan diamonds, and merger stages 6 and 7 are identified by blue open squares. Spearman rank correlation coefficients for the full dataset are given in black, while the Spearman coefficient for the subset of galaxies with SFR $>1 \mathrm{M}_{\odot} \mathrm{yr}^{-1}$ are given in magenta. When reliable correlations are seen, the best-fit straight line for the full dataset is shown as a solid black line, and for the high SFR subset as a dashed magenta line. AGN are identified by red circles.
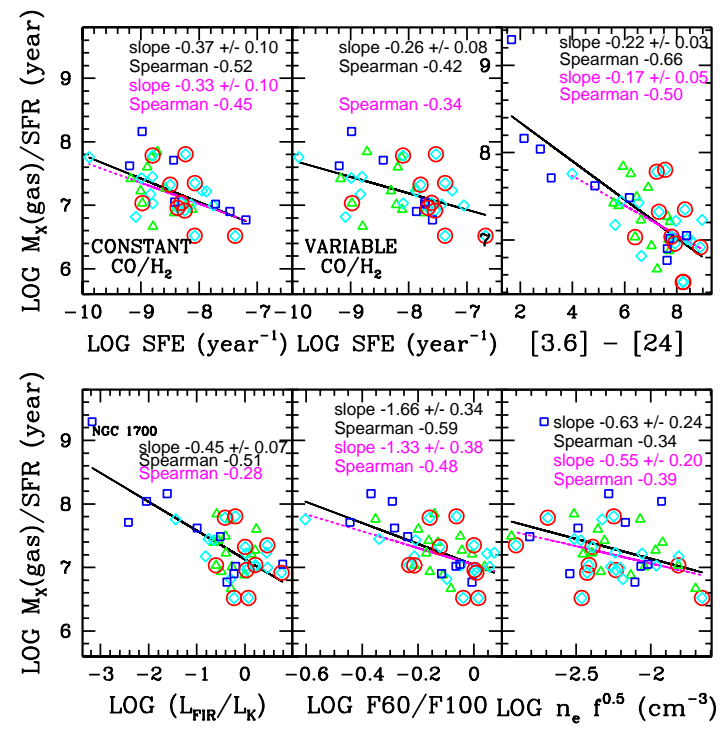

Fig. 14.- $\mathrm{M}_{\mathrm{X}}$ (gas)/SFR vs. the two estimates of SFE (first and second panels, top row), $\mathrm{M}_{\mathrm{X}}$ (gas)/SFR vs. [3.6] $-[24]$ (top right), $\mathrm{M}_{\mathrm{X}}$ (gas)/SFR vs. $\mathrm{L}_{\mathrm{FIR}} / \mathrm{F}_{\mathrm{K}}$ (bottom left), $\mathrm{M}_{\mathrm{X}}$ (gas)/SFR vs. $\mathrm{F}_{60} / \mathrm{F}_{100}$ (bottom middle), and $\mathrm{M}_{\mathrm{X}}$ (gas)/SFR vs. $\mathrm{n}_{\mathrm{e}} \sqrt{f}$ (bottom right). Merger stages 1 and 2 systems are marked as open green triangles. Merger stages 3, 4, and 5 are open cyan diamonds, and merger stages 6 and 7 are identified by blue open squares. Spearman rank correlation coefficients for the full dataset are given in black, while the Spearman coefficient for the subset of galaxies with SFR $>1 \mathrm{M}_{\odot} \mathrm{yr}^{-1}$ are given in magenta. When reliable correlations are seen, the best-fit straight line for the full dataset is shown as a solid black line, and for the high SFR subset as a dashed magenta line. AGN are identified by red circles. 

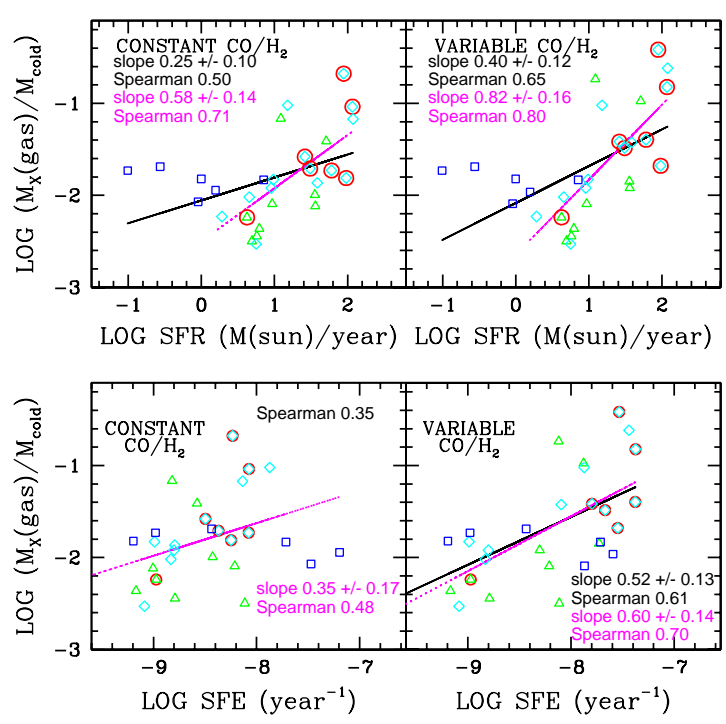

Fig. 15. - Plots of $\mathrm{M}_{\mathrm{X}}$ (gas) $/\left(\mathrm{M}_{\mathrm{HI}}+\mathrm{M}_{\mathrm{H}_{2}}\right)$ vs. SFR (top panels) and SFE (bottom panels). The left column uses a constant $\mathrm{CO} / \mathrm{H}_{2}$ ratio, while the right column uses a variable ratio. Merger stages 1 and 2 systems are marked as open green triangles. Merger stages 3, 4, and 5 are open cyan diamonds, and merger stages 6 and 7 are identified by blue open squares. AGN are identified by red circles. Spearman rank correlation coefficients for the full dataset are given in black, while the Spearman coefficient for the subset of galaxies with SFR > $1 \mathrm{M}_{\odot} \mathrm{yr}^{-1}$ are given in magenta. When reliable correlations are seen, the best-fit straight line for the full dataset is shown as a solid black line, and for the high SFR subset as a dashed magenta line.
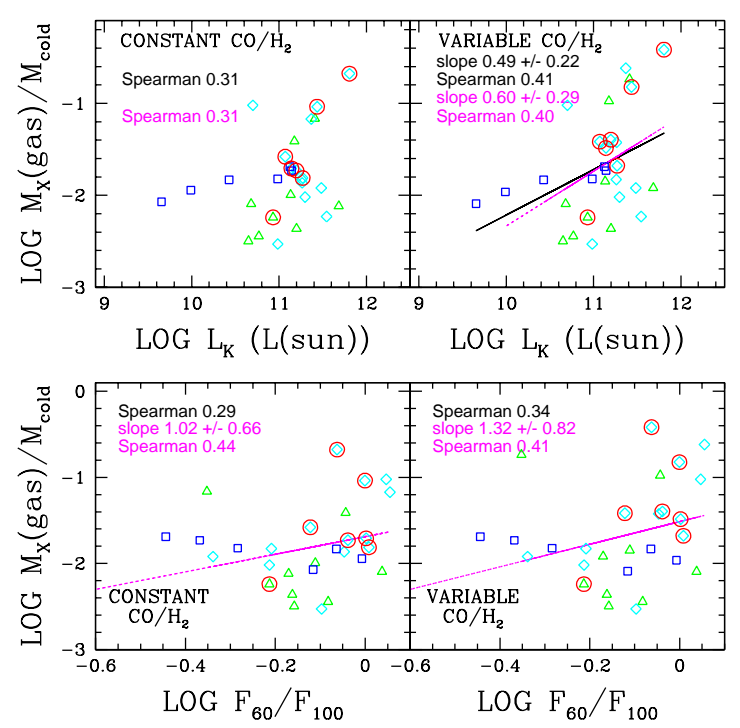

Fig. 16. - Plots of $\mathrm{M}_{\mathrm{X}}$ (gas) $/\left(\mathrm{M}_{\mathrm{HI}}+\mathrm{M}_{\mathrm{H}_{2}}\right)$ vs $\mathrm{L}_{\mathrm{K}}$ (top row) and $\mathrm{F}_{60} / \mathrm{F}_{100}$ (bottom row). The left column uses a constant $\mathrm{CO} / \mathrm{H}_{2}$ ratio, while the right column uses a variable ratio. Merger stages 1 and 2 systems are marked as open green triangles. Merger stages 3, 4, and 5 are open cyan diamonds, and merger stages 6 and 7 are identified by blue open squares. AGN are identified by red circles. Spearman rank correlation coefficients for the full dataset are given in black, while the Spearman coefficient for the subset of galaxies with SFR > $1 \mathrm{M}_{\odot} \mathrm{yr}^{-1}$ are given in magenta. When reliable correlations are seen, the best-fit straight line for the full dataset is shown as a solid black line, and for the high SFR subset as a dashed magenta line. 

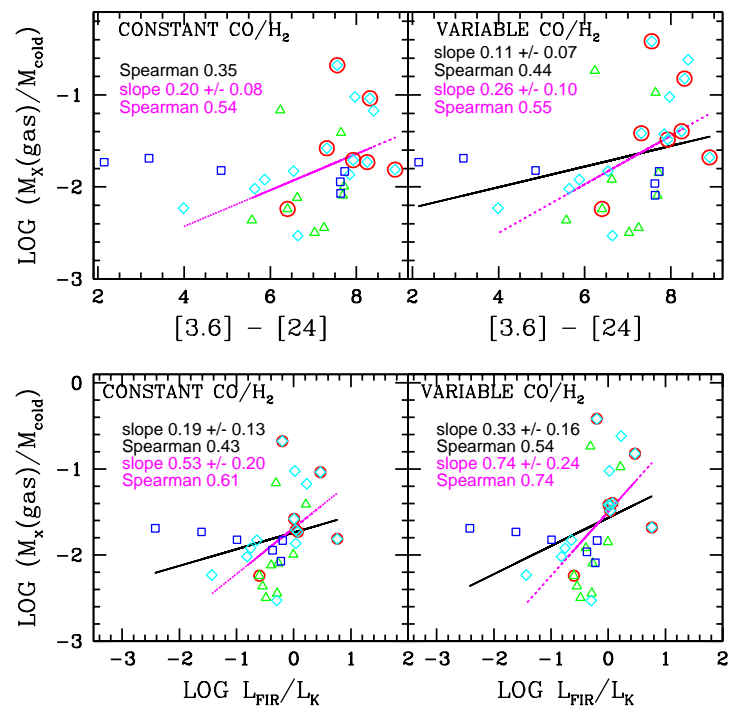

Fig. 17.- Comparisons between $\mathrm{M}_{\mathrm{X}}$ (gas) $/\left(\mathrm{M}_{\mathrm{HI}}+\mathrm{M}_{\mathrm{H}_{2}}\right)$ and [3.6] - [24] (top panels), and $\mathrm{L}_{\mathrm{FIR}} / \mathrm{L}_{\mathrm{K}}$ (bottom panels). The best-fit line for the full sample is plotted as a solid black line, while the best-fit line for systems with SFR $>1.0 \mathrm{M}_{\odot} \mathrm{yr}^{-1}$ is given as a dotted line. The best-fit slope and the Spearman rank correlation coefficient for the full set is shown in black (on top), while the values for the high SFR subset are shown in magenta (below). Merger stages 1 and 2 systems are marked as open green triangles. Merger stages 3,4 , and 5 are open cyan diamonds, and merger stages 6 and 7 are identified by blue open squares. AGN are identified by red circles. NGC 1700 is not plotted. When reliable correlations are seen, the best-fit straight line for the full dataset is shown as a solid black line, and for the high SFR subset as a dashed magenta line. AGN are identified by red circles.
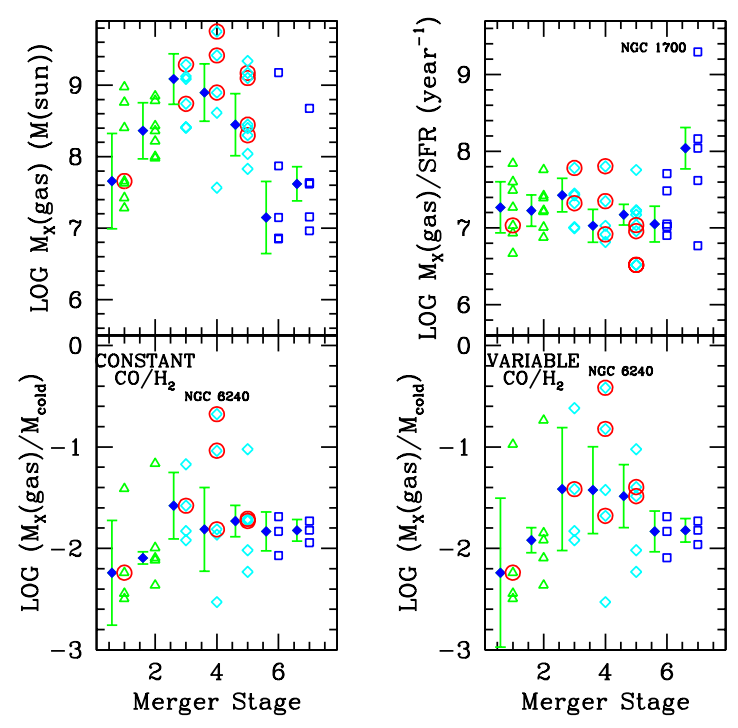

Fig. 18. - Top left: hot gas mass $\mathrm{M}_{\mathrm{X}}$ (gas) vs. merger stage. Top right: $\mathrm{M}_{\mathrm{X}}$ (gas)/SFR vs. merger stage. Bottom row: $\mathrm{M}_{\mathrm{X}}$ (gas) $/ \mathrm{M}_{\text {cold }}=$ $\mathrm{M}_{\mathrm{X}}$ (gas) $/\left(\mathrm{M}_{\mathrm{H}_{2}}+\mathrm{M}_{\mathrm{HI}}\right)$, calculated with a constant $\mathrm{CO} / \mathrm{H}_{2}$ ratio (left panel) and the variable $\mathrm{CO} / \mathrm{H}_{2}$ ratio (right panel). Merger stages 1 and 2 systems are marked as open green triangles. Merger stages 3,4 , and 5 are open cyan diamonds, and merger stages 6 and 7 are identified by blue open squares. AGN are identified by red circles. NGC 1700 is not plotted in the bottom panels due to the lack of a complete set of CO data. The filled blue diamonds are the median values for each stage, slightly offset to the left. The errorbars plotted on the median values are the semi-interquartile range, equal to half the difference between the 75th percentile and the 25 th percentile. 

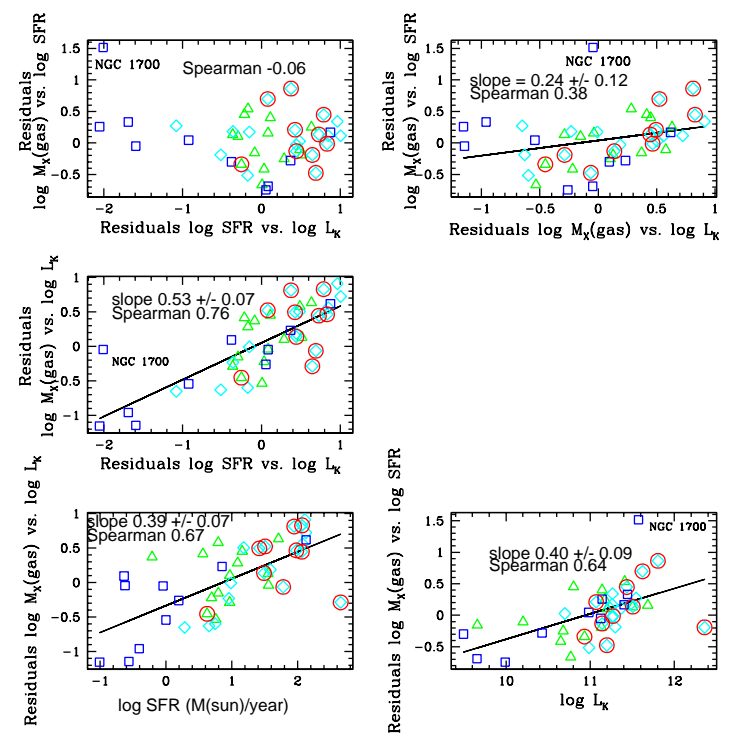

Fig. 19. - A search for correlations between residuals of best-fit plots for various quantities. Residuals are defined as observed - (best-fit value), i.e., a negative residual in the $\log \mathrm{SFR}$ vs. $\log \mathrm{L}_{\mathrm{K}}$ plot means that it has a low $\mathrm{SFR}$ relative to $\mathrm{L}_{\mathrm{K}}$ (that is, a low sSFR or 'post-starburst'). Merger stages 1 and 2 systems are marked as open green triangles. Merger stages 3, 4, and 5 are open cyan diamonds, and merger stages 6 and 7 are identified by blue open squares. AGN are identified by red circles.

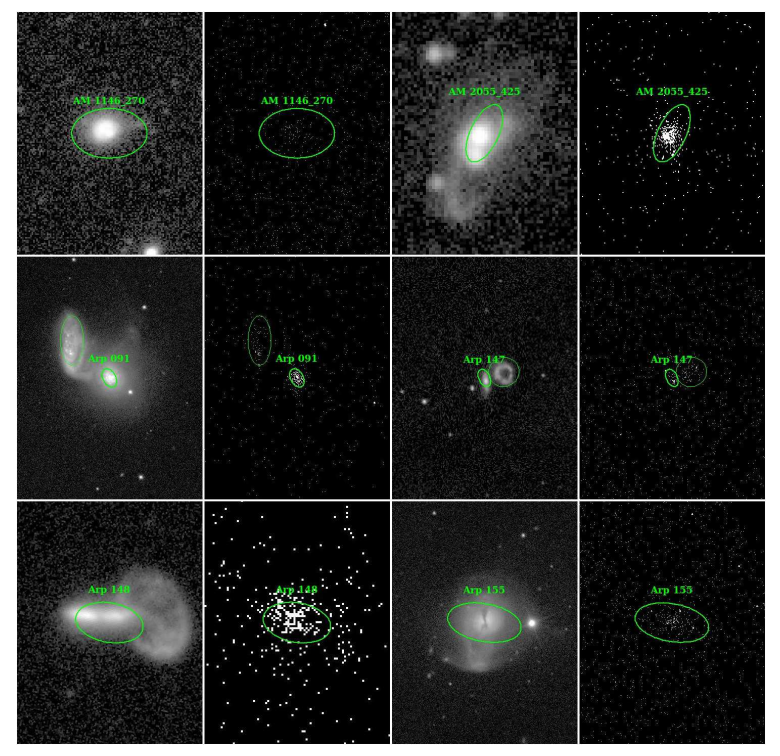

Fig. 20. - Montage of images of the galaxies. The left panel is either the SDSS $\mathrm{g}$ image or the GALEX NUV image (if no SDSS images exist). The right panel is the unsmoothed exposurecorrected Chandra $0.3-1.0 \mathrm{keV}$ low energy map. Logarithmic contours are overlaid in white on the Chandra image, with units of $(3.0,7.6,33.5,178$, and 1000) $\times 10^{-9}$ photons $\mathrm{s}^{-1} \mathrm{~cm}^{-2} \operatorname{arcsec}^{-2}$. The final best-fit ellipse(s) are overlaid in green on both plots. 


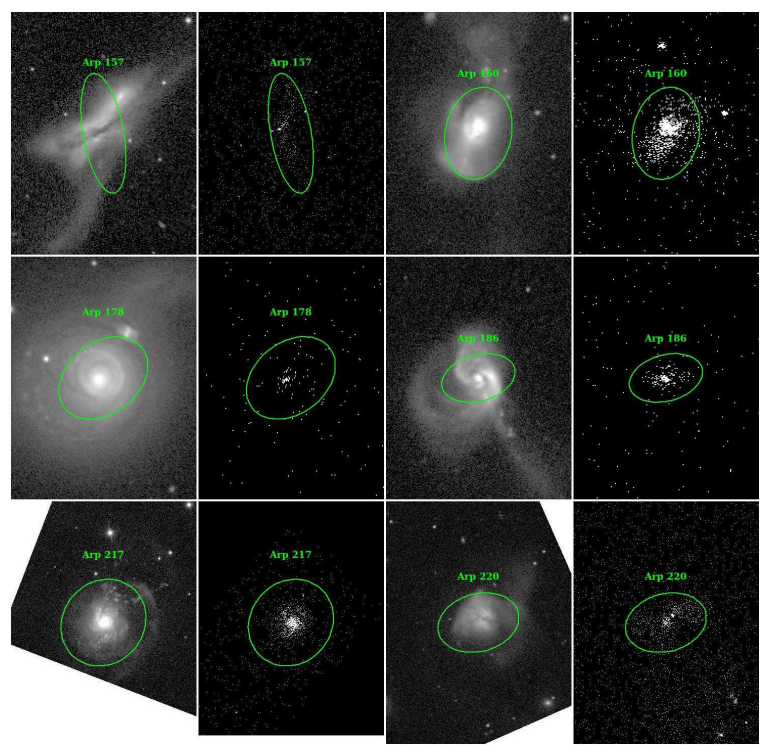

Fig. 21.- Montage of images of the galaxies. The left panel is either the SDSS $g$ image or the GALEX NUV image (if no SDSS images exist). The right panel is the unsmoothed exposurecorrected Chandra $0.3-1.0 \mathrm{keV}$ low energy map. Logarithmic contours are overlaid in white on the Chandra image, with units of $(3.0,7.6,33.5,178$, and 1000) $\times 10^{-9}$ photons $\mathrm{s}^{-1} \mathrm{~cm}^{-2} \operatorname{arcsec}^{-2}$. The final best-fit ellipse(s) are overlaid in green on both plots.

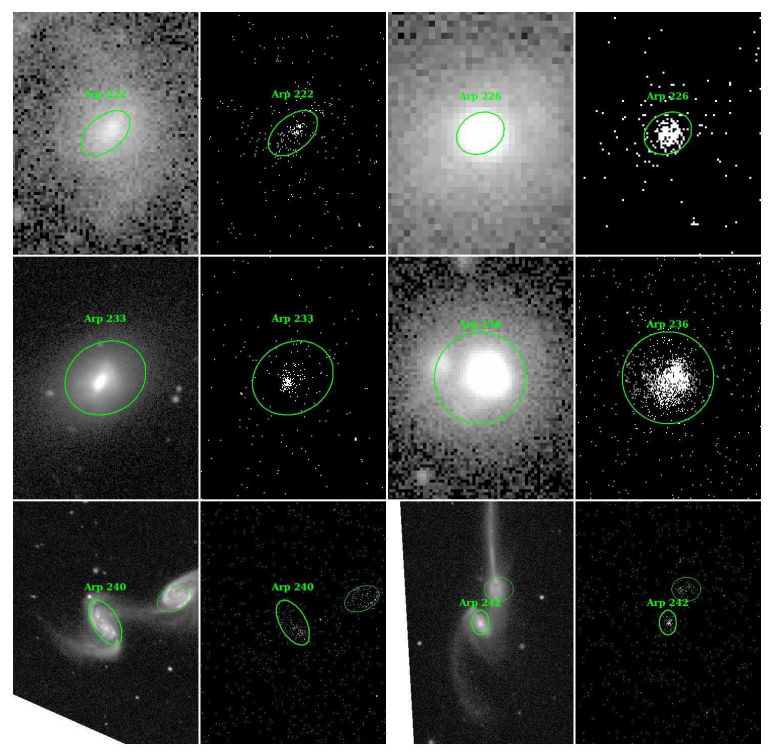

Fig. 22. - Montage of images of the galaxies. The left panel is either the SDSS $\mathrm{g}$ image or the GALEX NUV image (if no SDSS images exist). The right panel is the unsmoothed exposurecorrected Chandra $0.3-1.0 \mathrm{keV}$ low energy map. Logarithmic contours are overlaid in white on the Chandra image, with units of $(3.0,7.6,33.5,178$, and 1000) $\times 10^{-9}$ photons $\mathrm{s}^{-1} \mathrm{~cm}^{-2} \operatorname{arcsec}^{-2}$. The final best-fit ellipse(s) are overlaid in green on both plots. 


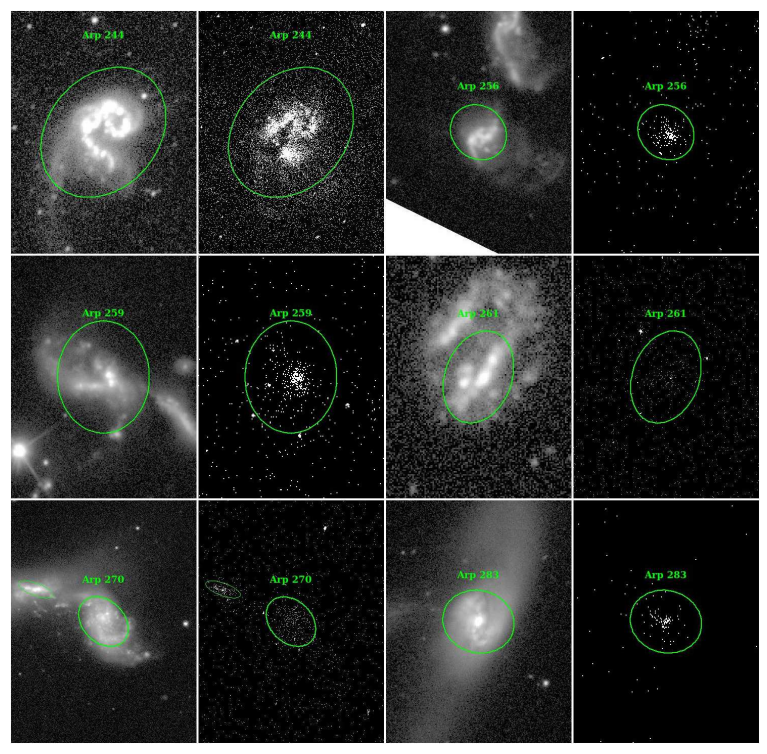

Fig. 23. - Montage of images of the galaxies. The left panel is either the SDSS $g$ image or the GALEX NUV image (if no SDSS images exist). The right panel is the unsmoothed exposurecorrected Chandra $0.3-1.0 \mathrm{keV}$ low energy map. Logarithmic contours are overlaid in white on the Chandra image, with units of $(3.0,7.6,33.5,178$, and 1000) $\times 10^{-9}$ photons $\mathrm{s}^{-1} \mathrm{~cm}^{-2} \operatorname{arcsec}^{-2}$. The final best-fit ellipse(s) are overlaid in green on both plots.

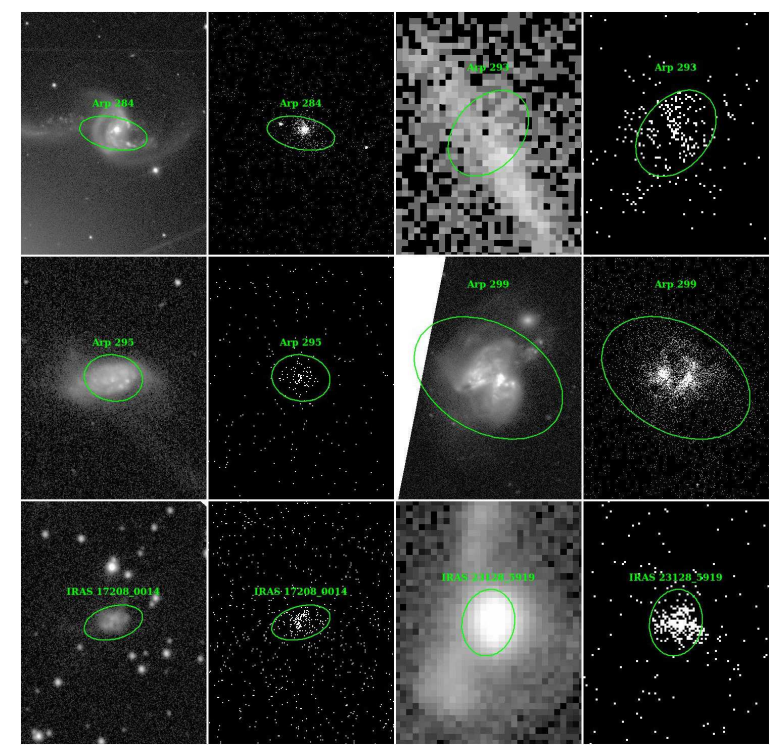

Fig. 24. - Montage of images of the galaxies. The left panel is either the SDSS $\mathrm{g}$ image or the GALEX NUV image (if no SDSS images exist). The right panel is the unsmoothed exposurecorrected Chandra $0.3-1.0 \mathrm{keV}$ low energy map. Logarithmic contours are overlaid in white on the Chandra image, with units of $(3.0,7.6,33.5,178$, and 1000) $\times 10^{-9}$ photons $\mathrm{s}^{-1} \mathrm{~cm}^{-2} \operatorname{arcsec}^{-2}$. The final best-fit ellipse(s) are overlaid in green on both plots. 


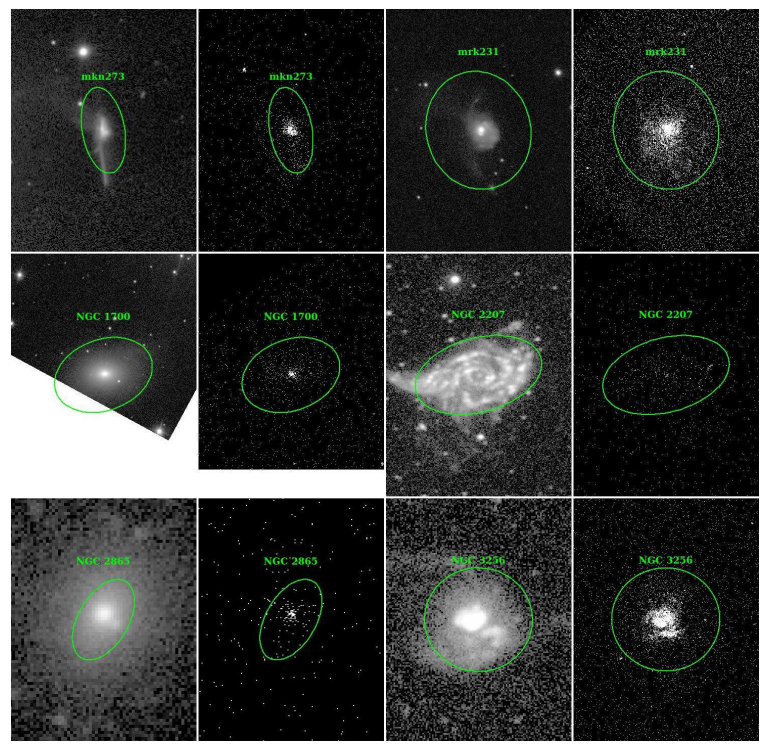

Fig. 25. - Montage of images of the galaxies. The left panel is either the SDSS g image or the GALEX NUV image (if no SDSS images exist). The right panel is the unsmoothed exposurecorrected Chandra $0.3-1.0 \mathrm{keV}$ low energy map. Logarithmic contours are overlaid in white on the Chandra image, with units of $(3.0,7.6,33.5,178$, and 1000) $\times 10^{-9}$ photons $\mathrm{s}^{-1} \mathrm{~cm}^{-2} \operatorname{arcsec}^{-2}$. The final best-fit ellipse(s) are overlaid in green on both plots. 


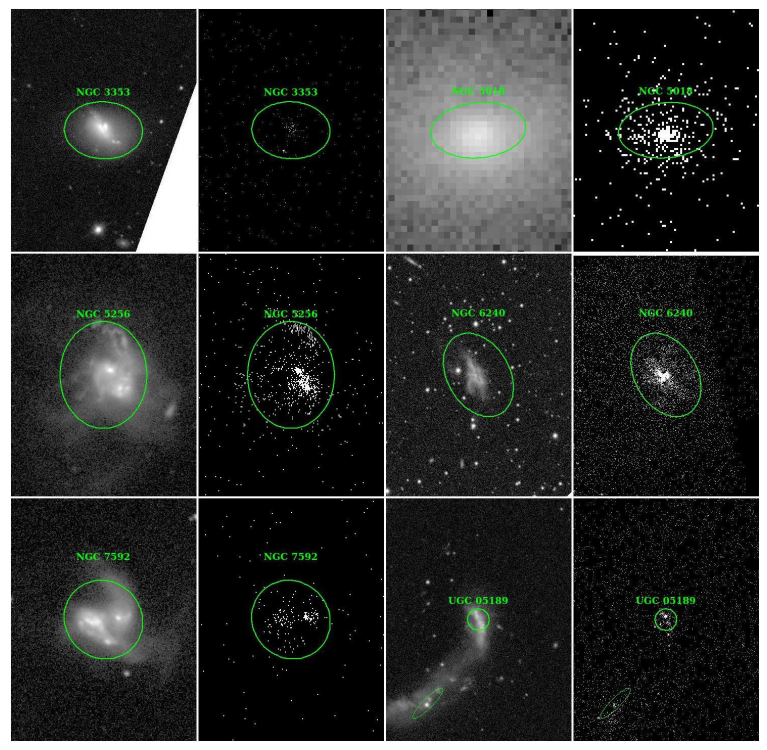

Fig. 26. - Montage of images of the galaxies. The left panel is either the SDSS g image or the GALEX NUV image (if no SDSS images exist). The right panel is the unsmoothed exposurecorrected Chandra $0.3-1.0 \mathrm{keV}$ low energy map. Logarithmic contours are overlaid in white on the Chandra image, with units of $(3.0,7.6,33.5,178$, and 1000) $\times 10^{-9}$ photons $\mathrm{s}^{-1} \mathrm{~cm}^{-2} \operatorname{arcsec}^{-2}$. The final best-fit ellipse(s) are overlaid in green on both plots. 

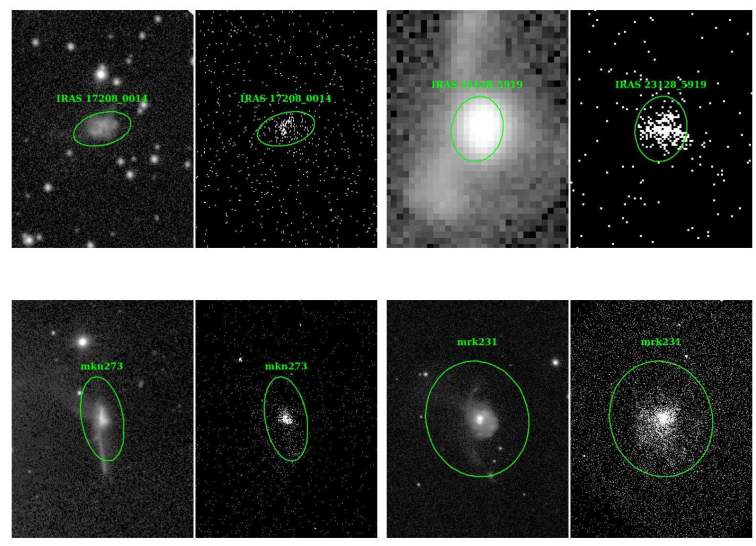

Fig. 27. - Montage of images of the galaxies. The left panel is either the SDSS $g$ image or the GALEX NUV image (if no SDSS images exist). The right panel is the unsmoothed exposurecorrected Chandra $0.3-1.0 \mathrm{keV}$ low energy map. Logarithmic contours are overlaid in white on the Chandra image, with units of $(3.0,7.6,33.5,178$, and 1000) $\times 10^{-9}$ photons $\mathrm{s}^{-1} \mathrm{~cm}^{-2} \operatorname{arcsec}^{-2}$. The final best-fit ellipse(s) are overlaid in green on both plots. 
TABLE 1

Basic Data on Sample Galaxies

\begin{tabular}{|c|c|c|c|c|c|c|c|}
\hline Name & Stage & $\begin{array}{l}\text { Distance } \\
(\mathrm{Mpc})\end{array}$ & $\begin{array}{c}\log \mathrm{L}_{\mathrm{FIR}} \\
\left(\mathrm{L}_{\odot}\right)\end{array}$ & $\begin{array}{c}\log \mathrm{L}_{\mathrm{K}} \\
\left(\mathrm{L}_{\odot}\right)\end{array}$ & $\begin{array}{c}\mathrm{SFR} \\
\left(\mathrm{M}_{\odot} \mathrm{yr}^{-1}\right)\end{array}$ & $\begin{array}{c}\log L_{X}(\text { gas }) \\
\left(\operatorname{erg~s}^{-1}\right)\end{array}$ & AGN? \\
\hline AM 1146-270 & 6 & 24.6 & 8.98 & 9.49 & 0.23 & 38.91 & \\
\hline AM 2055-425 & 5 & 179.1 & 11.72 & 11.27 & 128.23 & 41.92 & \\
\hline Arp 091 & 1 & 34 & 10.33 & 10.93 & 4.22 & 40.72 & Sy2 \\
\hline Arp 147 & 1 & 129 & 10.22 & 10.80 & 3.65 & 40.76 & \\
\hline Arp 148 & 1 & 146.9 & 11.38 & 11.14 & 14.40 & 41.28 & \\
\hline Arp 155 & 7 & 46 & 9.99 & 10.98 & 1.00 & 40.01 & \\
\hline Arp 157 & 4 & 30.5 & 10.69 & 10.99 & 5.61 & 40.25 & \\
\hline Arp 160 & 5 & 39 & 10.72 & 10.70 & 15.14 & 41.15 & \\
\hline Arp 163 & 7 & 23.1 & 9.39 & 9.93 & 1.19 & $<39.57$ & \\
\hline Arp 178 & 5 & 62.1 & 10.11 & 11.54 & 1.92 & 40.59 & \\
\hline Arp 186 & 5 & 64.2 & 11.27 & 11.20 & 60.33 & 41.34 & Sy2:HII \\
\hline Arp 217 & 6 & 18.0 & 10.24 & 10.43 & 7.13 & 40.6 & \\
\hline Arp 220 & 4 & 83 & 12.03 & 11.27 & 95.65 & 41.34 & Sy \\
\hline Arp 222 & 6 & 26.1 & 8.71 & 11.12 & 0.27 & 39.85 & \\
\hline Arp 226 & 5 & 67 & 10.48 & 11.30 & 4.52 & 40.89 & \\
\hline Arp 233 & 7 & 25 & 9.62 & 9.99 & 1.57 & 39.73 & \\
\hline Arp 235 & 7 & 13 & 8.75 & 9.42 & 0.03 & $<39.3$ & \\
\hline Arp 236 & 1 & 81 & 11.39 & 11.18 & 51.02 & 41.88 & \\
\hline Arp 240 & 2 & 102 & 11.29 & 11.68 & 36.18 & 41.26 & \\
\hline Arp 242 & 2 & 98 & 10.65 & 11.20 & 6.23 & 40.78 & \\
\hline Arp 243 & 5 & 79.4 & 11.34 & 11.00 & 21.68 & $<40.41$ & \\
\hline Arp 244 & 3 & 24.1 & 10.62 & 11.26 & 9.70 & 40.67 & \\
\hline Arp 256 & 2 & 109.6 & 11.13 & 11.13 & 35.75 & 41.17 & \\
\hline Arp 259 & 2 & 55 & 10.34 & 10.20 & 6.20 & 40.42 & \\
\hline Arp 261 & 1 & 29 & 9.26 & 9.66 & 0.62 & 39.22 & \\
\hline Arp 263 & 5 & 9.8 & 8.76 & 9.06 & 0.22 & $<38.76$ & \\
\hline Arp 270 & 1 & 29 & 10.16 & 10.65 & 4.93 & 40.17 & \\
\hline Arp 283 & 1 & 30 & 10.48 & 10.77 & 5.74 & 40.46 & \\
\hline Arp 284 & 2 & 39 & 10.41 & 10.68 & 9.31 & 40.81 & \\
\hline Arp 293 & 2 & 82 & 11.10 & 11.41 & 12.24 & 41.54 & Liner/HII \\
\hline Arp 295 & 2 & 94 & 10.86 & 11.50 & 9.29 & 41.11 & \\
\hline Arp 299 & 3 & 48 & 11.60 & 11.37 & 119.01 & 41.51 & \\
\hline IRAS 17208-0014 & 6 & 183 & 12.19 & 11.40 & 133.97 & 42.03 & Liner \\
\hline IRAS 23128-5919 & 3 & 184 & 11.71 & 11.24 & 133.83 & 41.79 & \\
\hline Mrk 231 & 5 & 178.1 & 12.13 & 12.36 & 450.05 & 41.39 & Sy1 \\
\hline Mrk 273 & 4 & 160.5 & 11.90 & 11.44 & 116.83 & 41.19 & Sy2 \\
\hline NGC 034 & 5 & 79.3 & 11.18 & 11.14 & 30.78 & 41.09 & Sy2 \\
\hline NGC 1700 & 7 & 52.5 & 8.40 & 11.57 & 0.24 & 40.75 & \\
\hline NGC 2207/IC 2163 & 3 & 38.0 & 10.73 & 11.48 & 9.03 & 40.76 & \\
\hline
\end{tabular}


TABLE 1-Continued

\begin{tabular}{ccrrrrrr}
\hline \hline Name & Stage & $\begin{array}{c}\text { Distance } \\
(\mathrm{Mpc})\end{array}$ & $\begin{array}{c}\log \mathrm{L}_{\mathrm{FIR}} \\
\left(\mathrm{L}_{\odot}\right)\end{array}$ & $\begin{array}{c}\log \mathrm{L}_{\mathrm{K}} \\
\left(\mathrm{L}_{\odot}\right)\end{array}$ & $\begin{array}{c}\mathrm{SFR} \\
\left(\mathrm{M}_{\odot} \mathrm{yr}^{-1}\right)\end{array}$ & $\begin{array}{c}\log \mathrm{L}_{\mathrm{X}}(\mathrm{gas}) \\
\left(\mathrm{erg} \mathrm{s}^{-1}\right)\end{array}$ & AGN? \\
\hline NGC 2865 & 7 & 37.9 & 9.53 & 11.14 & 0.10 & 39.75 & \\
NGC 3256 & 4 & 37.0 & 11.30 & 11.26 & 38.50 & 41.35 & \\
NGC 3353 & 6 & 18.5 & 9.43 & 9.66 & 0.91 & 39.19 & \\
NGC 5018 & 7 & 38.4 & 9.40 & 11.44 & 0.39 & 40.58 & \\
NGC 5256 & 3 & 120.9 & 11.21 & 11.62 & 32.02 & 41.35 & Sy2 \\
NGC 6240 & 4 & 108.8 & 11.61 & 11.81 & 88.46 & 42.08 & Sy2/Liner \\
NGC 7592 & 3 & 99.5 & 11.08 & 11.07 & 26.08 & 41.22 & Sy2 \\
UGC 2238 & 5 & 87.1 & 11.04 & 11.15 & 9.41 & 40.97 & Liner \\
UGC 5101 & 5 & 164.3 & 11.72 & 11.51 & 115.63 & 41.56 & Sy1 \\
UGC 5189 & 2 & 48.9 & 9.48 & 9.47 & 1.54 & $<39.26$ & \\
\hline
\end{tabular}


TABLE 2

Global Molecular and Atomic Gas Mass in the Sample Galaxies

\begin{tabular}{|c|c|c|c|c|c|c|c|c|c|c|}
\hline Name & $\begin{array}{l}\mathrm{LOG} \\
\mathrm{M}_{\mathrm{H}_{2}}{ }^{\dagger} \\
\left(\mathrm{M}_{\odot}\right)\end{array}$ & $\begin{array}{l}\mathrm{LOG} \\
\mathrm{M}_{\mathrm{H}_{2}} \ddagger \\
\left(\mathrm{M}_{\odot}\right)\end{array}$ & $\begin{array}{c}\mathrm{CO} \\
\mathrm{REF}^{*}\end{array}$ & $\begin{array}{c}\mathrm{LOG} \\
\mathrm{SFE}^{\dagger} \\
\left(\mathrm{yr}^{-1}\right)\end{array}$ & $\begin{array}{l}\mathrm{LOG} \\
\mathrm{SFE}^{\ddagger} \\
\left(\mathrm{yr}^{-1}\right)\end{array}$ & $\begin{array}{c}\mathrm{LOG} \\
\mathrm{M}_{\mathrm{HI}} \\
\left(\mathrm{M}_{\odot}\right)\end{array}$ & $\begin{array}{c}\mathrm{HI} \\
\mathrm{REF}^{* *}\end{array}$ & $\begin{array}{l}\mathrm{LOG} \\
\mathrm{M}_{\text {hot }} \\
\left(\mathrm{M}_{\odot}\right)\end{array}$ & $\begin{array}{c}\mathrm{LOG} \\
\mathrm{M}_{\text {hot }} / \mathrm{M}_{\text {cold }}^{\dagger}\end{array}$ & $\begin{array}{c}\mathrm{LOG} \\
\log \mathrm{M}_{\mathrm{hot}} / \mathrm{M}_{\mathrm{cold}} \ddagger\end{array}$ \\
\hline AM $1146-270$ & & & & & & 9.15 & 16 & 6.85 & & \\
\hline AM 2055-425 & 10.06 & 9.36 & 1 & -7.94 & -7.24 & & & 9.34 & & \\
\hline Arp 091 & 9.60 & 9.60 & 2 & -8.96 & -8.96 & 9.60 & 17 & 7.66 & -2.23 & -2.23 \\
\hline Arp 147 & 9.28 & 9.28 & 3 & -8.71 & -8.71 & & & 8.40 & & \\
\hline Arp 148 & 10.05 & 9.35 & 4 & -8.88 & -8.18 & & & 8.76 & & \\
\hline Arp 155 & 9.19 & 9.19 & 5 & -9.18 & -9.18 & 9.08 & 17 & 7.62 & -1.81 & -1.81 \\
\hline Arp 157 & 9.84 & 9.84 & 6 & -9.08 & -9.08 & 9.75 & 17 & 7.57 & -2.52 & -2.52 \\
\hline Arp 160 & 9.05 & 9.05 & 6 & -7.86 & -7.86 & 9.18 & 17 & 8.40 & -1.01 & -1.01 \\
\hline Arp 163 & & & & & & 9.08 & 17 & & & \\
\hline Arp 178 & 10.17 & 10.17 & 2 & -9.88 & -9.88 & 9.58 & 17 & 8.04 & -2.22 & -2.22 \\
\hline Arp 186 & 9.86 & 9.16 & 4 & -8.07 & -7.37 & 9.55 & 18 & 8.30 & -1.72 & -1.39 \\
\hline Arp 217 & 8.57 & 8.57 & 7 & -7.7 & -7.7 & 9.67 & 17 & 7.87 & -1.82 & -1.82 \\
\hline Arp 220 & 10.23 & 9.53 & 4 & -8.24 & -7.54 & 10.54 & 17 & 8.90 & -1.80 & -1.67 \\
\hline Arp 222 & 7.87 & 7.87 & 8 & -8.43 & -8.43 & 8.79 & 17 & 7.15 & -1.68 & -1.68 \\
\hline Arp 226 & 9.49 & 9.49 & 8 & -8.82 & -8.82 & 9.60 & 18 & 7.83 & -2.01 & -2.01 \\
\hline Arp 233 & 7.39 & 7.79 & 9 & -7.19 & -7.58 & 8.89 & 17 & 6.96 & -1.93 & -1.95 \\
\hline Arp 235 & & & & & & 8.85 & 17 & & & \\
\hline Arp 236 & 10.28 & 9.59 & 4 & -8.57 & -7.87 & 9.71 & 19 & 8.98 & -1.40 & -0.97 \\
\hline Arp 240 & 10.56 & 9.86 & 10 & -8.99 & -8.29 & 10.64 & 17 & 8.79 & -2.11 & -1.91 \\
\hline Arp 242 & 9.96 & 9.96 & 10 & -9.16 & -9.16 & 10.46 & 17 & 8.22 & -2.35 & -2.35 \\
\hline Arp 243 & 9.63 & 8.93 & 6 & -8.28 & -7.59 & 9.50 & 20 & & & \\
\hline Arp 244 & 9.98 & 9.98 & 10 & -8.98 & -8.98 & 9.90 & 21 & 8.41 & -1.82 & -1.82 \\
\hline Arp 256 & 9.98 & 9.28 & 10 & -8.42 & -7.72 & 10.23 & 17 & 8.43 & -1.98 & -1.84 \\
\hline Arp 259 & & & & & & 10.21 & 17 & 8.00 & & \\
\hline Arp 261 & & & & & & 9.75 & 17 & 7.28 & & \\
\hline Arp 263 & & & & & & 9.25 & 17 & & & \\
\hline Arp 270 & 8.81 & 8.81 & 2 & -8.1 & -8.1 & 10.10 & 17 & 7.63 & -2.49 & -2.49 \\
\hline Arp 283 & 9.55 & 9.55 & 7 & -8.78 & -8.78 & 9.58 & 17 & 7.43 & -2.43 & -2.43 \\
\hline
\end{tabular}


TABLE 2-Continued

\begin{tabular}{|c|c|c|c|c|c|c|c|c|c|c|}
\hline Name & $\begin{array}{l}\mathrm{LOG} \\
\mathrm{M}_{\mathrm{H}_{2}}^{\dagger} \\
\left(\mathrm{M}_{\odot}\right)\end{array}$ & $\begin{array}{l}\mathrm{LOG} \\
\mathrm{M}_{\mathrm{H}_{2}} \ddagger \\
\left(\mathrm{M}_{\odot}\right)\end{array}$ & $\begin{array}{c}\mathrm{CO} \\
\mathrm{REF}^{*}\end{array}$ & $\begin{array}{l}\mathrm{LOG} \\
\mathrm{SFE}^{\dagger} \\
\left(\mathrm{yr}^{-1}\right)\end{array}$ & $\begin{array}{c}\mathrm{LOG} \\
\mathrm{SFE}^{\ddagger} \\
\left(\mathrm{yr}^{-1}\right)\end{array}$ & $\begin{array}{c}\mathrm{LOG} \\
\mathrm{M}_{\mathrm{HI}} \\
\left(\mathrm{M}_{\odot}\right)\end{array}$ & $\begin{array}{c}\mathrm{HI} \\
\mathrm{REF}^{* *}\end{array}$ & $\begin{array}{l}\mathrm{LOG} \\
\mathrm{M}_{\text {hot }} \\
\left(\mathrm{M}_{\odot}\right)\end{array}$ & $\begin{array}{c}\mathrm{LOG} \\
\mathrm{M}_{\text {hot }} / \mathrm{M}_{\text {cold }}^{\dagger}\end{array}$ & $\begin{array}{c}\mathrm{LOG} \\
\log \mathrm{M}_{\mathrm{hot}} / \mathrm{M}_{\text {cold }}{ }^{\ddagger}\end{array}$ \\
\hline Arp 284 & 9.18 & 9.18 & 6 & -8.2 & -8.2 & 10.01 & 17 & 7.98 & -2.08 & -2.08 \\
\hline Arp 293 & 9.91 & 9.21 & 4 & -8.81 & -8.11 & 9.35 & 22 & 8.85 & -1.15 & -0.73 \\
\hline Arp 295 & & & & & & 10.40 & 23 & 8.36 & & \\
\hline Arp 299 & 10.21 & 9.51 & 10 & -8.13 & -7.43 & 9.25 & 17 & 9.09 & -1.16 & -0.61 \\
\hline IRAS 17208-0014 & 10.55 & 9.85 & 11 & -8.41 & -7.71 & & & 9.18 & & \\
\hline IRAS 23128-5919 & 9.88 & 9.18 & 1 & -7.74 & -7.04 & & & 9.12 & & \\
\hline Mrk 231 & 10.03 & 9.34 & 6 & -7.37 & -6.67 & & & 9.17 & & \\
\hline Mrk 273 & 10.14 & 9.44 & 4 & -8.06 & -7.36 & 10.16 & 17 & 9.42 & -1.03 & -0.81 \\
\hline NGC 034 & 9.86 & 9.16 & 8 & -8.36 & -7.66 & 9.85 & 26 & 8.45 & -1.70 & -1.47 \\
\hline NGC 1700 & & & & & & & & 8.67 & & \\
\hline NGC $2207 /$ IC 2163 & 9.76 & 9.76 & 15 & -8.79 & -8.79 & 10.19 & 17 & 8.40 & -1.91 & -1.91 \\
\hline NGC 2865 & 7.97 & 7.97 & 12 & -8.97 & -8.97 & 8.83 & 24 & 7.16 & -1.72 & -1.72 \\
\hline NGC 3256 & 10.38 & 9.68 & 8 & -8.78 & -8.08 & 9.79 & 25 & 8.61 & -1.85 & -1.41 \\
\hline NGC 3353 & 7.43 & 7.83 & 14 & -7.46 & -7.86 & 8.92 & 17 & 6.86 & -2.06 & -2.08 \\
\hline NGC 5018 & & & & & & 8.82 & 17 & 7.64 & & \\
\hline NGC 5256 & 10.30 & 9.60 & 13 & -8.79 & -8.09 & & & 9.29 & & \\
\hline NGC 6240 & 10.18 & 9.48 & 6 & -8.22 & -7.52 & 10.07 & 17 & 9.75 & -0.67 & -0.41 \\
\hline NGC 7592 & 9.91 & 9.21 & 10 & -8.49 & -7.79 & 10.10 & 17 & 8.74 & -1.57 & -1.41 \\
\hline UGC 2238 & 9.97 & 9.27 & 4 & -8.98 & -8.28 & 9.98 & 17 & & & \\
\hline UGC 5101 & 10.37 & 9.67 & 4 & -8.3 & -7.6 & & & 9.10 & & \\
\hline UGC 5189 & & & & & & 10.14 & 17 & & & \\
\hline
\end{tabular}

${ }^{\dagger}$ Assuming the standard Galactic $\mathrm{CO} / \mathrm{H}_{2}$ ratio. SFE in this paper is defined as $\mathrm{SFR} / \mathrm{M}_{\mathrm{H}_{2}}$.

${ }^{\ddagger} \mathrm{Using}$ the variable $\mathrm{CO} / \mathrm{H}_{2}$ ratio (see text for details). SFE is defined as $\mathrm{SFR} / \mathrm{M}_{\mathrm{H}_{2}}$.

${ }^{*} \mathrm{CO}$ references: 1: Mirabel et al. 1990; 2: Zhu et al. 1999; 3: Horellou et al. 1995; 4: Larson et al. 2016; 5: Wiklind. Combes. \& Henkel 1995; 6: Sanders et al. 1991; 7: Young et al. 1996; 8: Ueda et al. 2014; 9: Israel 2005; 10: 
Bushouse et al. 1999; 11: Solomon et al. 1997; 12: Georgakakis et al. 2001; 13: Papadopoulos et al. 2012; 14: Sage et al. 1992; 15: Elmegreen et al. 2016.

**HI references: 16: Doyle et al. 2005 17: Huchtmeier \& Richter 1989; 18: Obreschkow \& Rawlings 2009; 19: Martin et al. 1991; 20: Bushouse 1987; 21: Gordon. Koribalski. \& Jones 2001 22: van Driel et al. 2000; 23: Hibbard \& van Gorkom 1996; 24: Cox \& Sparke 2004; 25: English et al. 2003; 26: Fernández et al. 2014; 
TABle 3

Final Ellipses Used for Volume Calculations: At $0.3-1.0 \mathrm{keV}$ Surface Brightness of $3 \times$ $10^{-9}$ PHotons S $^{-1} \mathrm{CM}^{-1}$ ARCSEC $^{-2}$

\begin{tabular}{|c|c|c|c|c|c|c|c|c|c|c|}
\hline Name & $\begin{array}{c}\text { Individual } \\
\text { Galaxy }\end{array}$ & $\begin{array}{c}\mathrm{RA} \\
(\mathrm{J} 2000)\end{array}$ & $\begin{array}{c}\text { DEC } \\
(\mathrm{J} 2000)\end{array}$ & $\begin{array}{c}\text { Major } \\
\text { Axis } \\
\text { Radius } \\
\quad\left({ }^{\prime \prime}\right)\end{array}$ & $\begin{array}{c}\text { Minor } \\
\text { Axis } \\
\text { Radius } \\
\quad\left({ }^{\prime \prime}\right)\end{array}$ & $\begin{array}{c}\text { Major } \\
\text { Axis } \\
\text { Radius } \\
\text { (kpc) }\end{array}$ & $\begin{array}{c}\text { Minor } \\
\text { Axis } \\
\text { Radius } \\
\text { (kpc) }\end{array}$ & $\begin{array}{l}\text { P.A. }{ }^{1} \\
(\mathrm{deg})\end{array}$ & $\begin{array}{c}\text { Number } \\
\text { Annuli }\end{array}$ & $\begin{array}{c}\text { Diffuse } \\
0.3- \\
1.0 \mathrm{keV} \\
\text { Counts }\end{array}$ \\
\hline AM 1146-270 & ESO 504-G017 & 114846.046 & -272249.11 & $36 ! 74$ & $24^{\prime \prime} 15$ & 4.38 & 2.88 & 0 & 1 & $27 \pm 13$ \\
\hline AM 2055-425 & ESO 286-IG019 & 205826.554 & -423858.11 & $25^{\prime \prime} 88$ & $11^{\prime \prime} 80$ & 22.48 & 10.25 & 65 & 13 & $289 \pm 19$ \\
\hline Arp 091 & NGC 5953 & 153432.396 & +151136.55 & $15^{\prime \prime} 42$ & $99^{\prime \prime} 90$ & 2.54 & 1.63 & 300 & 5 & $233 \pm 17$ \\
\hline & NGC 5954 & 153434.911 & +151212.96 & $24^{\prime \prime} 17$ & $11^{\prime \prime} 90$ & 3.99 & 1.83 & 90 & 1 & $59 \pm 8$ \\
\hline Arp 147 & IC 298 NED02 & 31119.621 & +1 1851.35 & 8.95 & $5 ! .40$ & 5.60 & 3.38 & 115 & 1 & $60 \pm 7$ \\
\hline & IC 298 & 31118.367 & +11857.12 & $15^{\prime \prime} 00$ & $14^{\prime \prime} 40$ & 9.38 & 8.77 & 20 & 1 & $56 \pm 9$ \\
\hline Arp 148 & NED01 & 110353.839 & +405058.26 & $19^{\prime \prime} 92$ & $11^{\prime \prime} 51$ & 14.19 & 8.20 & 350 & 7 & $216 \pm 17$ \\
\hline Arp 155 & NGC 3656 & 112338.541 & +535030.51 & $31^{\prime \prime} 09$ & $15^{\prime \prime} 81$ & 6.94 & 3.53 & 350 & 5 & $129 \pm 17$ \\
\hline Arp 157 & NGC 520 & 12434.398 & +34727.11 & $42 . ! 92$ & $14^{\prime \prime} .26$ & 6.35 & 2.11 & 100 & 7 & $259 \pm 19$ \\
\hline Arp 160 & NGC 4194 & 121409.490 & +543132.96 & $41^{\prime \prime} 17$ & $29^{\prime \prime} .27$ & 7.79 & 5.54 & 258 & 13 & $1007 \pm 35$ \\
\hline Arp 178 & NGC 5614 & 142407.420 & +345132.48 & $233^{\prime \prime} 87$ & $17^{\prime \prime} 33$ & 7.19 & 5.22 & 38 & 1 & $58 \pm 8$ \\
\hline Arp 186 & NGC 1614 & 43400.068 & -83444.83 & $18^{\prime \prime} 17$ & $11^{\prime \prime} 25$ & 5.66 & 3.50 & 195 & 1 & $122 \pm 12$ \\
\hline Arp 217 & NGC 3310 & 103845.964 & +533008.73 & 53 .' 48 & $48^{\prime \prime} \cdot 04$ & 4.67 & 4.19 & 230 & 13 & $4924 \pm 73$ \\
\hline Arp 220 & IC 4553 & 153457.578 & +233006.07 & $37 ! .87$ & $26^{\prime \prime} 55$ & 15.25 & 10.69 & 15 & 13 & $711 \pm 32$ \\
\hline Arp 222 & NGC 7727 & 233953.876 & -121734.61 & $27 ! 98$ & $16^{\prime \prime} 53$ & 3.54 & 2.09 & 40 & 5 & $143 \pm 13$ \\
\hline Arp 226 & NGC 7252 & 222044.774 & -244041.77 & $12 . ! 13$ & $9{ }^{\prime \prime} 67$ & 3.94 & 3.14 & 30 & 5 & $217 \pm 16$ \\
\hline Arp 233 & UGC 05720 & 103231.589 & +542405.25 & $20 ! 41$ & $17^{\prime \prime} \cdot 20$ & 2.48 & 2.09 & 30 & 1 & $127 \pm 12$ \\
\hline Arp 236 & IC 1623 & 010746.897 & -173026.91 & $24^{\prime \prime} 45$ & $24^{\prime \prime} 37$ & 9.60 & 9.57 & 90 & 13 & $1645 \pm 42$ \\
\hline \multirow[t]{2}{*}{ Arp 240} & NGC 5258 & 133957.690 & +04956.55 & $24^{\prime \prime} 14$ & $12^{\prime \prime} 50$ & 11.94 & 6.18 & 300 & 1 & $130 \pm 13$ \\
\hline & NGC 5257 & 133953.221 & +0 5019.82 & $23^{\prime \prime} 63$ & $15^{\prime \prime} 50$ & 11.69 & 7.55 & 25 & 7 & $127 \pm 14$ \\
\hline \multirow[t]{2}{*}{ Arp 242} & NGC $4676 \mathrm{~B}$ & 124611.236 & +304322.77 & $11^{\prime \prime} 95$ & $8^{\prime \prime} .28$ & 5.68 & 3.93 & 90 & 1 & $69 \pm 9$ \\
\hline & NGC $4676 \mathrm{~A}$ & 124609.850 & +304355.24 & $14^{\prime \prime} 04$ & $11^{\prime \prime} 28$ & 6.67 & 5.59 & 0 & 1 & $68 \pm 9$ \\
\hline Arp 244 & NGC $4038 / 9$ & 120153.451 & -185226.50 & $88^{\prime \prime} \cdot 32$ & $69^{\prime \prime} 12$ & 10.32 & 8.08 & 50 & 13 & $50307 \pm 248$ \\
\hline Arp 256 & NED01 & 01851.025 & -102234.62 & $14^{\prime \prime} 33$ & $12^{\prime \prime} 67$ & 7.62 & 6.73 & 140 & 1 & $120 \pm 11$ \\
\hline Arp 259 & NED03/04 & 50138.006 & -41529.55 & $27^{\prime \prime} 32$ & $22^{\prime \prime} 31$ & 7.29 & 5.95 & 90 & 1 & $262 \pm 19$ \\
\hline Arp 261 & NED01 & 144931.021 & -101032.23 & $46^{\prime \prime} 16$ & 32.49 & 6.49 & 4.57 & 250 & 1 & $110 \pm 19$ \\
\hline
\end{tabular}


TABLE 3-Continued

\begin{tabular}{|c|c|c|c|c|c|c|c|c|c|c|}
\hline Name & $\begin{array}{l}\text { Individual } \\
\text { Galaxy }\end{array}$ & $\begin{array}{c}\mathrm{RA} \\
(\mathrm{J} 2000)\end{array}$ & $\begin{array}{c}\text { DEC } \\
(\mathrm{J} 2000)\end{array}$ & $\begin{array}{c}\text { Major } \\
\text { Axis } \\
\text { Radius } \\
\left({ }^{\prime \prime}\right)\end{array}$ & $\begin{array}{c}\text { Minor } \\
\text { Axis } \\
\text { Radius } \\
\quad\left({ }^{\prime \prime}\right)\end{array}$ & $\begin{array}{l}\text { Major } \\
\text { Axis } \\
\text { Radius } \\
\text { (kpc) }\end{array}$ & $\begin{array}{c}\text { Minor } \\
\text { Axis } \\
\text { Radius } \\
\text { (kpc) }\end{array}$ & $\begin{array}{l}\text { P.A. } \\
(\mathrm{deg})\end{array}$ & $\begin{array}{l}\text { Number } \\
\text { Annuli }\end{array}$ & $\begin{array}{c}\text { Diffuse } \\
0.3- \\
1.0 \mathrm{keV} \\
\text { Counts }\end{array}$ \\
\hline \multirow[t]{2}{*}{ Arp 270} & NGC 3395 & 104950.028 & +325855.07 & $36^{\prime \prime} .54$ & $27^{\prime \prime} .19$ & 5.14 & 3.82 & 315 & 7 & $274 \pm 22$ \\
\hline & NGC 3396 & 104955.286 & +325926.47 & $17^{\prime \prime} \cdot 91$ & $6^{\prime \prime} 19$ & 2.52 & 0.85 & 160 & 1 & $135 \pm 12$ \\
\hline Arp 283 & NGC 2798 & 91722.830 & +415959.55 & $17^{\prime \prime} .55$ & $15^{\prime \prime} 13$ & 2.55 & 2.20 & 345 & 1 & $65 \pm 9$ \\
\hline Arp 284 & NGC 7714 & 233614.310 & +20914.05 & $35^{\prime \prime} 65$ & $16^{\prime \prime} 66$ & 6.74 & 3.15 & 349 & 13 & $971 \pm 33$ \\
\hline Arp 293 & NGC 6286 & 165831.581 & +585615.77 & $28^{\prime \prime} .09$ & $19^{\prime \prime} 71$ & 11.17 & 7.84 & 231 & 7 & $260 \pm 17$ \\
\hline Arp 295 & ARP295B & 234200.805 & -33653.46 & $14^{\prime \prime} \cdot 22$ & $11^{\prime \prime} 23$ & 6.48 & 5.12 & 350 & 1 & $59 \pm 8$ \\
\hline Arp 299 & NGC 3690 & 112832.174 & +583346.26 & $68^{\prime \prime} .30$ & $45^{\prime \prime} .99$ & 15.90 & 10.71 & 330 & 13 & $7161 \pm 90$ \\
\hline IRAS 17208-0014 & IRAS F17207-0014 & 172321.953 & -0 1701.90 & $17^{\prime \prime} 49$ & $9^{\prime \prime} 52$ & 15.52 & 8.45 & 15 & 5 & $115 \pm 13$ \\
\hline IRAS 23128-5919 & ESO 148-IG002 & 231546.922 & -590314.16 & $16^{\prime \prime} 25$ & $12^{\prime \prime} 74$ & 14.50 & 11.37 & 260 & 13 & $220 \pm 16$ \\
\hline Mrk 231 & UGC 08058 & 125614.547 & +565224.67 & 20.09 & $17^{\prime \prime} 68$ & 17.35 & 15.27 & 105 & 13 & $3349 \pm 63$ \\
\hline Mrk 273 & UGC 08696 & 134442.002 & +555312.22 & $49^{\prime \prime} 60$ & $24^{\prime \prime} .25$ & 38.61 & 18.88 & 100 & 13 & $1121 \pm 36$ \\
\hline NGC 034 & NGC 17 & 01106.714 & -120627.79 & $20^{\prime \prime} 74$ & $20 . \prime 02$ & 7.98 & 7.70 & 120 & 1 & $61 \pm 8$ \\
\hline NGC 1700 & MGC-01-13-038 & 045656.452 & -045159.57 & $622^{\prime \prime} 77$ & $44^{\prime \prime} \cdot 22$ & 15.98 & 11.26 & 20 & 13 & $1714 \pm 49$ \\
\hline NGC $2207 /$ IC 2163 & NGC $2207 /$ IC 2163 & 061622.134 & -212221.18 & $66^{\prime \prime} 36$ & $38^{\prime \prime} .06$ & 12.23 & 7.01 & 15 & 13 & $786 \pm 34$ \\
\hline NGC 2865 & ESO 498-G001 & 92330.226 & -230944.13 & $21^{\prime \prime} .50$ & $12^{\prime \prime} 20$ & 3.95 & 2.24 & 240 & 1 & $75 \pm 10$ \\
\hline NGC 3256 & NGC $3256 \mathrm{~N} / \mathrm{S}$ & 102751.096 & -435409.37 & $41^{\prime \prime} \cdot 22$ & $39 \prime .40$ & 7.40 & 7.07 & 0 & 13 & $5702 \pm 80$ \\
\hline NGC 3353 & UGC 05860 & 104522.210 & +555735.61 & $38^{\prime \prime} 14$ & $27^{\prime \prime} \cdot 79$ & 3.42 & 2.49 & 357 & 1 & $50 \pm 10$ \\
\hline NGC 5018 & UGCA 335 & 131301.040 & -193104.39 & $23^{\prime \prime} 09$ & $13^{\prime \prime} 44$ & 4.30 & 2.50 & 5 & 7 & $283 \pm 18$ \\
\hline NGC 5256 & NED01/02 & 133818.085 & +481638.52 & 40.00 & 32.57 & 23.45 & 19.10 & 90 & 13 & $637 \pm 27$ \\
\hline NGC 6240 & UGC 10592 & 165258.527 & +22404.36 & $48^{\prime \prime} .65$ & $33^{\prime \prime} 88$ & 25.67 & 17.88 & 120 & 13 & $10518 \pm 112$ \\
\hline NGC 7592 & NGC $7592 \mathrm{~A} / \mathrm{B}$ & 231822.276 & -42457.89 & $23^{\prime \prime} 67$ & $22^{\prime \prime} 16$ & 11.42 & 10.70 & 315 & 5 & $126 \pm 12$ \\
\hline UGC 5101 & CGCG 289-001 & 93551.539 & +612111.26 & $24^{\prime \prime} 01$ & $14^{\prime \prime} 18$ & 19.13 & 11.30 & 90 & 1 & $134 \pm 13$ \\
\hline \multirow[t]{2}{*}{ UGC 5189} & UGC 5189 NED01 & 94253.329 & +92938.77 & $10^{\prime \prime} 48$ & $10^{\prime \prime} 40$ & 2.49 & 2.47 & 5 & 1 & $179 \pm 15$ \\
\hline & UGC 5189 NED02 & 94256.660 & +92817.30 & $20{ }^{\prime \prime} 39$ & $4 . \prime 40$ & 4.84 & 1.11 & 45 & 1 & $57 \pm 10$ \\
\hline
\end{tabular}


${ }^{1}$ The position angle of the major axis defined south of east as in the $d s 9$ software. 
TABLE 4

Outer $(2 \sigma)$ Ellipses for Galaxies with high S/N Observations

\begin{tabular}{|c|c|c|c|c|c|c|c|c|c|}
\hline Name & $\begin{array}{c}\mathrm{RA} \\
(\mathrm{J} 2000)\end{array}$ & $\begin{array}{c}\text { DEC } \\
(\mathrm{J} 2000)\end{array}$ & $\begin{array}{c}\text { Major } \\
\text { Axis } \\
\text { Radius } \\
\quad\left({ }^{\prime \prime}\right)\end{array}$ & $\begin{array}{c}\text { Minor } \\
\text { Axis } \\
\text { Radius } \\
\quad\left({ }^{\prime \prime}\right)\end{array}$ & $\begin{array}{c}\text { Major } \\
\text { Axis } \\
\text { Radius } \\
\text { (kpc) }\end{array}$ & $\begin{array}{c}\text { Minor } \\
\text { Axis } \\
\text { Radius } \\
\text { (kpc) }\end{array}$ & $\begin{array}{l}\text { P.A. }{ }^{1} \\
(\mathrm{deg})\end{array}$ & $\begin{array}{c}\text { Diffuse } \\
0.3- \\
1.0 \mathrm{keV} \\
\text { Counts }\end{array}$ & $\begin{array}{c}0.3-1.0 \mathrm{keV} \\
\text { Surface } \\
\text { Brightness } \\
\left(\times 10^{-10}\right. \\
\text { Photons s}^{-1} \\
\left.\mathrm{~cm}^{-2} \operatorname{arcsec}^{-2}\right)\end{array}$ \\
\hline AM 2055-425 & 205826.554 & -423858.11 & $30^{\prime \prime} \cdot 20$ & $13^{\prime \prime} 76$ & 26.23 & 11.96 & 65 & $277 \pm 19$ & $\leq 36.0$ \\
\hline Arp 091 & 153432.396 & +151136.55 & $15^{\prime \prime} 42$ & 9.'90 & 2.54 & 1.63 & 300 & $233 \pm 17$ & $\leq 116.0$ \\
\hline Arp 148 & 110353.839 & +405058.26 & $23^{\prime \prime} \cdot 24$ & $13^{\prime \prime} 43$ & 16.56 & 9.57 & 350 & $267 \pm 18$ & $\leq 49.8$ \\
\hline Arp 155 & 112338.541 & +535030.51 & $41^{\prime \prime} 45$ & $21^{\prime \prime} 08$ & 9.25 & 4.70 & 350 & $167 \pm 19$ & $\leq 19.9$ \\
\hline Arp 157 & 12434.398 & +34727.11 & $75^{\prime \prime} 10$ & $24^{\prime \prime} 96$ & 11.11 & 3.69 & 100 & $349 \pm 27$ & $\leq 22.0$ \\
\hline Arp 160 & 121409.490 & +543132.96 & $49^{\prime \prime} 41$ & $35^{\prime \prime} 13$ & 9.35 & 6.64 & 258 & $1086 \pm 36$ & $\leq 24.7$ \\
\hline Arp 217 & 103845.964 & +533008.73 & $99^{\prime \prime} 33$ & $89^{\prime \prime} 22$ & 8.67 & 7.79 & 230 & $5270 \pm 80$ & $\leq 10.9$ \\
\hline Arp 220 & 153457.578 & +233006.07 & $54^{\prime \prime} 70$ & $38^{\prime \prime} .34$ & 22.02 & 15.44 & 15 & $851 \pm 38$ & $\leq 16.6$ \\
\hline Arp 222 & 233953.876 & -121734.61 & $46^{\prime \prime} 63$ & $27^{\prime \prime} .55$ & 5.90 & 3.49 & 40 & $128 \pm 16$ & $\leq 33.5$ \\
\hline Arp 226 & 222044.774 & -244041.77 & $12^{\prime \prime} 13$ & $9^{\prime \prime} 67$ & 3.94 & 3.14 & 30 & $217 \pm 16$ & $\leq 106.6$ \\
\hline Arp 236 & 010746.897 & -173026.91 & $28^{\prime \prime} .52$ & $28^{\prime \prime} 43$ & 11.20 & 11.17 & 90 & $1652 \pm 42$ & $\leq 18.1$ \\
\hline Arp 244 & 120153.451 & -185226.50 & $143^{\prime \prime} .52$ & $112^{\prime \prime} 32$ & 16.78 & 13.13 & 50 & $55121 \pm 292$ & $\leq 2.4$ \\
\hline Arp 270 & 104950.028 & +325855.07 & $42^{\prime \prime} 63$ & $31^{\prime \prime} 73$ & 60 & 4.46 & 315 & $319 \pm 24$ & $\leq \overline{3} 2.4$ \\
\hline Arp 284 & 233614.310 & +20914.05 & $57 ! 93$ & $27 !$ ! 07 & 10.96 & 5.12 & 349 & $1024 \pm 36$ & $\leq 25.8$ \\
\hline Arp 293 & 165831.581 & +585615.77 & $32^{\prime \prime} 78$ & $22^{\prime \prime} 99$ & 13.03 & 9.14 & 231 & $262 \pm 18$ & $\leq 59.6$ \\
\hline Arp 299 & 112832.174 & +583346.26 & $80^{\prime \prime} 72$ & $54^{\prime \prime} 35$ & 18.79 & 12.65 & 330 & $7434 \pm 94$ & $\leq 13.0$ \\
\hline IRAS 17208-0014 & 172321.953 & -0 1701.90 & $23^{\prime \prime} \cdot 32$ & $12^{\prime \prime} 69$ & 20.69 & 11.27 & 15 & $101 \pm 15$ & $\leq 29.4$ \\
\hline IRAS 23128-5919 & 231546.922 & -590314.16 & $11^{\prime \prime} 61$ & $9^{\prime \prime} 10$ & 10.36 & 8.12 & 260 & $214 \pm 16$ & $\leq 55.7$ \\
\hline Mrk 231 & 125614.547 & +565224.67 & $65^{\prime \prime} \cdot 29$ & $57 ! ! 46$ & 56.40 & 49.64 & 105 & $5375 \pm 109$ & $\leq 8.6$ \\
\hline Mrk 273 & 134442.002 & +555312.22 & $64 . .48$ & $31^{\prime \prime} 53$ & 50.19 & 24.54 & 100 & $1212 \pm 38$ & $\leq 18.8$ \\
\hline NGC 1700 & 045656.452 & -045159.57 & $102^{\prime \prime} .00$ & $71^{\prime \prime} 86$ & 25.97 & 18.30 & 20 & $2090 \pm 62$ & $\leq 11.9$ \\
\hline NGC $2207 /$ IC 2163 & 061622.134 & -212221.18 & $119^{\prime \prime} 45$ & $68 . .50$ & 22.01 & 12.63 & 15 & $1401 \pm 52$ & $\leq 12.0$ \\
\hline NGC 3256 & 102751.096 & -435409.37 & $54^{\prime \prime} .95$ & 52.53 & 9.86 & 9.43 & 0 & $5918 \pm 84$ & $\leq 16.6$ \\
\hline NGC 5018 & 131301.040 & -193104.39 & $233^{\prime \prime} 09$ & 13 ! 44 & 4.30 & 2.50 & 5 & $283 \pm 18$ & $\leq 53.9$ \\
\hline NGC 5256 & 133818.085 & +481638.52 & $51^{\prime \prime} 99$ & $42^{\prime \prime} \cdot 34$ & 30.49 & 24.82 & 90 & $672 \pm 29$ & $\leq 29.9$ \\
\hline
\end{tabular}


TABLE 4 -Continued

\begin{tabular}{|c|c|c|c|c|c|c|c|c|c|}
\hline Name & $\begin{array}{c}\mathrm{RA} \\
(\mathrm{J} 2000)\end{array}$ & $\begin{array}{c}\text { DEC } \\
(\mathrm{J} 2000)\end{array}$ & $\begin{array}{c}\text { Major } \\
\text { Axis } \\
\text { Radius } \\
\left({ }^{\prime \prime}\right)\end{array}$ & $\begin{array}{c}\text { Minor } \\
\text { Axis } \\
\text { Radius } \\
\left({ }^{\prime \prime}\right)\end{array}$ & $\begin{array}{l}\text { Major } \\
\text { Axis } \\
\text { Radius } \\
\text { (kpc) }\end{array}$ & $\begin{array}{l}\text { Minor } \\
\text { Axis } \\
\text { Radius } \\
(\mathrm{kpc})\end{array}$ & $\begin{array}{l}\text { P.A. }{ }^{1} \\
\text { (deg) }\end{array}$ & $\begin{array}{c}\text { Diffuse } \\
0.3- \\
1.0 \mathrm{keV} \\
\text { Counts }\end{array}$ & $\begin{array}{c}0.3-1.0 \mathrm{keV} \\
\text { Surface } \\
\text { Brightness } \\
\left(\times 10^{-10}\right. \\
\text { Photons s}^{-1} \\
\left.\mathrm{~cm}^{-2} \operatorname{arcsec}^{-2}\right)\end{array}$ \\
\hline NGC 6240 & 165258.527 & +2 2404.36 & $90^{\prime \prime} 35$ & $62^{\prime \prime} .91$ & 47.67 & 33.20 & 120 & $12936 \pm 141$ & $\leq 8.3$ \\
\hline NGC 7592 & 231822.276 & -42457.89 & $31^{\prime \prime} .56$ & $29 \prime .55$ & 15.23 & 14.26 & 315 & $124 \pm 13$ & $\leq 37.1$ \\
\hline
\end{tabular}

${ }^{1}$ The position angle of the major axis defined south of east as in the $d s 9$ software. 
TABLE 5

Correlations and Anti-Correlations

\begin{tabular}{|c|c|c|c|c|c|c|}
\hline $\begin{array}{l}\text { Fig } \\
\text { Num }\end{array}$ & $\begin{array}{c}\text { SFR } \\
\text { Range }\end{array}$ & $\begin{array}{c}\mathrm{CO} / \mathrm{H}_{2} \\
\text { Ratio }\end{array}$ & Relation & $\mathrm{rms}$ & $\begin{array}{c}\text { Spear/ } \\
\text { Pearson } \\
\text { Coeff. }\end{array}$ & Correl. \\
\hline \multicolumn{7}{|c|}{ Basic Relations } \\
\hline 2 & all & & $\mathrm{LOG} \mathrm{SFR}=(0.79 \pm 0.21) \mathrm{LOG} \mathrm{L}_{\mathrm{K}}-(7.75 \pm 2.29)$ & 0.76 & $0.47 / 0.51$ & weak \\
\hline 2 & $>1 \frac{M_{\odot}}{y r}$ & & LOG SFR $=(0.84 \pm 0.19)$ LOG $\mathrm{L}_{K}-(8.05 \pm 2.09)$ & 0.48 & $0.51 / 0.62$ & weak \\
\hline 2 & $y r$ & & $\mathrm{LOG}$ SFR $=(0.88 \pm 0.11) \mathrm{LOG}\left(\mathrm{L}_{\mathrm{FIR}} / \mathrm{L}_{\mathrm{K}}\right)+(1.33 \pm 0.1)$ & 0.56 & $0.79 / 0.77$ & strong \\
\hline 2 & $>1 \frac{M_{\odot}}{y r}$ & & $\mathrm{LOG} S \mathrm{SFR}=(0.95 \pm 0.16) \mathrm{LOG}\left(\mathrm{L}_{\mathrm{FIR}} / \mathrm{L}_{\mathrm{K}}\right)+(1.46 \pm 0.08)$ & 0.42 & $0.75 / 0.72$ & strong \\
\hline 2 & $y r$ & & LOG SFR $=(0.41 \pm 0.05)([3.6]-[24])-(1.74 \pm 0.33)$ & 0.53 & $0.81 / 0.80$ & strong \\
\hline 2 & $>1 \frac{M_{\odot}}{y r}$ & & LOG SFR $=(0.41 \pm 0.07)([3.6]-[24])-(1.62 \pm 0.5)$ & 0.43 & $0.76 / 0.72$ & strong \\
\hline 2 & & & LOG SFR $=(3.46 \pm 0.76)$ LOG $\left(\mathrm{F}_{60} / \mathrm{F}_{100}\right)+(1.49 \pm 0.15)$ & 0.70 & $0.60 / 0.58$ & strong \\
\hline 2 & $>1 \frac{M_{\odot}}{y r}$ & & LOG SFR $=(2.17 \pm 0.69)$ LOG $\left(\mathrm{F}_{60} / \mathrm{F}_{100}\right)+(1.55 \pm 0.12)$ & 0.54 & $0.50 / 0.48$ & weak \\
\hline 4 & all & Const & LOG SFE $=(2.54 \pm 0.47)$ LOG $\left(\mathrm{F}_{60} / \mathrm{F}_{100}\right)-(8.12 \pm 0.09)$ & 0.42 & $0.72 / 0.67$ & strong \\
\hline 4 & $>1 \frac{M_{\odot}}{y r}$ & Const & $\mathrm{LOG} S \mathrm{SFE}=(2.93 \pm 0.48)$ LOG $\left(\mathrm{F}_{60} / \mathrm{F}_{100}\right)-(8.13 \pm 0.08)$ & 0.37 & $0.76 / 0.74$ & strong \\
\hline 4 & & Var & LOG SFE $=(3.38 \pm 0.55)$ LOG $\left(\mathrm{F}_{60} / \mathrm{F}_{100}\right)-(7.68 \pm 0.11)$ & 0.49 & $0.73 / 0.72$ & strong \\
\hline 4 & $>1 \frac{M_{\odot}}{y r}$ & Var & LOG SFE $=(3.60 \pm 0.63)$ LOG $\left(\mathrm{F}_{60} / \mathrm{F}_{100}\right)-(7.66 \pm 0.11)$ & 0.49 & $0.71 / 0.71$ & strong \\
\hline 4 & all & Const & LOG SFE $=(0.23 \pm 0.11)$ LOG SFR $-(8.72 \pm 0.16)$ & 0.53 & $0.40 / 0.33$ & weak \\
\hline 4 & $>1 \frac{M_{\odot}}{y r}$ & Const & LOG SFE $=(0.37 \pm 0.14)$ LOG SFR $-(8.95 \pm 0.21)$ & 0.50 & $0.44 / 0.42$ & weak \\
\hline 4 & all & Var & LOG SFE $=(0.61 \pm 0.10)$ LOG SFR $-(8.82 \pm 0.15)$ & 0.50 & $0.75 / 0.71$ & strong \\
\hline 4 & $>1 \frac{M_{\odot}}{y r}$ & Var & LOG SFE $=(0.88 \pm 0.13)$ LOG SFR $-(9.24 \pm 0.19)$ & 0.43 & $0.77 / 0.78$ & strong \\
\hline \multicolumn{7}{|c|}{ Comparisons with Volume } \\
\hline 9 & all & & LOG VOLUME $=(0.66 \pm 0.10)$ LOG SFR $+(67.01 \pm 0.14)$ & 0.59 & $0.75 / 0.70$ & strong \\
\hline 9 & $>1 \frac{M_{\odot}}{y r}$ & & LOG VOLUME $=(0.97 \pm 0.15)$ LOG SFR $+(66.54 \pm 0.22)$ & 0.52 & $0.79 / 0.75$ & strong \\
\hline 10 & all & Const & LOG VOLUME vs. LOG SFE & 0.85 & $0.16 / 0.06$ & none \\
\hline 10 & all & Var & LOG VOLUME $=(0.57 \pm 0.18)$ LOG SFE $+(72.36 \pm 1.48)$ & 0.75 & $0.53 / 0.47$ & weak \\
\hline 10 & all & & LOG VOLUME vs. LOG $\left(\mathrm{F}_{60} / \mathrm{F}_{100}\right)$ & 0.79 & $0.34 / 0.29$ & none \\
\hline 10 & $>1 \frac{M_{\odot}}{y r}$ & & LOG VOLUME vs. LOG $\left(\mathrm{F}_{60} / \mathrm{F}_{100}\right)$ & 0.78 & $0.20 / 0.11$ & none \\
\hline 11 & all & & LOG VOLUME $=(0.80 \pm 0.12)$ LOG $L_{X}($ gas $)+(35.11 \pm 4.85)$ & 0.57 & $0.79 / 0.72$ & strong \\
\hline 11 & all & & LOG VOLUME $=(-1.19 \pm 0.39)$ LOG $n_{e}+(64.95 \pm 0.9)$ & 0.75 & $-0.39 /-0.43$ & weak anti \\
\hline 11 & all & & LOG VOLUME $=(0.85 \pm 0.18)$ LOG $L_{K}+(58.23 \pm 2.01)$ & 0.67 & $0.65 / 0.59$ & strong \\
\hline 11 & $>1 \frac{M_{\odot}}{u r}$ & & LOG VOLUME $=(1.15 \pm 0.23)$ LOG $\mathrm{L}_{K}+(54.92 \pm 2.58)$ & 0.60 & $0.65 / 0.66$ & strong \\
\hline 11 & all & & LOG VOLUME $=(0.40 \pm 0.15)$ LOG $\left(\mathrm{L}_{\mathrm{FIR}} / \mathrm{L}_{\mathrm{K}}\right)+(67.81 \pm 0.13)$ & 0.77 & $0.49 / 0.37$ & weak \\
\hline none & all & & LOG VOLUME $=(0.17 \pm 0.07)([3.6]-[24])+(66.5 \pm 0.48)$ & 0.77 & $0.44 / 0.36$ & weak \\
\hline none & all & & LOG (VOLUME/SFR) vs. LOG SFR & 0.59 & $-0.34 /-0.46$ & none \\
\hline none & $>1 \frac{M_{\odot}}{y r}$ & & LOG (VOLUME/SFR) vs. LOG SFR & 0.52 & $0.02 /-0.04$ & none \\
\hline none & & & LOG $\left(\right.$ VOLUME $\left./ \mathrm{L}_{K}\right)=(0.32 \pm 0.11)$ LOG SFR $+(56.27 \pm 0.14)$ & 0.61 & $0.40 / 0.42$ & weak \\
\hline none & $>1 \frac{M_{\odot}}{y r}$ & & LOG $\left(\right.$ VOLUME $\left./ \mathrm{L}_{K}\right)=(0.52 \pm 0.15)$ LOG SFR $+(55.97 \pm 0.21)$ & 0.51 & $0.51 / 0.53$ & weak \\
\hline none & $y r$ & & LOG $($ VOLUME/LK $)$ vs. LOG $L_{K}$ & 0.67 & $0.02 /-0.13$ & none \\
\hline none & $>1 \frac{M_{\odot}}{y r}$ & & LOG (VOLUME/LK $)$ vs. LOG $\mathrm{L}_{K}$ & 0.60 & $0.16 / 0.12$ & none \\
\hline \multicolumn{7}{|c|}{ Comparisons with LOG (LX (gas)/SFR) } \\
\hline none & all & & LOG $\left(\mathrm{L}_{\mathrm{X}}(\mathrm{gas}) / \mathrm{SFR}\right)$ vs. LOG SFR & 0.40 & $-0.35 /-0.53$ & none \\
\hline none & $>1 \frac{M_{\odot}}{u r}$ & & LOG $\left(\mathrm{L}_{X}\right.$ (gas)/SFR) vs. LOG SFR & 0.30 & $-0.35 /-0.48$ & none \\
\hline none & all & & $\mathrm{LOG}\left(\mathrm{L}_{\mathrm{X}}(\mathrm{gas}) / \mathrm{SFR}\right)=(-1.69 \pm 0.37) \mathrm{LOG}\left(\mathrm{F}_{60} / \mathrm{F}_{100}\right)+(39.6 \pm 0.07)$ & 0.34 & $-0.53 /-0.58$ & weak anti \\
\hline none & $>1 \frac{M_{\odot}}{y r}$ & & $\mathrm{LOG}\left(\mathrm{L}_{\mathrm{X}}(\mathrm{gas}) / \mathrm{SFR}\right)=(-1.28 \pm 0.38) \mathrm{LOG}\left(\mathrm{F}_{60} / \mathrm{F}_{100}\right)+(39.64 \pm 0.07)$ & 0.30 & $-0.45 /-0.50$ & weak anti \\
\hline none & all & Const & $\mathrm{LOG}\left(\mathrm{Lx}_{\mathrm{X}}(\mathrm{gas}) / \mathrm{SFR}\right)=(-0.40 \pm 0.10) \mathrm{LOG} \mathrm{SFE}+(36.39 \pm 0.81)$ & 0.32 & $-0.55 /-0.58$ & weak anti \\
\hline none & $>1 \frac{M_{\odot}}{y r}$ & Const & LOG $\left(\mathrm{L}_{\mathrm{X}}(\mathrm{gas}) / \mathrm{SFR}\right)=(-0.36 \pm 0.09)$ LOG SFE $+(36.76 \pm 0.8)$ & 0.29 & $-0.49 /-0.56$ & weak anti \\
\hline none & all & Var & $\mathrm{LOG}\left(\mathrm{L}_{\mathrm{X}}(\mathrm{gas}) / \mathrm{SFR}\right)=(-0.32 \pm 0.08) \mathrm{LOG} \mathrm{SFE}+(37.22 \pm 0.63)$ & 0.32 & $-0.55 /-0.57$ & weak anti \\
\hline none & $>1 \frac{M_{\odot}}{y r}$ & Var & $\mathrm{LOG}\left(\mathrm{L}_{\mathrm{X}}(\mathrm{gas}) / \mathrm{SFR}\right)=(-0.27 \pm 0.07) \mathrm{LOG} \mathrm{SFE}+(37.58 \pm 0.61)$ & 0.29 & $-0.52 /-0.55$ & weak anti \\
\hline \multicolumn{7}{|c|}{ Comparisons with LOG $\mathrm{M}_{\mathrm{X}}(\mathrm{gas})$ and $\mathrm{LOG} \mathrm{M}_{\mathrm{X}}$ (gas)/SFR } \\
\hline 13 & all & & LOG $\mathrm{M}_{\mathrm{X}}(\mathrm{gas})=(0.70 \pm 0.07)$ LOG SFR $+(7.6 \pm 0.1)$ & 0.42 & $0.86 / 0.83$ & strong \\
\hline 13 & $>1 \frac{M_{\odot}}{u r}$ & & LOG $\mathrm{M}_{\mathrm{X}}($ gas $)=(0.88 \pm 0.10)$ LOG SFR $+(7.35 \pm 0.14)$ & 0.34 & $0.86 / 0.84$ & strong \\
\hline 13 & all $^{y r}$ & & $\mathrm{LOG} \mathrm{M}_{\mathrm{X}}(\mathrm{gas})=(0.94 \pm 0.14) \mathrm{LOG} \mathrm{L}_{\mathrm{K}}-(2.05 \pm 1.58)$ & 0.53 & $0.69 / 0.72$ & strong \\
\hline 13 & $>1 \frac{M_{\odot}}{y r}$ & & LOG $\mathrm{M}_{\mathrm{X}}($ gas $)=(1.00 \pm 0.17)$ LOG $\mathrm{L}_{\mathrm{K}}-(2.68 \pm 1.94)$ & 0.45 & $0.67 / 0.71$ & strong \\
\hline
\end{tabular}


TABle 5-Continued

\begin{tabular}{|c|c|c|c|c|c|c|}
\hline $\begin{array}{l}\text { Fig } \\
\text { Num }\end{array}$ & $\begin{array}{c}\text { SFR } \\
\text { Range }\end{array}$ & $\begin{array}{c}\mathrm{CO} / \mathrm{H}_{2} \\
\text { Ratio }\end{array}$ & Relation & $\mathrm{rms}$ & $\begin{array}{c}\text { Spear/ } \\
\text { Pearson } \\
\text { Coeff. }\end{array}$ & Correl. \\
\hline 13 & all & & $\mathrm{LOG}\left(\mathrm{M}_{\mathrm{X}}(\right.$ gas $\left.) / \mathrm{SFR}\right)=(-0.30 \pm 0.07) \mathrm{LOG} \mathrm{SFR}+(7.6 \pm 0.1)$ & 0.42 & $-0.38 /-0.53$ & weak anti \\
\hline 13 & $>1 \frac{M_{\odot}}{y r}$ & & LOG $\left(\mathrm{M}_{\mathrm{X}}(\mathrm{gas}) / \mathrm{SFR}\right)$ vs. LOG SFR & 0.34 & $-0.12 /-0.22$ & none \\
\hline 13 & all & & LOG $\left(\mathrm{M}_{\mathrm{X}}(\right.$ gas $\left.) / \mathrm{SFR}\right)$ vs. LOG $\mathrm{L}_{\mathrm{K}}$ & 0.49 & $0.28 / 0.17$ & none \\
\hline 13 & $>1 \frac{M_{\odot}}{y r}$ & & $\mathrm{LOG}\left(\mathrm{M}_{\mathrm{X}}(\right.$ gas $\left.) / \mathrm{SFR}\right)=(0.16 \pm 0.13) \mathrm{LOG} \mathrm{L}_{\mathrm{K}}+(5.37 \pm 1.48)$ & 0.34 & $0.37 / 0.21$ & weak \\
\hline 14 & all & Const & $\mathrm{LOG}\left(\mathrm{M}_{\mathrm{X}}(\mathrm{gas}) / \mathrm{SFR}\right)=(-0.37 \pm 0.10) \mathrm{LOG} \mathrm{SFE}+(4.06 \pm 0.84)$ & 0.33 & $-0.52 /-0.54$ & weak anti \\
\hline 14 & $>1 \frac{M_{\odot}}{y r}$ & Const & $\mathrm{LOG}\left(\mathrm{M}_{\mathrm{X}}(\mathrm{gas}) / \mathrm{SFR}\right)=(-0.33 \pm 0.10) \mathrm{LOG} \mathrm{SFE}+(4.38 \pm 0.85)$ & 0.31 & $-0.45 /-0.51$ & weak anti \\
\hline 14 & all & Var & $\mathrm{LOG}\left(\mathrm{M}_{\mathrm{X}}(\mathrm{gas}) / \mathrm{SFR}\right)=(-0.26 \pm 0.08) \mathrm{LOG} \mathrm{SFE}+(5.11 \pm 0.68)$ & 0.35 & $-0.42 /-0.46$ & weak anti \\
\hline 14 & $>1 \frac{M_{\odot}}{y r}$ & Var & LOG $\left(\mathrm{M}_{\mathrm{X}}(\right.$ gas $\left.) / \mathrm{SFR}\right)$ vs. LOG SFE & 0.33 & $-0.34 /-0.39$ & none \\
\hline 14 & & & $\mathrm{LOG}\left(\mathrm{M}_{\mathrm{X}}(\mathrm{gas}) / \mathrm{SFR}\right)=(-0.22 \pm 0.03)([3.6]-[24])+(8.78 \pm 0.19)$ & 0.31 & $-0.66 /-0.77$ & strong anti \\
\hline 14 & $>1 \frac{M_{\odot}}{y r}$ & & $\mathrm{LOG}\left(\mathrm{M}_{\mathrm{X}}(\mathrm{gas}) / \mathrm{SFR}\right)=(-0.17 \pm 0.05)([3.6]-[24])+(8.41 \pm 0.35)$ & 0.30 & $-0.50 /-0.52$ & weak anti \\
\hline 14 & all & & $\mathrm{LOG}\left(\mathrm{M}_{\mathrm{X}}(\mathrm{gas}) / \mathrm{SFR}\right)=(-0.45 \pm 0.07) \mathrm{LOG}\left(\mathrm{L}_{\mathrm{FIR}} / \mathrm{L}_{\mathrm{K}}\right)+(7.13 \pm 0.06)$ & 0.35 & $-0.51 /-0.71$ & weak anti \\
\hline 14 & $>1 \frac{M_{\odot}}{y r}$ & & LOG $\left(\mathrm{M}_{\mathrm{X}}(\mathrm{gas}) / \mathrm{SFR}\right)$ vs. LOG $\left(\mathrm{L}_{\mathrm{FIR}} / \mathrm{L}_{\mathrm{K}}\right)$ & 0.33 & $-0.28 /-0.33$ & none \\
\hline 14 & all & & $\operatorname{LOG}\left(\mathrm{M}_{\mathrm{X}}(\right.$ gas $\left.) / \mathrm{SFR}\right)=(-1.66 \pm 0.34) \mathrm{LOG}\left(\mathrm{F}_{60} / \mathrm{F}_{100}\right)+(7.04 \pm 0.07)$ & 0.31 & $-0.59 /-0.61$ & strong anti \\
\hline 14 & $>1 \frac{M_{\odot}}{u r}$ & & $\operatorname{LOG}\left(\mathrm{M}_{\mathrm{X}}(\right.$ gas $\left.) / \mathrm{SFR}\right)=(-1.33 \pm 0.38) \mathrm{LOG}\left(\mathrm{F}_{60} / \mathrm{F}_{100}\right)+(7.04 \pm 0.07)$ & 0.30 & $-0.48 /-0.51$ & weak anti \\
\hline 14 & all & & LOG $\left(\mathrm{M}_{\mathrm{X}}(\right.$ gas $\left.) / \mathrm{SFR}\right)$ vs. LOG $\mathrm{n}_{\mathrm{e}}$ & 0.46 & $-0.34 /-0.38$ & none \\
\hline 14 & $>1 \frac{M_{\odot}}{y r}$ & & $\mathrm{LOG}\left(\mathrm{M}_{\mathrm{X}}(\mathrm{gas}) / \mathrm{SFR}\right)=(-0.55 \pm 0.20) \mathrm{LOG} \mathrm{n}_{\mathrm{e}}+(5.97 \pm 0.46)$ & 0.32 & $-0.39 /-0.42$ & weak anti \\
\hline none & all & & $\mathrm{LOG}\left(\mathrm{M}_{\mathrm{X}}(\right.$ gas $\left.) / \mathrm{L}_{\mathrm{K}}\right)=(0.53 \pm 0.07) \mathrm{LOG}\left(\mathrm{SFR} / \mathrm{L}_{\mathrm{K}}\right)+(2.54 \pm 0.68)$ & 0.33 & $0.77 / 0.77$ & strong \\
\hline none & $>1 \frac{M_{\odot}}{y r}$ & & $\mathrm{LOG}\left(\mathrm{M}_{\mathrm{X}}(\mathrm{gas}) / \mathrm{L}_{\mathrm{K}}\right)=(0.67 \pm 0.11) \mathrm{LOG}\left(\mathrm{SFR} / \mathrm{L}_{\mathrm{K}}\right)+(3.91 \pm 1.09)$ & 0.31 & $0.73 / 0.73$ & strong \\
\hline none & all & & $\operatorname{LOG}\left(\mathrm{M}_{\mathrm{X}}(\mathrm{gas}) / \mathrm{L}_{\mathrm{K}}\right)=(0.37 \pm 0.07) \mathrm{LOG}$ SFR $-(3.13 \pm 0.1)$ & 0.41 & $0.63 / 0.73$ & strong \\
\hline none & $>1 \frac{M_{\odot}}{u r}$ & & $\mathrm{LOG}\left(\mathrm{M}_{\mathrm{X}}(\mathrm{gas}) / \mathrm{L}_{\mathrm{K}}\right)=(0.42 \pm 0.10) \mathrm{LOG} \mathrm{SFR}-(3.22 \pm 0.15)$ & 0.37 & $0.62 / 0.73$ & strong \\
\hline none & & & LOG $\left(\mathrm{M}_{\mathrm{X}}(\right.$ gas $\left.) / \mathrm{L}_{\mathrm{K}}\right)$ vs. LOG $\mathrm{L}_{\mathrm{K}}$ & 0.52 & $0.01 /-0.07$ & none \\
\hline none & $>1 \frac{M_{\odot}}{y r}$ & & LOG $\left(\mathrm{M}_{\mathrm{X}}(\right.$ gas $\left.) / \mathrm{L}_{\mathrm{K}}\right)$ vs. LOG $\mathrm{L}_{\mathrm{K}}$ & 0.45 & $0.04 / 0.00$ & none \\
\hline none & all & & $\mathrm{LOG}\left(\mathrm{M}_{\mathrm{X}}(\mathrm{gas}) / \mathrm{SFR}\right)=(0.47 \pm 0.07) \mathrm{LOG}\left(\mathrm{L}_{\mathrm{K}} / \mathrm{SFR}\right)+(2.54 \pm 0.68)$ & 0.33 & $0.58 / 0.74$ & strong \\
\hline none & $>1 \frac{M_{\odot}}{u r}$ & & $\mathrm{LOG}\left(\mathrm{M}_{\mathrm{X}}(\mathrm{gas}) / \mathrm{SFR}\right)=(0.33 \pm 0.11) \mathrm{LOG}\left(\mathrm{L}_{\mathrm{K}} / \mathrm{SFR}\right)+(3.91 \pm 1.09)$ & 0.31 & $0.41 / 0.46$ & weak \\
\hline none & all & Const & LOG $\left(\mathrm{M}_{\mathrm{X}}(\right.$ gas $\left.) / \mathrm{SFR}\right)$ vs. LOG $\left(\mathrm{M}_{\mathrm{HI}} / \mathrm{M}_{\mathrm{H}_{2}}\right)$ & 0.48 & $-0.30 /-0.27$ & none \\
\hline none & all & Var & LOG $\left(\mathrm{M}_{\mathrm{X}}(\right.$ gas $\left.) / \mathrm{SFR}\right)$ vs. LOG $\left(\mathrm{M}_{\mathrm{HI}} / \mathrm{M}_{\mathrm{H}_{2}}\right)$ & 0.48 & $-0.31 /-0.27$ & none \\
\hline none & all & & LOG $\left(\mathrm{M}_{\mathrm{X}}\right.$ (gas)/SFR) vs. LOG VOLUME & 0.49 & $0.17 / 0.16$ & none \\
\hline \multicolumn{7}{|c|}{ Comparisons with LOG $\left(\mathrm{M}_{\mathrm{X}}(\mathrm{gas}) /\left(\mathrm{M}_{\mathrm{H}_{2}}+\mathrm{M}_{\mathrm{HI}}\right)\right)$} \\
\hline 15 & all & Const & $\operatorname{LOG}\left(\mathrm{M}_{\mathrm{X}}(\right.$ gas $\left.) /\left(\mathrm{M}_{\mathrm{H}_{2}}+\mathrm{M}_{\mathrm{HI}}\right)\right)=(0.25 \pm 0.10) \mathrm{LOG} \mathrm{SFR}-(2.05 \pm 0.13)$ & 0.41 & $0.50 / 0.42$ & weak \\
\hline 15 & $>1 \frac{M_{\odot}}{y r}$ & Const & $\operatorname{LOG}\left(\mathrm{M}_{\mathrm{X}}(\right.$ gas $\left.) /\left(\mathrm{M}_{\mathrm{H}_{2}}+\mathrm{M}_{\mathrm{HI}}\right)\right)=(0.58 \pm 0.14) \mathrm{LOG} \mathrm{SFR}-(2.49 \pm 0.19)$ & 0.37 & $0.71 / 0.65$ & strong \\
\hline 15 & all & Var & $\operatorname{LOG}\left(\mathrm{M}_{\mathrm{X}}(\right.$ gas $\left.) /\left(\mathrm{M}_{\mathrm{H}_{2}}+\mathrm{M}_{\mathrm{HI}}\right)\right)=(0.40 \pm 0.12) \mathrm{LOG}$ SFR $-(2.07 \pm 0.15)$ & 0.48 & $0.65 / 0.54$ & strong \\
\hline 15 & $>1 \frac{M_{\odot}}{y r}$ & Var & $\operatorname{LOG}\left(\mathrm{M}_{\mathrm{X}}(\right.$ gas $\left.) /\left(\mathrm{M}_{\mathrm{H}_{2}}+\mathrm{M}_{\mathrm{HI}}\right)\right)=(0.82 \pm 0.16) \mathrm{LOG} \mathrm{SFR}-(2.64 \pm 0.21)$ & 0.41 & $0.80 / 0.73$ & strong \\
\hline 15 & all & Const & $\operatorname{LOG}\left(\mathrm{M}_{\mathrm{X}}(\right.$ gas $\left.) /\left(\mathrm{M}_{\mathrm{H}_{2}}+\mathrm{M}_{\mathrm{HI}}\right)\right)$ vs. LOG SFE & 0.43 & $0.35 / 0.32$ & none \\
\hline 15 & $>1 \frac{M_{\odot}}{y r}$ & Const & $\mathrm{LOG}\left(\mathrm{M}_{\mathrm{X}}(\right.$ gas $\left.) /\left(\mathrm{M}_{\mathrm{H}_{2}}+\mathrm{M}_{\mathrm{HI}}\right)\right)=(0.35 \pm 0.17) \mathrm{LOG} \mathrm{SFE}+(1.21 \pm 1.45)$ & 0.45 & $0.48 / 0.40$ & weak \\
\hline 15 & all ${ }^{y r}$ & Var & LOG $\left(\mathrm{M}_{\mathrm{X}}(\right.$ gas $\left.) /\left(\mathrm{M}_{\mathrm{H}_{2}}+\mathrm{M}_{\mathrm{HI}}\right)\right)=(0.52 \pm 0.13) \mathrm{LOG} \mathrm{SFE}+(2.6 \pm 1.07)$ & 0.45 & $0.61 / 0.61$ & strong \\
\hline 15 & $>1 \frac{M_{\odot}}{y r}$ & Var & $\mathrm{LOG}\left(\mathrm{M}_{\mathrm{X}}(\mathrm{gas}) /\left(\mathrm{M}_{\mathrm{H}_{2}}+\mathrm{M}_{\mathrm{HI}}\right)\right)=(0.60 \pm 0.14) \mathrm{LOG} \mathrm{SFE}+(3.21 \pm 1.16)$ & 0.45 & $0.70 / 0.66$ & strong \\
\hline 16 & all & Const & $\operatorname{LOG}\left(\mathrm{M}_{\mathrm{X}}(\right.$ gas $\left.) /\left(\mathrm{M}_{\mathrm{H}_{2}}+\mathrm{M}_{\mathrm{HI}}\right)\right)$ vs. LOG $\mathrm{L}_{\mathrm{K}}$ & 0.43 & $0.31 / 0.33$ & none \\
\hline 16 & $>1 \frac{M_{\odot}}{y r}$ & Const & LOG $\left(\mathrm{M}_{\mathrm{X}}(\right.$ gas $\left.) /\left(\mathrm{M}_{\mathrm{H}_{2}}+\mathrm{M}_{\mathrm{HI}}\right)\right)$ vs. LOG $\mathrm{L}_{\mathrm{K}}$ & 0.46 & $0.31 / 0.33$ & none \\
\hline 16 & all & Var & $\mathrm{LOG}\left(\mathrm{M}_{\mathrm{X}}(\right.$ gas $\left.) /\left(\mathrm{M}_{\mathrm{H}_{2}}+\mathrm{M}_{\mathrm{HI}}\right)\right)=(0.49 \pm 0.22) \mathrm{LOG} \mathrm{L}_{\mathrm{K}}-(7.12 \pm 2.43)$ & 0.52 & $0.41 / 0.40$ & weak \\
\hline 16 & $>1 \frac{M_{\odot}}{y r}$ & Var & $\mathrm{LOG}\left(\mathrm{M}_{\mathrm{X}}(\right.$ gas $\left.) /\left(\mathrm{M}_{\mathrm{H}_{2}}+\mathrm{M}_{\mathrm{HI}}\right)\right)=(0.60 \pm 0.29) \mathrm{LOG} \mathrm{L}_{\mathrm{K}}-(8.29 \pm 3.23)$ & 0.56 & $0.40 / 0.39$ & weak \\
\hline 16 & all & Const & $\mathrm{LOG}\left(\mathrm{M}_{\mathrm{X}}(\operatorname{gas}) /\left(\mathrm{M}_{\mathrm{H}_{2}}+\mathrm{M}_{\mathrm{HI}}\right)\right)$ vs. LOG $\left(\mathrm{F}_{60} / \mathrm{F}_{100}\right)$ & 0.44 & $0.29 / 0.24$ & none \\
\hline 16 & $>1 \frac{M_{\odot}}{y r}$ & Const & $\operatorname{LOG}\left(\mathrm{M}_{\mathrm{X}}(\right.$ gas $\left.) /\left(\mathrm{M}_{\mathrm{H}_{2}}+\mathrm{M}_{\mathrm{HI}}\right)\right)=(1.02 \pm 0.66) \operatorname{LOG}\left(\mathrm{F}_{60} / \mathrm{F}_{100}\right)-(1.68 \pm 0.12)$ & 0.46 & $0.44 / 0.31$ & weak \\
\hline 16 & all & Var & $\operatorname{LOG}\left(\mathrm{M}_{\mathrm{X}}(\mathrm{gas}) /\left(\mathrm{M}_{\mathrm{H}_{2}}+\mathrm{M}_{\mathrm{HI}}\right)\right)$ vs. LOG $\left(\mathrm{F}_{60} / \mathrm{F}_{100}\right)$ & 0.54 & $0.34 / 0.28$ & none \\
\hline 16 & $>1 \frac{M_{\odot}}{y r}$ & Var & $\operatorname{LOG}\left(\mathrm{M}_{\mathrm{X}}(\right.$ gas $\left.) /\left(\mathrm{M}_{\mathrm{H}_{2}}+\mathrm{M}_{\mathrm{HI}}\right)\right)=(1.32 \pm 0.82) \operatorname{LOG}\left(\mathrm{F}_{60} / \mathrm{F}_{100}\right)-(1.5 \pm 0.15)$ & 0.57 & $0.41 / 0.32$ & weak \\
\hline 17 & all & Const & $\operatorname{LOG}\left(\mathrm{M}_{\mathrm{X}}(\mathrm{gas}) /\left(\mathrm{M}_{\mathrm{H}_{2}}+\mathrm{M}_{\mathrm{HI}}\right)\right)$ vs. $([3.6]-[24])$ & 0.44 & $0.35 / 0.23$ & none \\
\hline 17 & $>1 \frac{M_{\odot}}{y r}$ & Const & $\operatorname{LOG}\left(\mathrm{M}_{\mathrm{X}}(\right.$ gas $\left.) /\left(\mathrm{M}_{\mathrm{H}_{2}}+\mathrm{M}_{\mathrm{HI}}\right)\right)=(0.20 \pm 0.08)([3.6]-[24])-(3.21 \pm 0.61)$ & 0.44 & $0.54 / 0.44$ & weak \\
\hline 17 & all & Var & $\operatorname{LOG}\left(\mathrm{M}_{\mathrm{X}}(\right.$ gas $\left.) /\left(\mathrm{M}_{\mathrm{H}_{2}}+\mathrm{M}_{\mathrm{HI}}\right)\right)=(0.11 \pm 0.07)([3.6]-[24])-(2.44 \pm 0.46)$ & 0.54 & $0.44 / 0.31$ & weak \\
\hline 17 & $>1 \frac{M_{\odot}}{y r}$ & Var & $\operatorname{LOG}\left(\mathrm{M}_{\mathrm{X}}(\right.$ gas $\left.) /\left(\mathrm{M}_{\mathrm{H}_{2}}+\mathrm{M}_{\mathrm{HI}}\right)\right)=(0.26 \pm 0.10)([3.6]-[24])-(3.55 \pm 0.74)$ & 0.53 & $0.55 / 0.47$ & weak \\
\hline 17 & all & Const & $\operatorname{LOG}\left(\mathrm{M}_{\mathrm{X}}(\right.$ gas $\left.) /\left(\mathrm{M}_{\mathrm{H}_{2}}+\mathrm{M}_{\mathrm{HI}}\right)\right)=(0.19 \pm 0.13) \mathrm{LOG}\left(\mathrm{L}_{\mathrm{FIR}} / \mathrm{L}_{\mathrm{K}}\right)-(1.73 \pm 0.1)$ & 0.44 & $0.43 / 0.27$ & weak \\
\hline 17 & $>1 \frac{M_{\odot}}{y r}$ & Const & $\mathrm{LOG}\left(\mathrm{M}_{\mathrm{X}}(\right.$ gas $\left.) /\left(\mathrm{M}_{\mathrm{H}_{2}}+\mathrm{M}_{\mathrm{HI}}\right)\right)=(0.53 \pm 0.20) \mathrm{LOG}\left(\mathrm{L}_{\mathrm{FIR}} / \mathrm{L}_{\mathrm{K}}\right)-(1.68 \pm 0.1)$ & 0.42 & $0.61 / 0.49$ & strong \\
\hline 17 & all & Var & $\mathrm{LOG}\left(\mathrm{M}_{\mathrm{X}}(\mathrm{gas}) /\left(\mathrm{M}_{\mathrm{H}_{2}}+\mathrm{M}_{\mathrm{HI}}\right)\right)=(0.33 \pm 0.16) \mathrm{LOG}\left(\mathrm{L}_{\mathrm{FIR}} / \mathrm{L}_{\mathrm{K}}\right)-(1.56 \pm 0.12)$ & 0.53 & $0.54 / 0.36$ & weak \\
\hline 17 & $>1 \frac{M_{\odot}}{y r}$ & Var & $\mathrm{LOG}\left(\mathrm{M}_{\mathrm{X}}(\right.$ gas $\left.) /\left(\mathrm{M}_{\mathrm{H}_{2}}+\mathrm{M}_{\mathrm{HI}}\right)\right)=(0.74 \pm 0.24) \mathrm{LOG}\left(\mathrm{L}_{\mathrm{FIR}} / \mathrm{L}_{\mathrm{K}}\right)-(1.49 \pm 0.12)$ & 0.51 & $0.65 / 0.54$ & strong \\
\hline
\end{tabular}

